\title{
Multiscale Modeling of Grain Boundary Segregation and Embrittlement in Tungsten for Mechanistic Design of Alloys for Coal Fired Plants
}

Type of Report: Final Scientific/Technical Report

Reporting Period Start Date: July 01, 2010

Reporting Period End Date: June 30, 2013

Principal Authors: Jian Luo, Vikas Tomar, Naixie Zhou, Hongsuk Lee

Date Report Was Issued: Septemeber 29, 2013

DoE Award Number: DE-FE0003892

Name of Recipient: Clemson University

Principal Investigators (PI's)*:

Original PI for 07/01/2010 -12/31/2013;

UCSD subcontractor for 01/01/2013-06/30/2013

Jian Luo, Ph.D.

Professor

Department of NanoEngineering

Program of Materials Science and Engineering

University of California, San Diego (UCSD)

Office: SME 244G

9500 Gilman Drive, Mail Code 0448

La Jolla, CA 92093

Phone: (858) 246-1203 Fax: (858) 534-9553

Email: jluo@ucsd.edu

\author{
“Managing" PI at Clemson for 01/01/2013 - 06/30/2013 \\ Fei Peng, Ph.D. \\ Assistant Professor \\ Department of Materials Science and Engineering \\ Program of Materials Science and Engineering \\ Clemson University, Clemson \\ Office: 210A Olin Hall \\ Clemson University \\ Clemson, SC 29634 \\ Phone: 864-656-4134 \\ Email: fpeng@clemson.edu
}

Co-PI \& Purdue Subcontractor:

Vikas Tomar, Ph.D.

Associated Professor

School of Aeronautics and Astronautics

Purdue University

701 W. Stadium Ave.

West Lafayette, IN 47907-2045

Phone: 765-494-3423

Email: tomar@purdue.edu

\footnotetext{
* The original PI, Dr. Jian Luo, has moved from Clemson to University of California, San Diego (UCSD) on January 1, 2013. DOE-NETL has approved to designate Dr. Fei Peng as a "managing PI" at Clemson for 01/01/2013 $06 / 30 / 2013$ and subcontracted the remaining funds at Clemson $(\$ 18,898)$ to UCSD.
} 


\section{DISCLAIMER}

This report was prepared as an account of work sponsored by an agency of the United States Government. Neither the United States Government nor any agency thereof, nor any of their employees, makes any warranty, express or implied, or assumes any legal liability or responsibility for the accuracy, completeness, or usefulness of any information, apparatus, product, or process disclosed, or represents that its use would not infringe privately owned rights. Reference herein to any specific commercial product, process, or service by trade name, trademark, manufacturer, or otherwise does not necessarily constitute or imply its endorsement, recommendation, or favoring by the United States Government or any agency thereof. The views and opinions of authors expressed herein do not necessarily state or reflect those of the United States Government or any agency thereof. 


\section{Abstract:}

Based on a recent discovery of premelting-like grain boundary segregation in refractory metals occurring at high temperatures and/or high alloying levels, this project investigated grain boundary segregation and embrittlement in tungsten (W) based alloys. Specifically, new interfacial thermodynamic models have been developed and quantified to predict high-temperature grain boundary segregation in the W-Ni binary alloy and W-Ni-Fe, W-Ni-Ti, W-Ni-Co, W-Ni-Cr, $\mathrm{W}-\mathrm{Ni}-\mathrm{Zr}$ and $\mathrm{W}-\mathrm{Ni}-\mathrm{Nb}$ ternary alloys. The thermodynamic modeling results have been experimentally validated for selected systems. Furthermore, multiscale modeling has been conducted at continuum, atomistic and quantum-mechanical levels to link grain boundary segregation with embrittlement. In summary, this 3-year project has successfully developed a theoretical framework in combination with a multiscale modeling strategy for predicting grain boundary segregation and embrittlement in $\mathrm{W}$ based alloys. 


\section{TABLE OF CONTENTS}

$\begin{array}{ll}\text { Abstract } & 3\end{array}$

$\begin{array}{ll}\text { I. Executive Summary } & 5\end{array}$

II. Report Details $\quad 6$

Experimental Methods (This is mostly a modeling project. Experimental results were reported only in $\S 3$. Relevant experimental procedures are described on pages 28-29)

$\begin{array}{ll}\text { Results and Discussions } & 6\end{array}$

§1. Model Derivation $\quad 6$

§2. Systematic Numerical Calculation of W-Ni-X $\lambda$ Diagrams 11

§3. Experiments Validation of Thermodynamic Theories/Modeling Results 28

$\S 4$. Investigation of Interfacial Property of Ni-doped W Using Quantum Simulation

§5. Investigation on the Role of Grain Boundary in the Fracture using 3-D

Extended Finite Element Method

§6. Simulation of Multiscale Model of Grain Boundary in The Fracture Using Cohesive Finite Element Method $\quad 65$

$\begin{array}{ll}\text { Conclusions } & 69\end{array}$

$\begin{array}{ll}\text { III. Graphical Materials List } & 70\end{array}$

$\begin{array}{ll}\text { IV. References } & 74\end{array}$

V. List of Acronyms and Abbreviations $\quad 76$

$\begin{array}{ll}\text { VI. Appendices } & 77\end{array}$

(A) Personnel $\quad 77$

$\begin{array}{ll}\text { (B) Cost Status } & 77\end{array}$

$\begin{array}{ll}\text { (C) Milestone Status } & 78\end{array}$

$\begin{array}{ll}\text { (D) List of Publications in Refereed Journals } & 78\end{array}$ 


\section{Executive Summary}

Based on a recent discovery of premelting-like grain boundary (GB) segregation in refractory metals occurring at high temperatures and/or high alloying levels by Luo's group at Clemson, this project investigated GB segregation and embrittlement in tungsten (W) based alloys. This project has successfully developed a theoretical framework in combination with a multiscale modeling strategy for predicting GB segregation and embrittlement in W based alloys.

Specifically, the following tasks have been successfully completed: 1) a new high-temperature GB segregation theory/model has been developed for W-based ternary alloys; 2) a systematic numerical experiment has been conducted for $\mathrm{W}-\mathrm{Ni}-\mathrm{X}$ alloys (where $\mathrm{X}$ is a fictive element) to identify the key thermodynamic factors that control GB segregation behaviors in W-based ternary alloys; 3) six real ternary alloys (W-Ni-Fe, W-Ni-Ti, W-Ni-Co, W-Ni-Cr, W-Ni-Zr and W-Ni-Nb) have been modeled; 4) controlled experiments has been conducted to validate the thermodynamic theory and modeling results using a series of ternary $\mathrm{W}-0.5 \mathrm{Ni}-0.5 \mathrm{X}$ at. \% (where $\mathrm{X}=\mathrm{Cr}, \mathrm{Zr}, \mathrm{Co}$, $\mathrm{Fe}, \mathrm{Nb}, \mathrm{Ti}$ ) alloys; 5) quantum simulation has been conducted to investigate the interfacial property of Ni-doped $\mathrm{W}$; and 6) a 3-D extended finite element method has been employed to investigate the role of GB in the fracture at continuum level.

In summary, this project has developed thermodynamic theories to predict high-temperature GB segregation in binary and ternary $\mathrm{W}$ alloys, experimentally validated the developed thermodynamic theories and modeling results on selected systems, and conducted multiscale modeling (at continuum, atomistic and quantum-mechanical levels) to link GB segregation with GB embrittlement. The project has achieved two key milestones as planned. First, a thermodynamic description for high-temperature premelting-like GB segregation in multicomponent refractory alloys has been developed (by 9/30/2011). Second, multiscale modeling strategies has been developed to link grain boundary segregation with embrittlement (by 6/30/2013). 


\section{Report Details}

\section{Results and Discussions}

\section{Part A (§1-§3): Thermodynamic Modeling and Experimental Validation}

\section{§1. Model Derivation}

\subsection{Model Formulation and Numerical Methods}

In prior studies, we extended bulk CALPHAD (Calculation of Phase Diagram) methods to compute "GB diagrams" for tenary (A-B-C) alloys. The basic feasibility for computing such GB diagram has been demonstrated for binary refractory alloys by the PI's group ${ }^{1,2}$ and the correctness of this model and computation results has been verified by direct high-resolution transmission electron microscopy (HRTEM) and Auger electron spectroscopy (AES) measurements as well as indirect probing of GB diffusion via designed sintering experiments ${ }^{3-5}$.

In this project, we aim to extend this theory to model tenary alloys. To achieve this, we generalized a Miedema-type statistical model to compute the grain-boundary energy $\gamma_{\mathrm{GB}}$ for a ternary alloy:

$$
\gamma_{G B}^{(0)}=\sum_{i=A, B, C} F_{i}^{\text {solid }} \gamma_{G B(i)}
$$

In the above equation, $F_{i}^{\text {solid }}$ is the "surface fraction" of the $i^{\text {th }}$ component on a grain surface and $\gamma_{G B(i)}$ is the GB energy $\left[\gamma_{G B(i)} \approx \Delta H_{i}^{\text {vap }} /\left(3 C_{0} V_{i}^{2 / 3}\right)\right]$, where $\Delta H_{i}^{\text {vap }}$ is the vaporization enthalpy and $V_{i}$ is the atomic volume. On the other hand, we also proposed a model to compute crystal-liquid interfacial energy $\gamma_{\mathrm{cl}}$ for a multicomponent alloy:

$$
\gamma_{c l}=\sum_{j=A, B, C} F_{j}^{\text {solid }} \times\left(\frac{\Delta H_{j}^{\text {fuse }}}{C_{0} V_{j}^{2 / 3}}+\sum_{i \neq j} \frac{\Delta H_{\{j \text { in } i\}}^{\text {interface }} F_{i}^{\text {liquid }}}{C_{0} V_{j}^{2 / 3}}\right)+\frac{1.9 R T}{C_{0} V_{\text {Average }}^{2 / 3}}-\gamma^{\text {ref }}
$$

In the above equation, $\Delta H^{\mathrm{fuse}}$ is fusion enthalpy, and the subscript " $i$ " and " $j$ " denote the element $i$ and $j . \Delta H_{\{j \text { in } i\}}^{\text {interfec }}$ are the enthalpy of solution, where the curly and angle braces denote liquid phase and solid phase respectively. $F_{i}^{\text {liquid }}$ is the "surface fraction" of $i$ atoms at the liquid side of liquid-crystal interface. $1.9 R T / C_{0} V_{\text {Average }}^{2 / 3}$ is the entropic contribution due to local ordering. $\gamma^{\text {ref }}$ is the reference energy state of the interface. In our case, the reference energy is given by:

$$
\gamma^{r e f}=\sum_{i \neq j} \frac{\Delta H_{\{j \text { in } i\}}^{\text {interface }} F_{i}^{\text {liquid }} F_{j}^{\text {liquid }}}{2 C_{0} V_{j}^{2 / 3}}-\sum_{i \neq j} \frac{\Delta H_{\langle j \text { in } i\rangle}^{\text {interace }} F_{i}^{\text {solid }} F_{j}^{\text {solid }}}{2 C_{0} V_{j}^{2 / 3}}
$$

Furthermore, we adopt multicomponent bulk CALPHAD methods to compute the free energy penalty to form an undercooled liquid in the A-B-C system, as

$$
\Delta G_{\mathrm{amorph}}=G_{\text {liq }}^{f}-\left(X_{\mathrm{A}}^{\mathrm{liq}} \mu_{\mathrm{A}}+X_{\mathrm{B}}^{\text {liq }} \mu_{\mathrm{B}}+X_{\mathrm{C}}^{\text {liq }} \mu_{\mathrm{C}}\right),
$$

where the Redlich-Kister-Muggianu procedure is used to extrapolate the formation free energy expression of the related liquid and solid phases. 


$$
\begin{aligned}
G^{f}\left(T, X_{A}, X_{B}\right)= & X_{A} G_{A}^{0}+X_{B} G_{B}^{0}+X_{C} G_{C}^{0}+R T\left(X_{A} \ln X_{A}+X_{B} \ln X_{B}+X_{C} \ln X_{C}\right)+X_{A} X_{B} \sum_{j=0}^{n_{A B}} \Omega_{j}^{A B}\left(X_{A}-X_{B}\right)^{j} \\
& +X_{B} X_{C} \sum_{j=0}^{n_{B} C} \Omega_{j}^{B C}\left(X_{B}-X_{C}\right)^{j}+X_{C} X_{A} \sum_{j=0}^{n_{C A}} \Omega_{j}^{C A}\left(X_{C}-X_{A}\right)^{j}+X_{A} X_{B} X_{C} G^{A B C}
\end{aligned}
$$

Finally, we compute $\lambda \equiv \max \left(\Delta \gamma / \Delta G_{\text {amorph }}\right)$, which is the maximum thickness of a stable quasi-liquid intergranular film (IGF) without the consideration of interfacial forces. The computed $\lambda$ value represent the thermodynamic tendency for general GBs to disorder (at fixed bulk chemical potentials and temperature). By computing $\lambda$ as a function of composition and temperature, we obtained a GB " $\lambda$ diagram."

A MATLAB code was developed and applied it to calculate the GB " $\lambda$ diagram" of $(\mathrm{Ni}+\mathrm{Cr})$ co-doped $\mathrm{W}$ numerically. The W-Ni-Cr GB phase diagrams were calculated for a selected composition area (0-0.05\% Ni; 0-0.7\% Cr) which exhibit a single BCC phase (i.e., a W based solid solution), and two isothermal sections were shown in Fig. 1. Fig.1 illustrates that the computed $\lambda$ value (which indicates the adsorption and disordering levels) decreases with increasing temperature. This counterintuitive behavior has recently been verified by the PI's group $^{6}$. The diagram also showed that $\mathrm{Cr}$ doping to the $\mathrm{W}-\mathrm{Ni}$ system will reduces the GB segregation.
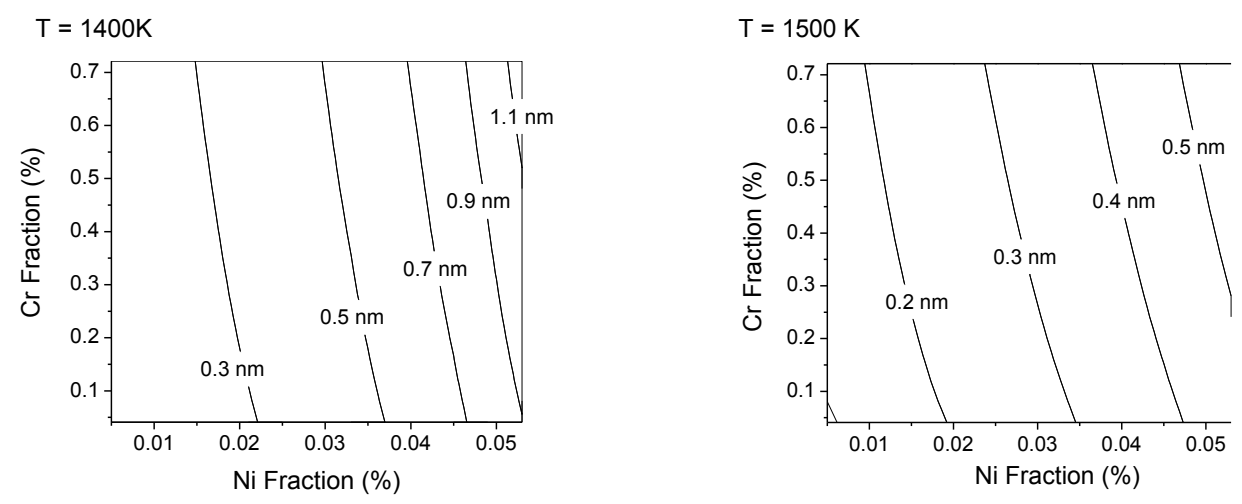

Figure 1: Computed isothermal sections of a computed "GB diagram" for $(\mathrm{Ni}+\mathrm{Cr})$ co-doped $\mathrm{W}$ at (left) $1400 \mathrm{~K}$ and (right) $1500 \mathrm{~K}$, respectively. Contour plots of constant $\lambda$ values are shown. The computed $\lambda$ value corresponds to the degree of disorder at GBs, which is coupled with the adsorption level.

In order to plot a $\lambda$ diagram onto the bulk phase diagram, we have developed a MATLAB code to compute the bulk "tie lines" for the equilibrium solid and liquid phases in a ternary system. When two phases ( $s$ and $l$ ) are in equilibrium in a ternary system, the following three equations should be satisfied: 


$$
\begin{aligned}
& \Delta G_{s}\left(X^{s o l}\right)-\Delta G_{l}\left(X^{l i q}\right)+\mu_{s}^{B}\left(X_{B}^{s o l}-X_{B}^{l i q}\right)+\mu_{s}^{C}\left(X_{C}^{s o l}-X_{C}^{l i q}\right)=0 \\
& \mu_{s}^{B}-\mu_{l}^{B}=0 \\
& \mu_{s}^{C}-\mu_{l}^{C}=0
\end{aligned}
$$

where $X_{B}, X_{C}$ are the two alloy components, $X^{<\Phi>}$ is the composition vector of $\Phi$ phase; $\mu$ and $G$ represents the chemical potential and free energy of a specific phase. The following Newton iteration method is used to solve the above nonlinear equations:

$$
X^{k+1}=X^{k}-\left[\frac{\partial f\left(X^{k}\right)}{\partial X}\right]^{-1} \partial f\left(X^{k}\right) \quad(k=0,1, \cdots),
$$

where $x$ is the variable vector and $f$ is the target function. The derivative term is the Jacobian matrix, which can be calculated numerically from:

$$
\left[A\left(X^{k}, h^{k}\right)\right]=\frac{f_{i}\left(X^{k}+h_{j}^{k} e_{j}\right)-f_{i}\left(X^{k}\right)}{h_{j}^{k}} \quad(i, j=1,2, \cdots, n) .
$$

Fig. 2 illustrates an isothermal section of a GB diagram for $(\mathrm{Ni}+\mathrm{Cr})$ co-doped $\mathrm{W}$. Noting that the contour lines are almost parallel to the solidus line when it is close to the solubility limit.

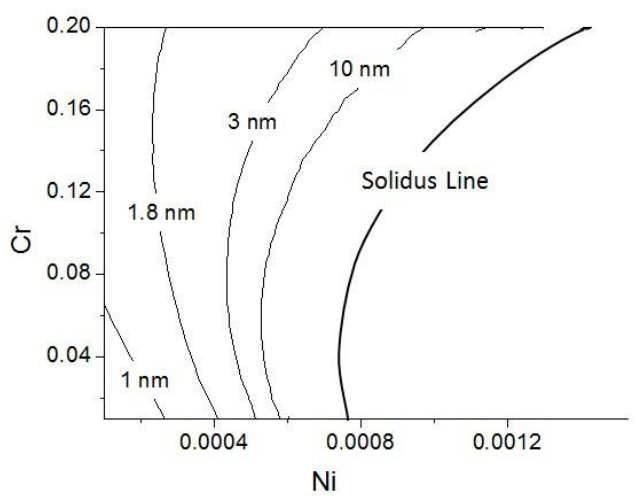

Figure 2: A computed isothermal section of a GB "phase" diagram for $(\mathrm{Ni}+\mathrm{Cr})$ co-doped $\mathrm{W}$ at $1600 \mathrm{~K}$. The contour plots of constant $\lambda$ values are shown. The computed solidus line is also shown.

\section{2 (Approximated) Analytical Approaches}

In a parallel thrust, we also derived analytical approaches to estimate the thickness of a liquid-like intergranular film (IGF) to better elucidate the underlying physics. In a prior work of the PI and a coworker ${ }^{5}$, an analytical method was developed to estimate IGF thickness in binary alloys assuming the solid phases can be represented by regular solutions. In this project, we further extend this method to tenary alloys. In a ternary alloy, we assume that the IGF composition takes on the liquidus compositions $\left(X_{l}, Y_{l}\right)$, and $\left(X_{S}, Y_{S}\right)$ represent the corresponding solidus compositions. For an alloy in the single-phase region with the bulk composition of $(X, Y)$, the 
free-energy penalty for forming an undercooled liquid is given by:

$$
\Delta G_{\text {amorph }} \approx \Delta G_{s}+\Delta \mu_{X}\left(X_{l}-X_{s}\right)+\Delta \mu_{Y}\left(Y_{l}-Y_{S}\right)-\left[\left.\frac{\partial G_{s}}{\partial X}\right|_{X, Y} \Delta X+\left.\frac{\partial G_{s}}{\partial Y}\right|_{X, Y} \Delta Y\right]
$$

where

$$
\begin{aligned}
& \Delta G=G_{s}\left(X_{s}, Y_{s}\right)-G_{s}(X, Y) ; \\
& \Delta \mu_{X}=\left.\frac{\partial G_{s}}{\partial X}\right|_{\left(X_{s}, Y_{s}\right)}-\left.\frac{\partial G_{s}}{\partial X}\right|_{X, Y}, \Delta \mu_{Y}=\left.\frac{\partial G_{s}}{\partial Y}\right|_{\left(X_{s}, Y_{s}\right)}-\left.\frac{\partial G_{s}}{\partial Y}\right|_{X, Y} \text { and } \\
& \Delta X=X_{s}-X, \Delta Y=Y_{s}-Y .
\end{aligned}
$$

Using the Taylor expansion, $\Delta G_{\mathrm{s}}$ and $\Delta \mu_{\mathrm{x}}$ at $\left(X_{\mathrm{s}}, Y_{\mathrm{s}}\right)$ Eq. (9) could be written as:

$$
\begin{aligned}
& \left.\Delta G_{\text {amoph }} \approx \Delta X\left(X_{l}-X_{s}\right) \frac{\partial^{2} G_{s}}{\partial X^{2}}\right|_{X_{s}, Y_{s}}+\left.\left[\Delta Y\left(X_{l}-X_{s}\right)+\Delta X\left(Y_{l}-Y_{s}\right)\right] \frac{\partial^{2} G_{s}}{\partial Y \partial X}\right|_{X_{s}, Y_{s}}+\left.\Delta Y\left(Y_{l}-Y_{s}\right) \frac{\partial^{2} G_{s}}{\partial Y^{2}}\right|_{X_{s}, Y_{s}}+\ldots \\
& =\left[\begin{array}{ll}
\Delta X & \Delta Y
\end{array}\right]\left[\begin{array}{ll}
\frac{\partial^{2} G_{s}}{\partial X^{2}} & \frac{\partial^{2} G_{s}}{\partial Y \partial X} \\
\frac{\partial^{2} G_{s}}{\partial Y \partial X} & \frac{\partial^{2} G_{s}}{\partial Y^{2}}
\end{array}\right]_{X_{s}, Y_{s}}\left[\begin{array}{l}
\left(X_{l}-X_{s}\right) \\
\left(Y_{l}-Y_{s}\right)
\end{array}\right]
\end{aligned}
$$

For a binary system, Eq. (10) can be simplified to:

$$
\left.\Delta G_{\text {amorph }} \approx\left(X_{l}-X_{s}\right) \frac{d^{2} G_{s}}{d X^{2}}\right|_{X_{s}} \Delta X+R(\Delta X)
$$

If the solid phase can be treated as a regular solution with $\Omega$ being the regular solution parameter, we can obtain the following approximation:

$$
\Delta G_{\text {amorph }}^{\left(\text {(1) }^{\text {st }} \text { order }\right)} \approx\left(X_{l}-X_{s}\right)\left[R T\left(\frac{1}{X_{s}}+\frac{1}{1-X_{s}}\right)-2 \Omega\right] \Delta X
$$

An approximated analytical solution for a binary alloy derived in our prior work ${ }^{5}$ is given by:

$$
\Delta G_{\text {amorph }}^{\text {(exact) }} \approx R T\left[X_{l} \ln \frac{X_{s}}{X_{s}-\Delta X}+\left(1-X_{l}\right) \ln \frac{1-X_{s}}{1-X_{s}+\Delta X}\right]-\Omega \Delta X\left(2 X_{l}-2 X_{s}+\Delta X\right)
$$

A comparison of results computed by Eq. (12), Eq. (13) and second order expansion in Mo-Ni system is given in Fig. 3. Further, we are developing methods to incorporate both liquid and solid phases' thermodynamic properties into our analysis. 


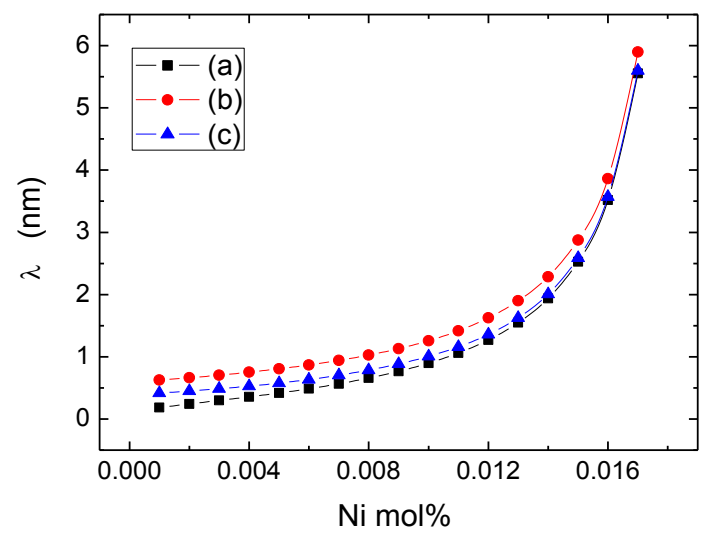

Figure 3: Comparison of the estimated $\lambda$ values for Ni-doped Mo at $1400 \mathrm{~K}$ using different (approximated) analytical solutions according to: (a) Eq. (11) ref [4]; (b) Eq. (10) as a first-order approximation; and (c) a second-order approximation of Eq. (8) using the Taylor expansion.

The validity of the Eq. (10) for ternary systems was further verified by comparing with numerical results for W-Ni-Cr: see Fig. 2 vs. Fig. 4. In the next stage, we will use both numerical and analytical approaches to identify the key thermodynamic factors that control the high-T adsorption and disordering in ternary alloys.

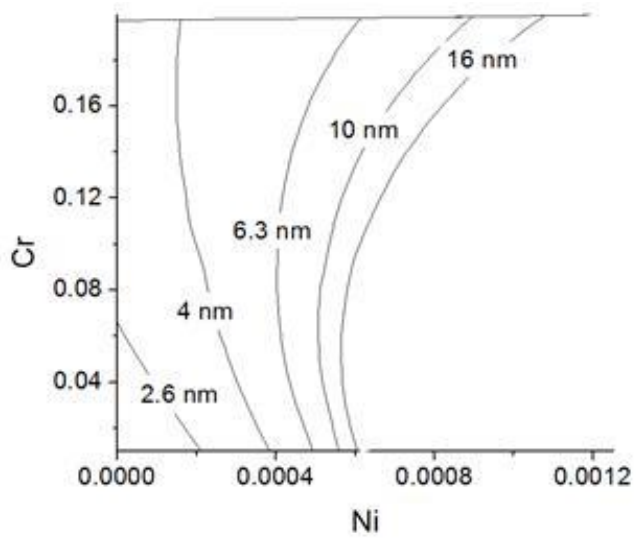

Figure 4: A computed $\lambda$ diagram using an approximate analytical solution [Eq. (10)] for ( $\mathrm{Ni}+$ $\mathrm{Cr}$ ) co-doped $\mathrm{W}$ at $1400 \mathrm{~K}$. The results are generally consistent the "exact" solution computed by numerical methods shown in Fig. 2.

\subsection{Film Compositions Prediction}

In order to achieve deeper understanding of IGF, we further obtained the "optimal" film composition $\left(X_{\text {film }}\right)$ values that will minimize the excess free energies under the assumption of uniform film composition (no gradients). An example is shown in Fig. 5 (a). 


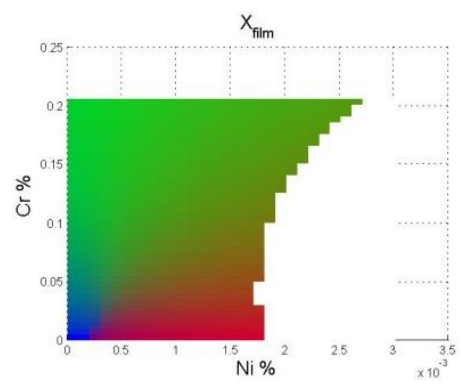

(a)

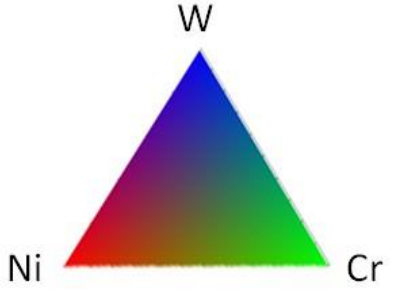

(b)

Figure 5: (a) The $\mathrm{X}_{\text {film }}$ map of $\mathrm{W}-\mathrm{Ni}-\mathrm{Cr}$ system.

\section{§2. Systematic Numerical Calculation of W-Ni-X $\lambda$ Diagrams}

\subsection{Modeling of W-Ni- $X \wedge$ Diagrams ( $X$ is artificial element)}

Firstly, we conducted a systematic numerical experiment to compute GB diagrams for W-Ni- $X$ system, where $X$ is an "artificial" (fictive) metal-like element with adjustable physical properties. By doing this, we hope to understand what are the key thermodynamic parameters that control the GB behaviors.

We first identify the parameters that control the properties of $X$ and how it interacts with $\mathrm{W}$ and Ni. These parameters can be classified into three groups: 1) perperties of $X$, including the free energies of the liquid phase $\left({ }^{\circ} \mathrm{G}_{\mathrm{X}}{ }^{\text {(liquid) }}\right)$ and the solid phase $\left({ }^{\circ} \mathrm{G}_{\mathrm{X}}{ }^{\text {(liquid) }}\right)$, fusion enthalpy $\left(\Delta H_{\mathrm{X}}{ }^{\text {fuse }}\right)$, metlting temperature $\left(T_{\mathrm{m}-\mathrm{X}}\right)$ and vaporization enthalpy $\left.\left(\Delta H_{\mathrm{X}}{ }^{\mathrm{vap}}\right) ; 2\right)$ interaction parameters, such as the regular solution parameter $\left(\Omega_{\mathrm{X}-\mathrm{I}}\right)$ and enthalpy of solution $\left(\Delta H^{\text {interface }}\right)$; and $\left.\mathbf{3}\right)$ other parameters, such as the atomic volume $\left(V_{\mathrm{X}}\right)$. These parameters are dependent on each other.

We adopt the following approxmations for the first group of parameters (based on an assessments made for transition metals that are shown in Fig. 6). First, fusion enthalpy is scaled by melting temperature (with a constant fusion entropy of $10 \mathrm{~J} / \mathrm{mol} / \mathrm{K}$ ):

$$
\Delta H_{\mathrm{X}}^{\text {fuse }} \approx 10 T_{\mathrm{m}}(\mathrm{J} / \mathrm{mol})
$$

Second, the atomization and fusion enthalpies are linearly correlated (i.e., a solid-to-liquid transition breaks approximately $4 \%$ of bonds; $\Delta H_{\mathrm{X}}{ }^{\text {fuse }} \approx 0.04 \Delta H_{\mathrm{X}}{ }^{\text {vap }}$ ) so that:

$$
\Delta H_{\mathrm{X}}{ }^{\mathrm{vap}} \approx 10 T_{\mathrm{m}} / 0.04(\mathrm{~J} / \mathrm{mol})
$$

Third, we set the BCC phase of pure $X$ as the referencing state (with zero free energy) and omit the heat capacity change upon melting. Then, ${ }^{\circ} \mathrm{G}_{\mathrm{X}}{ }^{\text {(liquid) }}$ could be expressed as:

$$
{ }^{\circ} \mathrm{G}_{\mathrm{X}}{ }^{\text {(liquid) }} \approx 10\left(\mathrm{~T}_{\mathrm{m}}-\mathrm{T}\right)
$$

Finally, $\underline{\mathrm{V}}_{\mathrm{X}}$ is set to $8 \mathrm{~cm}^{3} / \mathrm{mol}$, which is the average molar volume of the transition metals. 
a)

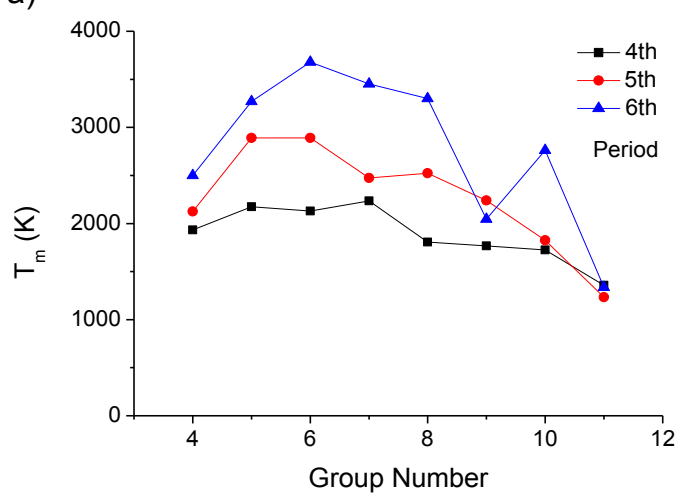

c)

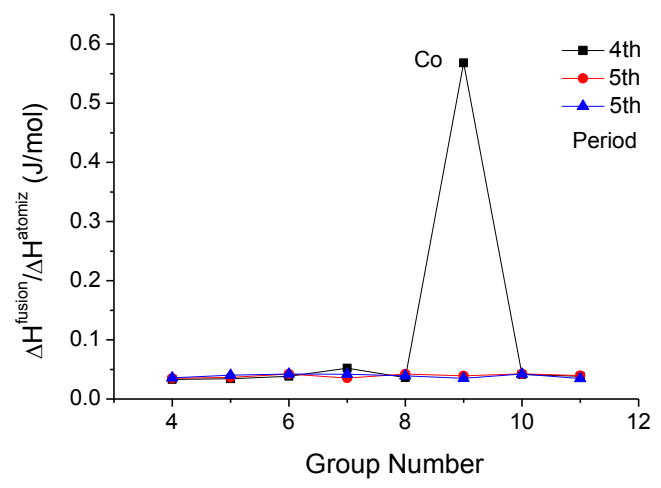

b)

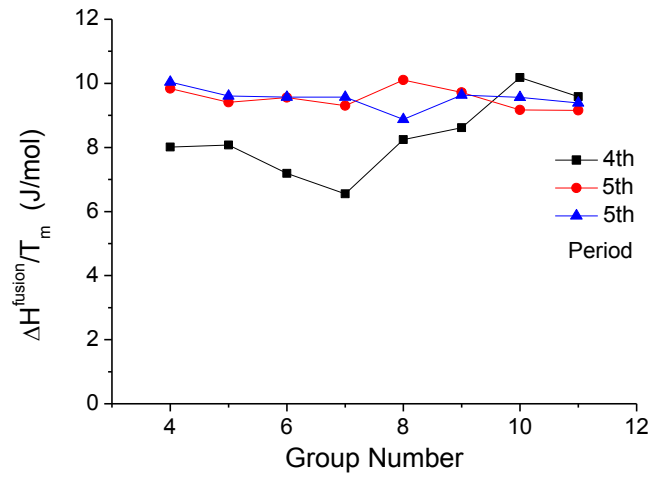

d)

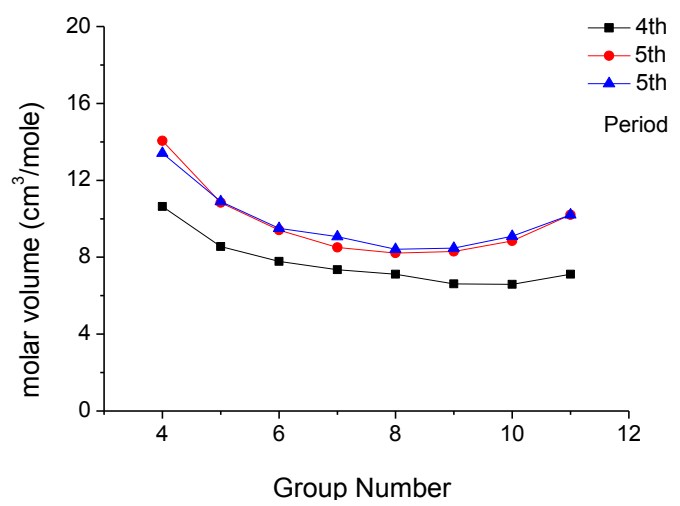

Figure 6: The physical properties of transition metals.

We further adopt the regular solution model to describe the binary interaction with the regular-solution parameter $\Omega$. Table I lists some typical regular-solution parameters. For $\mathrm{W}-X$, $\Omega^{\text {liquid }}$ is usually $\sim 50 \mathrm{~kJ} / \mathrm{mol}$ lower than $\Omega^{\text {solid }}$. Additionally, $\mathrm{W}$ generally is harder to mix with other elements than Ni in both solid (BCC) and liquid phase, as represented by the generally larger $\Omega$ 's.

Table I: Regular solution parameters ( $\Omega$ ’s) from the SGTE binary database.

\begin{tabular}{|c|c|c|c|}
\hline$X=$ & Ni- $X$ (Solid) $(\mathrm{kJ} / \mathrm{mol})$ & $X=$ & Ni- $X$ (Liquid) $(\mathrm{kJ} / \mathrm{mol})$ \\
\hline $\mathrm{Ta}$ & -55.77 & $\mathrm{Ta}$ & -105.15 \\
\hline $\mathrm{Nb}$ & -13.7 & $\mathrm{Nb}$ & -89 \\
\hline $\mathrm{Ti}$ & -85.31 & $\mathrm{Ti}$ & -153.31 \\
\hline $\mathrm{W}$ & $\mathbf{8 6 . 0 4}$ & $\mathrm{W}$ & 14 \\
\hline & $\mathrm{W}-\mathrm{X}$ (Solid) $(\mathrm{kJ} / \mathrm{mol})$ & & $\mathrm{W}-\mathrm{X}$ (Liquid) $(\mathrm{kJ} / \mathrm{mol})$ \\
\hline $\mathrm{Co}$ & $\mathbf{5 4 . 6 5}$ & $\mathrm{Co}$ & $\mathbf{0}$ \\
\hline $\mathrm{Cr}$ & 31.52 & $\mathrm{Cr}$ & 4.5 \\
\hline $\mathrm{Fe}$ & 41.54 & $\mathrm{Fe}$ & 0 \\
\hline $\mathrm{Mo}$ & 2.00 & $\mathrm{Mo}$ & 0 \\
\hline $\mathrm{Ti}$ & 17 & $\mathrm{Ti}$ & 10 \\
\hline
\end{tabular}


In the second group of parameters, the enthalpy of solution $\left(\Delta H^{\text {interface }}\right)$ given by the Miedema model can be related to the regular-solution parameter $(\Omega)$. If we ignore the difference in molar volume, we can derive the following relation:

$$
\Delta H_{X \text { in } i}^{\text {interfec }} \approx \Delta H_{i \text { in } X}^{\text {interface }} \approx \Omega_{X-i}^{\text {liquid }} .
$$

In summary, using the approximations and simplifications given by Eqs. (15)-(18) and ignoring the ternary interaction, we can represent the $\mathrm{W}-\mathrm{Ni}-X$ system by five independent parameters:

$$
T_{\mathrm{m}}^{\mathrm{X}}, \Omega_{W-X}^{\text {liquid }}, \Omega_{W-X}^{b c c}, \Omega_{N i-X}^{\text {liquid }}, \text { and } \Omega_{N i-X}^{b c c} .
$$

\subsection{Numeric Simulations}

Numerical Experiment (A): X mixes ideally with Ni in both solid and liquid phases

In the first numerical experiment, we modeled the $\mathrm{W}-\mathrm{Ni}-X$ system where $X$ forms ideal solutions with $\mathrm{W}$ and $\mathrm{Ni}$ in both solid and liquid phases (i.e., $\Omega_{W-X}^{\text {liquid }}=\Omega_{W-X}^{b c c}=\Omega_{N i-X}^{\text {liquid }},=\Omega_{N i-X}^{b c c}=0$ ). Thus, we only have one variable, $T_{\mathrm{m}}{ }^{\mathrm{X}}$, and we varied it from $1000 \mathrm{~K}$ to $3000 \mathrm{~K}$. Several representative computed GB $\lambda$ diagrams for (Ni-X) co-doped $\mathrm{W}$ at $T=1673 \mathrm{~K}$ (with different $T_{\mathrm{m}}{ }^{\mathrm{X}}$ ) are shown in Fig. 7, which illustrates that the addition of $X$ will intend to dry/crystallize GBs in (Ni-X) co-doped $\mathrm{W}$ if the melting point of $\mathrm{X}$ is high. The corresponding $X_{\text {film }}$ maps are shown in Fig. 8.
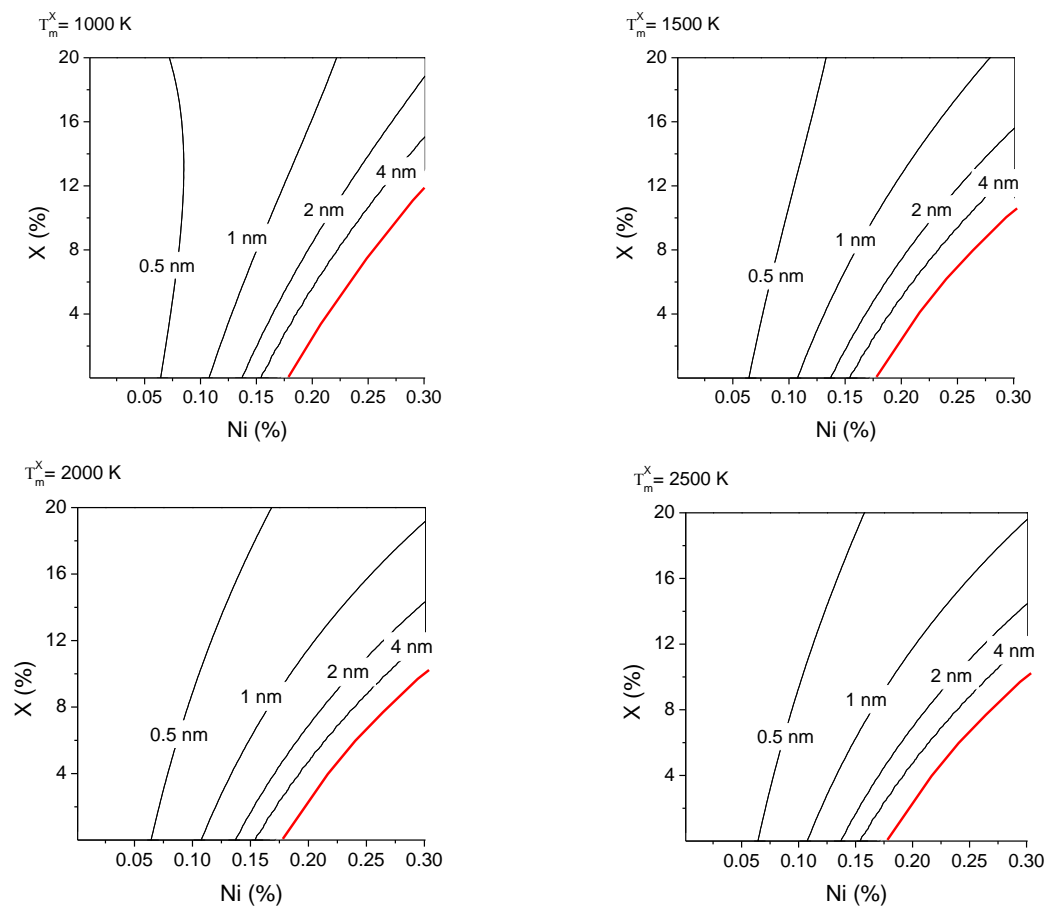

Figure 7: Computed $\lambda$ diagrams for the W-Ni-X system assuming: $\Omega_{W-X}^{\text {liquid }}=\Omega_{W-X}^{b c c}=\Omega_{N i-X}^{\text {liquid }}=$ $\Omega_{N i-X}^{b c c}=0$, and $\mathrm{T}=1673 \mathrm{~K}$. The values of $T_{\mathrm{m}}{ }^{\mathrm{X}}$ are labeled. Red lines are the solidus lines. 

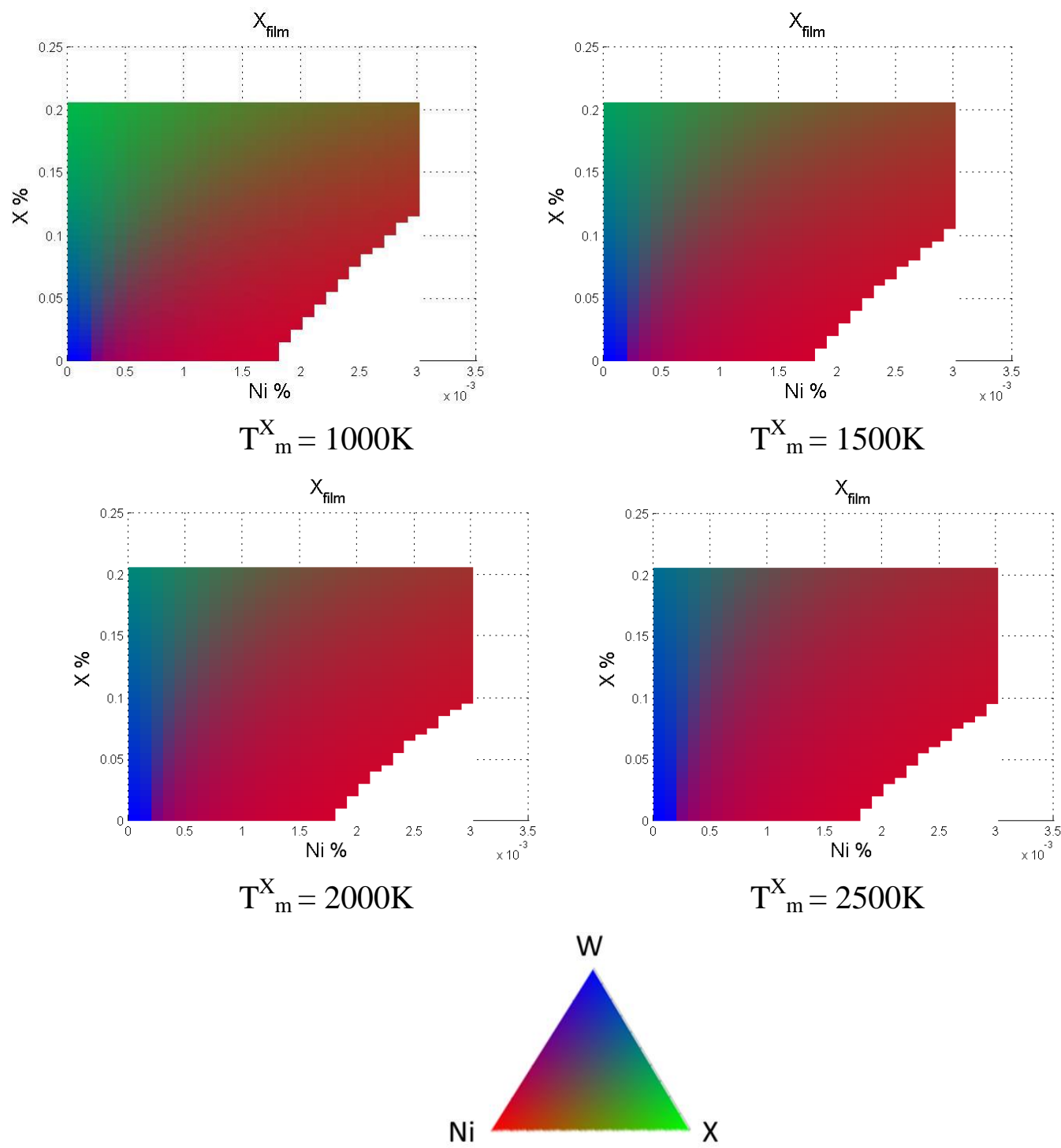

$X_{\text {film }}$ Color Map

Figure 8: Computed $X_{\text {film }}$ maps for the W-Ni-X system assuming: $\Omega_{W-X}^{\text {liquid }}=\Omega_{W-X}^{b c c}=\Omega_{N i-X}^{\text {liquid }}=$ $\Omega_{N i-X}^{b c c}=0$, and $\mathrm{T}=1673 \mathrm{~K}$. 
Numerical Experiment (B): X forms a regular solution with W in the bcc phase

In our second experiment, $X$ is set to form ideal solution with $\mathrm{W}$ in liquid phase (i.e., $\Omega_{W-X}^{\text {liquid }}=$ $\left.\Omega_{N i-X}^{\text {liquid }},=\Omega_{N i-X}^{b c c}=0\right)$. We set $T_{\mathrm{m}}{ }^{\mathrm{X}}=2000 \mathrm{~K}$. Only $\Omega_{W-X}^{b c c}$ is set to be a variable, which is varied from $-50 \mathrm{KJ} / \mathrm{mol}$ to $50 \mathrm{KJ} / \mathrm{mol}$. Several representative computed GB $\lambda$ diagrams for (Ni-X) co-doped $\mathrm{W}$ at $T=1673 \mathrm{~K}$ were illustrated in Fig. 9, which shows that the addition of $\mathrm{X}$ will promote GB disorder in (Ni-X) co-doped $\mathrm{W}$ for a large positive $\Omega_{W-X}^{b c c}$. The corresponding $X_{\text {film }}$ maps are shown in Fig. 10. In general, a more positive $\Omega_{W-X}^{b c c}$ will make X easier to be absorbed at W GBs.
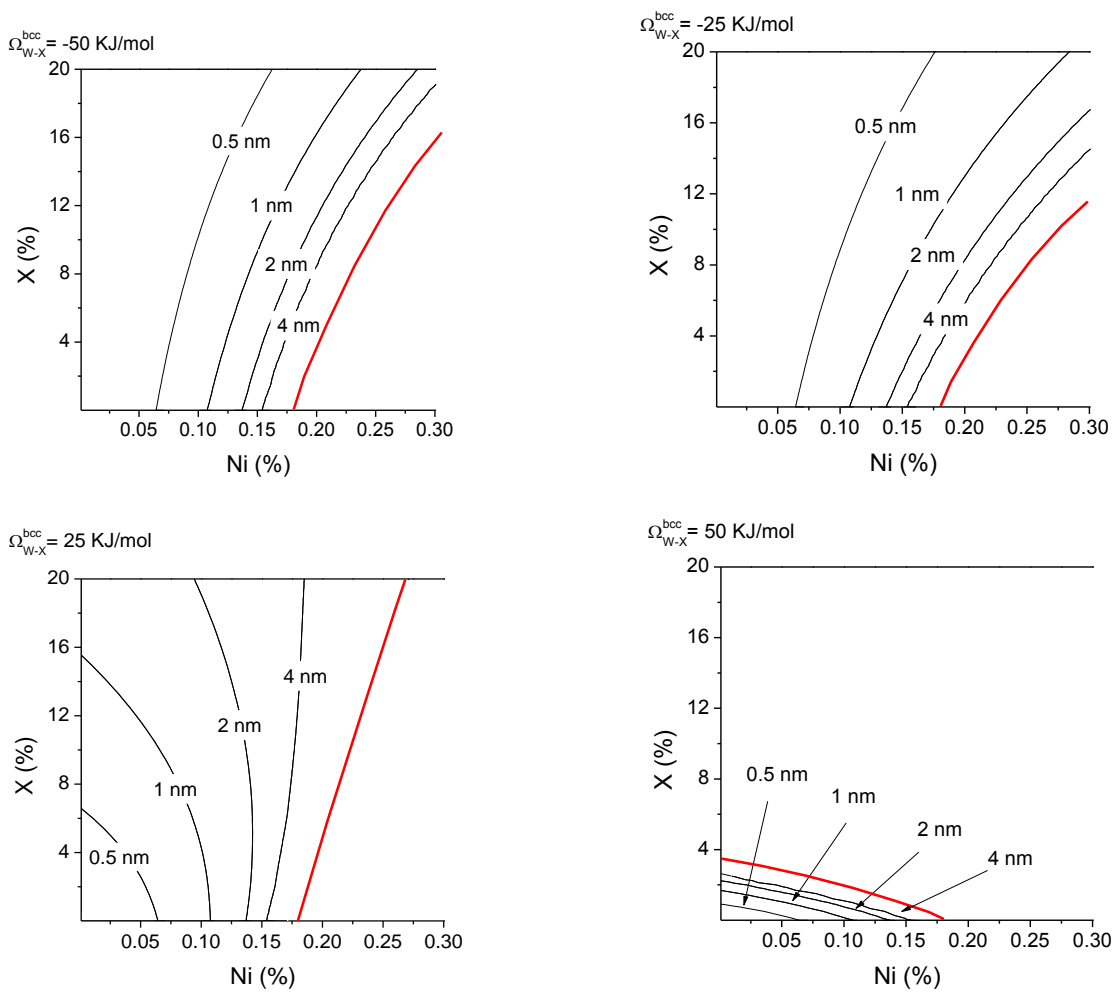

Figure 9: Computed GB ( $\lambda$ ) diagrams for the W-Ni-X system for: $\Omega_{W-X}^{\text {liquid }}=\Omega_{N i-X}^{\text {liquid }}=\Omega_{N i-X}^{b c c}=0, \mathrm{~T}=$ $1673 \mathrm{~K}$ and $T_{\mathrm{m}}{ }^{\mathrm{X}}=2000 \mathrm{~K}$. The values of $\Omega_{W-X}^{b c c}$ are labeled. 

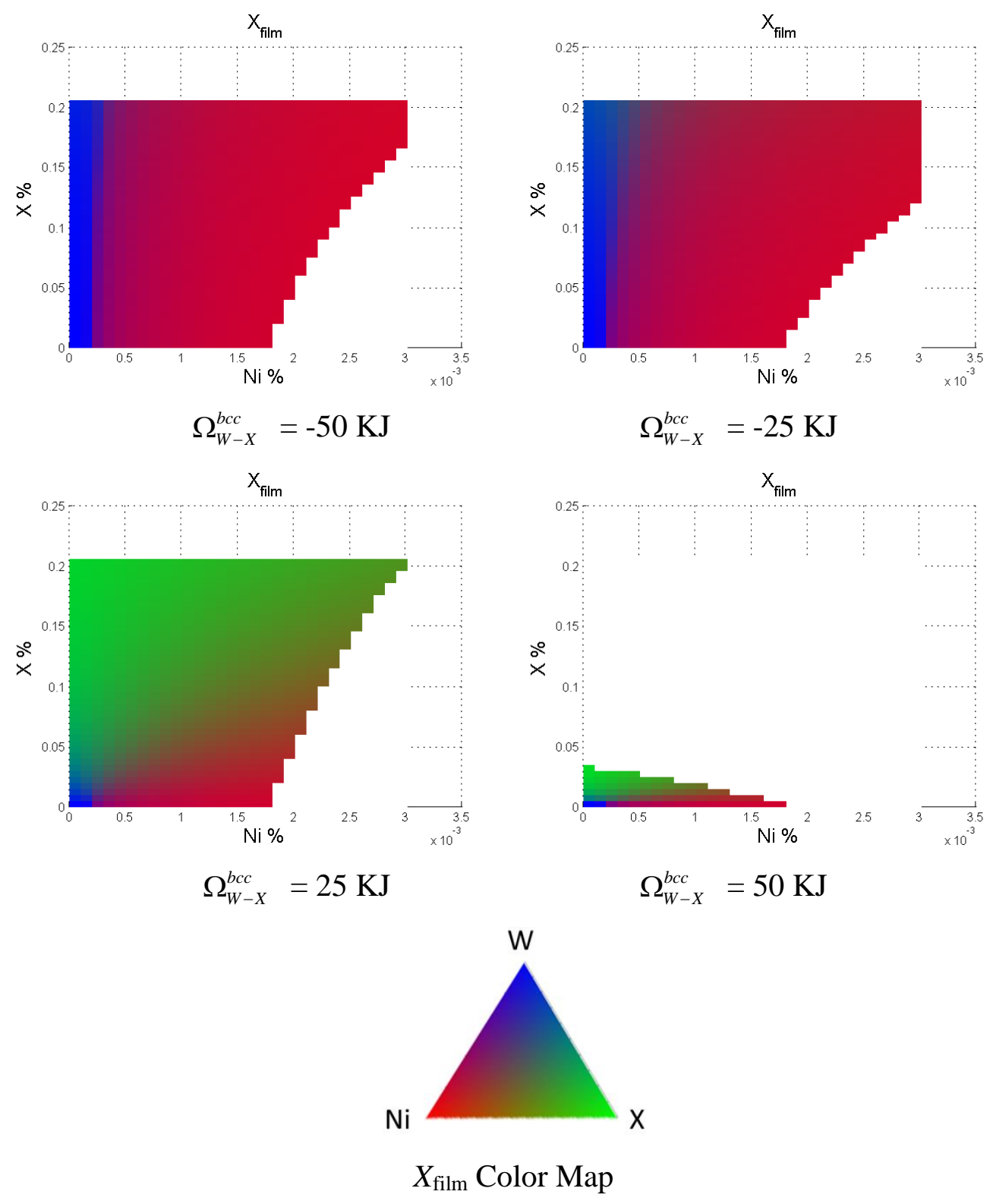

Figure 10: Computed $X_{\text {film }}$ maps for the W-Ni-X system assuming: $\Omega_{W-X}^{\text {liquid }}=\Omega_{N i-X}^{\text {liquid }}=\Omega_{N i-X}^{b c c}=0, \mathrm{~T}$ $=1673 \mathrm{~K}$ and $T_{\mathrm{m}}{ }^{\mathrm{X}}=2000 \mathrm{~K}$. The values of $\Omega_{W-X}^{b c c}$ are labeled. 
Numerical Experiment (C): X forms regular solution with $\mathrm{W}$ in the liquid phase.

In the third experiment, we adopt the similar parameters as the second experiment, but we set $\Omega_{W-X}^{b c c}$ to be zero and vary $\Omega_{W-X}^{\text {liquid }}$ from $-75 \mathrm{KJ} / \mathrm{mol}$ to $25 \mathrm{KJ} / \mathrm{mol}$. Fig. 11 depicts several representative computed GB diagrams for $(\mathrm{Ni}-\mathrm{X})$ co-doped $\mathrm{W}$ at $T=1673 \mathrm{~K}$, which shows that when the addition of $\mathrm{X}$ will promote $\mathrm{GB}$ disorder in $(\mathrm{Ni}-\mathrm{X})$ co-doped $\mathrm{W}$ for a significantly negative $\Omega_{W-X}^{\text {liquid }}$. The corresponding $X_{\text {film }}$ maps are shown in Fig. 12, and it can be seen that a more negative $\Omega_{W-X}^{\text {liquid }}$ will increase the adsorption of $\mathrm{X}$ at W GBs.
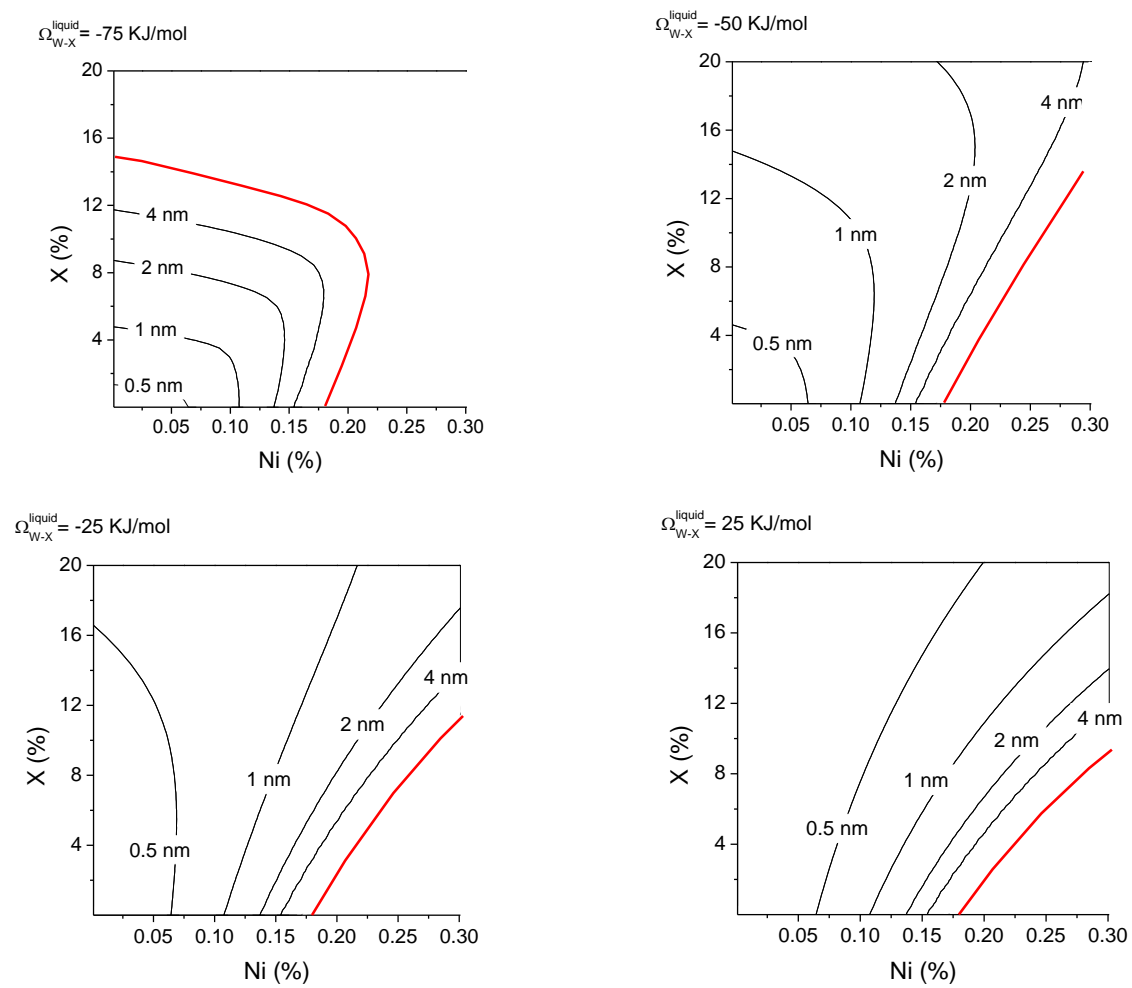

Figure 11: Computed GB $\lambda$ diagrams for the W-Ni-X system for: $\Omega_{W-X}^{b c c}=\Omega_{N i-X}^{\text {liquid }}=\Omega_{N i-X}^{b c c}=0, \mathrm{~T}=$ $1673 \mathrm{~K}$, and $T_{\mathrm{m}}{ }^{\mathrm{X}}=2000 \mathrm{~K}$. The values of $\Omega_{W-X}^{\text {liquid }}$ are labeled. 

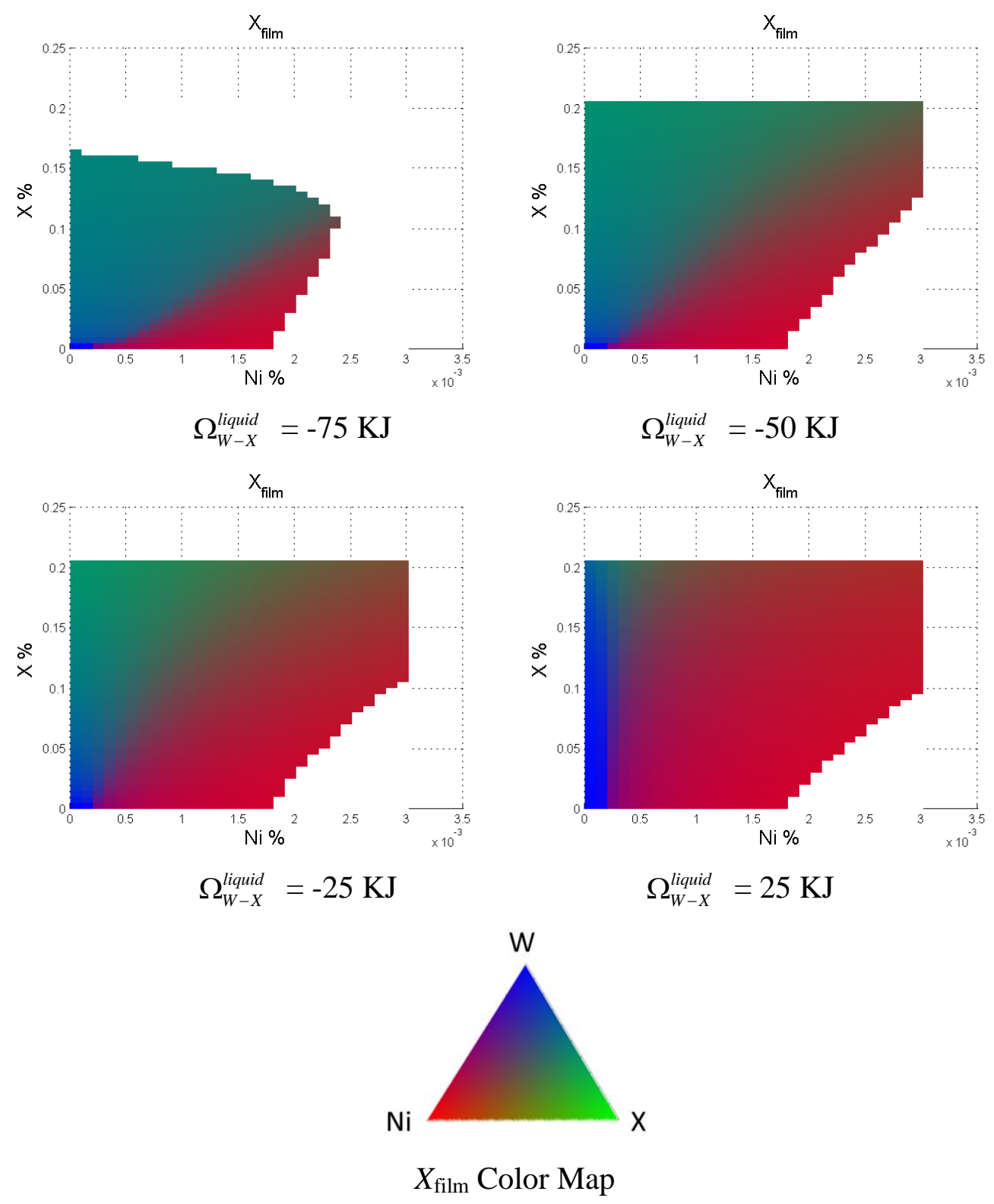

Figure 12: Computed $X_{\text {film }}$ maps for the W-Ni-X system assuming: $\Omega_{W-X}^{b c c}=\Omega_{N i-X}^{\text {liquid }},=\Omega_{N i-X}^{b c c}=0$, $\mathrm{T}=1673 \mathrm{~K}$, and $T_{\mathrm{m}}{ }^{\mathrm{X}}=2000 \mathrm{~K}$. The values of $\Omega_{W-X}^{\text {liquid }}$ are labeled. 
Numerical Experiment (D): X forms regular solutions with W in both solid and liquid phases Lastly, we modeled $\mathrm{W}-\mathrm{Ni}$-X systems assuming that $\mathrm{X}$ forms regular solutions with $\mathrm{W}$ in both solid and liquid phases (i.e., $\Omega_{W-X}^{\text {liquid }} \neq 0, \Omega_{W-X}^{b c c} \neq 0$ ), and we assume (based on Table I) that $\Omega_{W-X}^{b c c}-\Omega_{W-X}^{\text {liquid }} \approx 50 \mathrm{KJ} / \mathrm{mol}$; This because $\mathrm{X}$ is generally easier to mix with $\mathrm{W}$ in liquid phase. Fig. 13 shows their computed GB $\lambda$ diagrams for $(\mathrm{Ni}-\mathrm{X})$ co-doped $\mathrm{W}$ at $T=1673 \mathrm{~K}$, which demonstrates that the addition of $\mathrm{X}$ with greater regular-solution parameters (i.e., more difficult to mix with $\mathrm{W}$ in both solid and liquid phases) will promote GB disordering. The corresponding $X_{\text {film }}$ maps are depicted in Fig. 13.
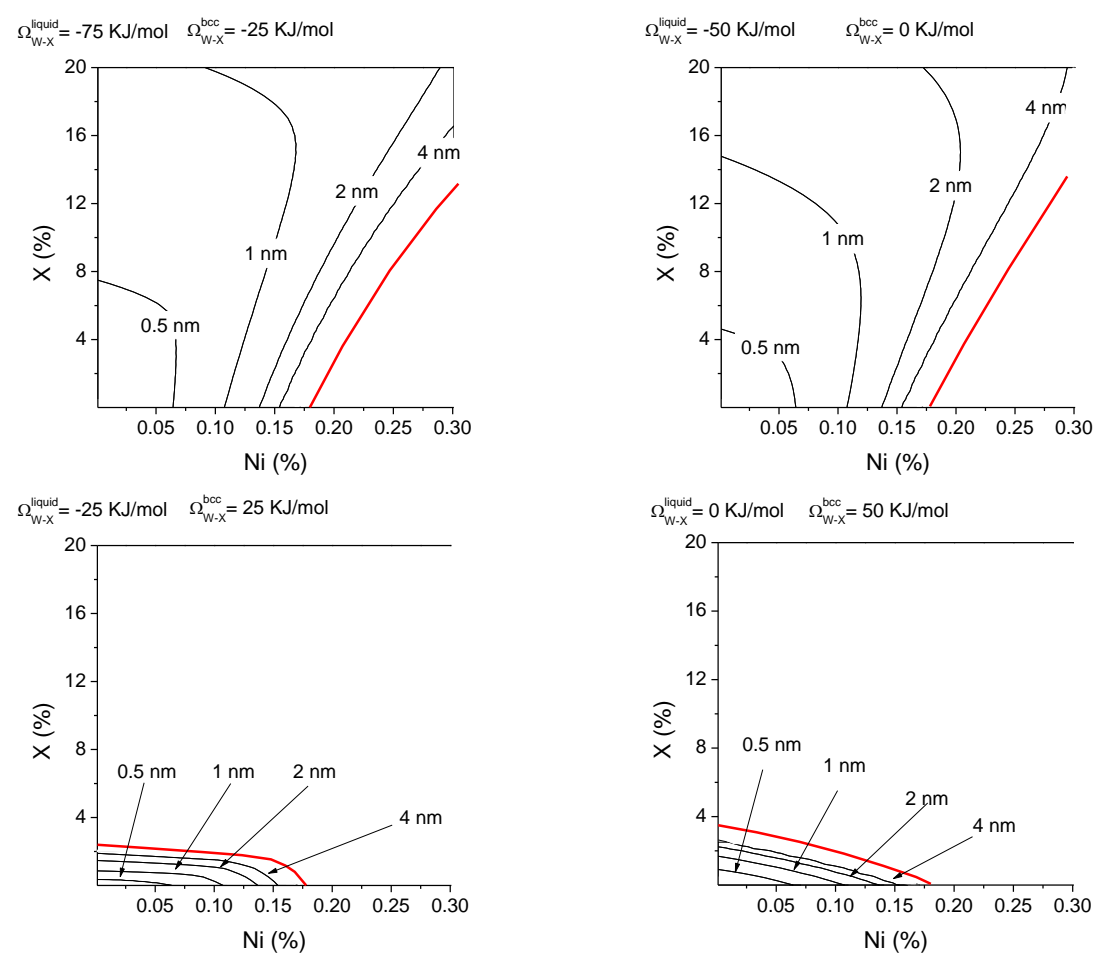

Figure 13: Computed GB $(\lambda)$ diagrams for the W-Ni-X system for: $\Omega_{N i-X}^{\text {liquid }}=\Omega_{N i-X}^{b c c}=0$, $\Omega_{W-X}^{b c c}-\Omega_{W-X}^{\text {liquid }}=50 \mathrm{KJ} / \mathrm{mol}, \mathrm{T}=1673 \mathrm{~K}$ and $T_{\mathrm{m}}{ }^{\mathrm{X}}=2000 \mathrm{~K}$. The values of $\Omega_{W-X}^{\text {liquid }}$ and $\Omega_{W-X}^{b c c}$ are labeled. 

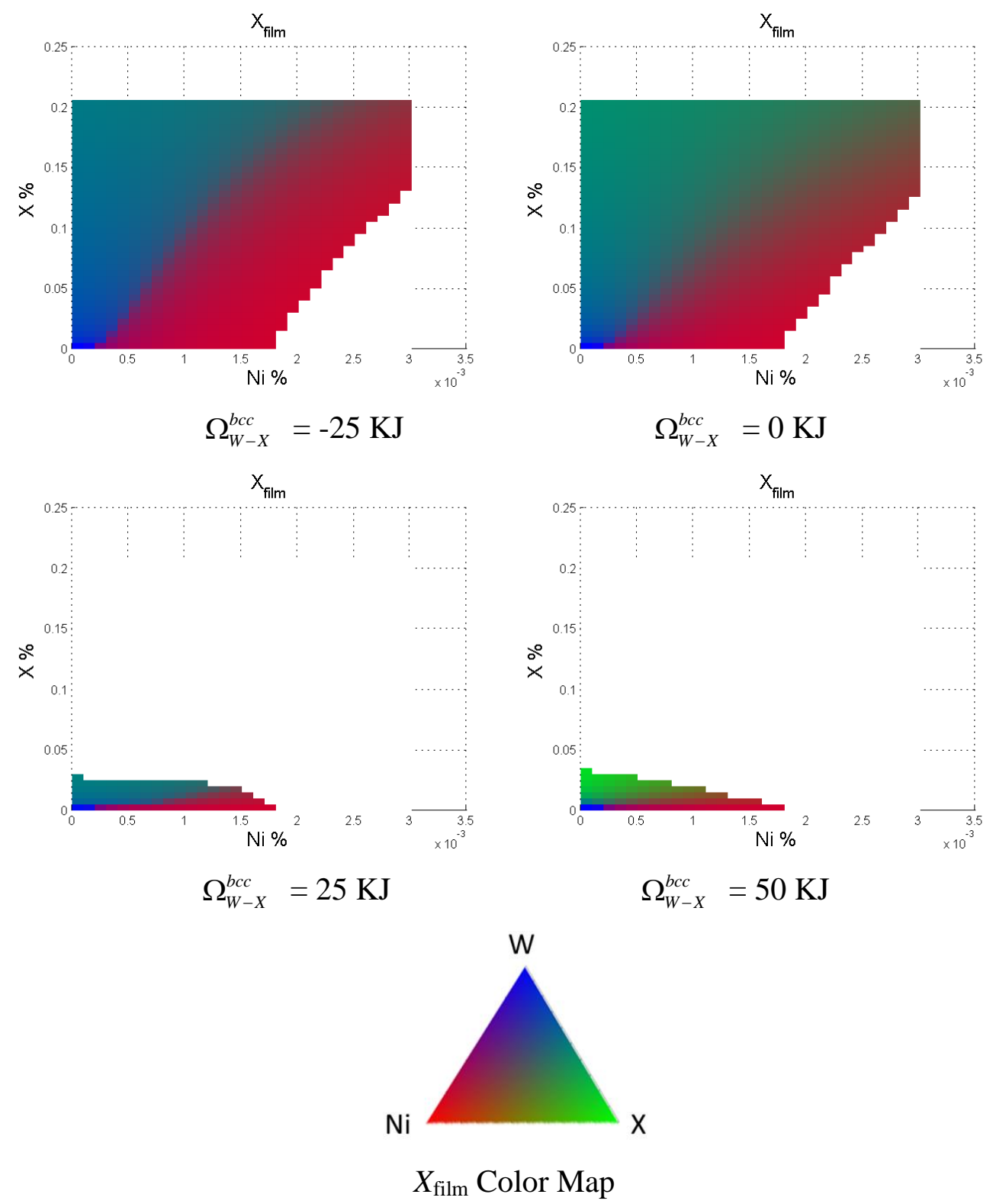

Figure 14: Computed $X_{\text {film }}$ maps for the W-Ni-X system assuming: $\Omega_{N i-X}^{\text {liquid }}=\Omega_{N i-X}^{b c c}=0$, $\Omega_{W-X}^{b c c}-\Omega_{W-X}^{\text {liquid }}=50 \mathrm{KJ} / \mathrm{mol}, \mathrm{T}=1673 \mathrm{~K}$ and $T_{\mathrm{m}}{ }^{\mathrm{X}}=2000 \mathrm{~K}$. The values of $\Omega_{W-X}^{\text {liquid }}$ and $\Omega_{W-X}^{b c c}$ are labeled.

\section{$\underline{\text { Summary }}$}

We illustrated that lowering $T_{\mathrm{m}}$ of $\mathrm{X}$, reducing $\Omega_{W-X}^{\text {liquid }}$ and increasing $\Omega_{W-X}^{b c c}$ will promote GBs in $(\mathrm{Ni}-\mathrm{X})$ co-doped $\mathrm{W}$ to disorder. We are currently conducting more systematic numerical experiments to examine the effects of other parameters as well as the combined influences of multiple parameters.

Numerical Calculation of W-Ni-X $\lambda$ Diagrams $(\mathrm{X}=\mathrm{Fe}, \mathrm{Ti}, \mathrm{Co}, \mathrm{Cr}, \mathrm{Zr}, \mathrm{Nb}$ ) 
Firstly, we modeled of W-Ni-Cr system based on existing bulk CALPHAD data ${ }^{7}$. In the ternary system, five phases, W-based BCC, Ni-based FCC, NiW, $\mathrm{NiW}_{2}$ and $\sigma$ phase (a Ni-W-Cr ternary phase), are considered. Fig. 15 showed the calculated ternary $\lambda$ diagram of this system at three different temperatures: $1000{ }^{\circ} \mathrm{C}, 1200{ }^{\circ} \mathrm{C}$ and $1400{ }^{\circ} \mathrm{C}$. $\lambda$ isothermo is drawn for small doping level at $1400{ }^{\circ} \mathrm{C}$, it can be seen that in the $\mathrm{BCC}$ phase region, adding $\mathrm{Cr}$ slightly reduces the wetability of $\mathrm{Ni}$ based liquid ar $\mathrm{W}$ grain boundraies, since the $\lambda$ contour is paralell to $\mathrm{Cr}$ Axis; however, at the slovus line (where the FCC Ni phase percipitate) adding $\mathrm{Cr}$ still promote the grain boundaryd disordering.

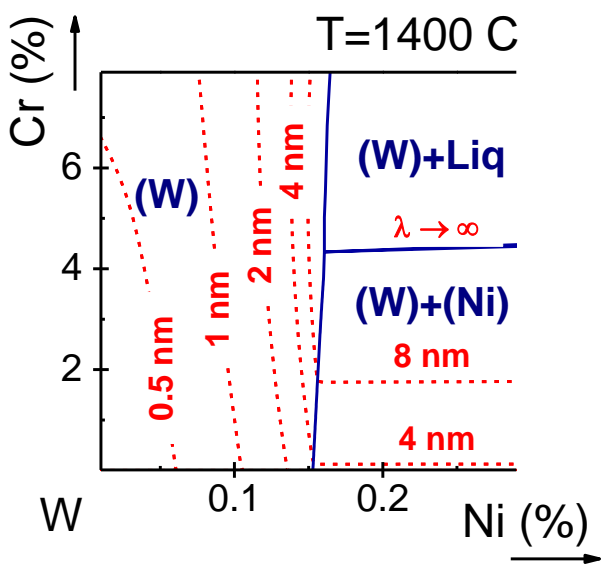

(a)

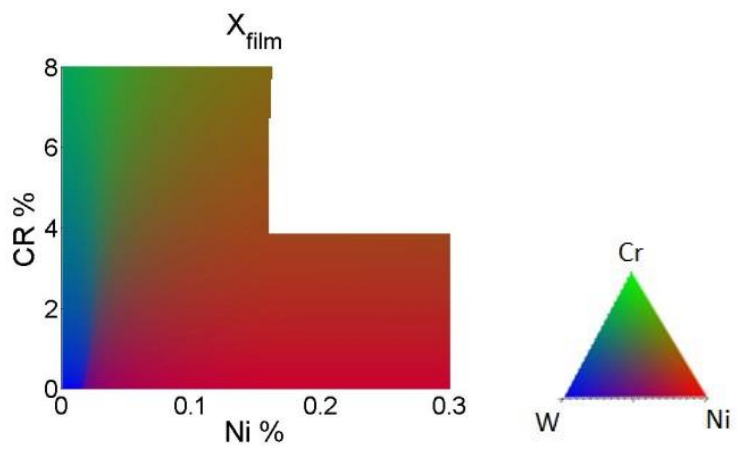

(b)

Figure 15: a) Computed $\lambda$ diagram of W-Ni-Cr system b) $X_{\text {film }}$ maps

Fig. 15 depicted the $\lambda$ and $\mathrm{X}_{\text {film }}$ diagrams for $\mathrm{W}-\mathrm{Ni}-\mathrm{Cr}$ system at $0-0.3 \% \mathrm{Ni}$ and $0-20 \% \mathrm{Cr}$. The wide composition range of these diagrams show the picture of $\lambda$ evolution at the multi-phase equilibrium region. $\lambda$ isothermo at $1000{ }^{\circ} \mathrm{C}$ (Fig. 15 a) suggests that for that the ternary dopant $\mathrm{Cr}$ has a clear dewetting effect at the $\mathrm{W}-\mathrm{Cr}$ range at $>5 \%$ while at the lower dopant level $<5 \%$ it tend to enhance GB premelting. Such effect is also observed in $\lambda$ isothermo at $1200{ }^{\circ} \mathrm{C}$. At $1400{ }^{\circ} \mathrm{C}, \mathrm{GB}$ melting is anticipated by adding $5 \% \mathrm{Cr}$ since the $\mathrm{W}$-Liquid equilibrium region appears. But if $\mathrm{Cr}$ 's composition exceeds $\sim 15 \%$, the GB could be restablized. Thus it could be generally concluded that the ternary dopant $\mathrm{Cr}$ could play both wetting or dewetting roles for GB premelting which is dependent on Cr's fraction. 

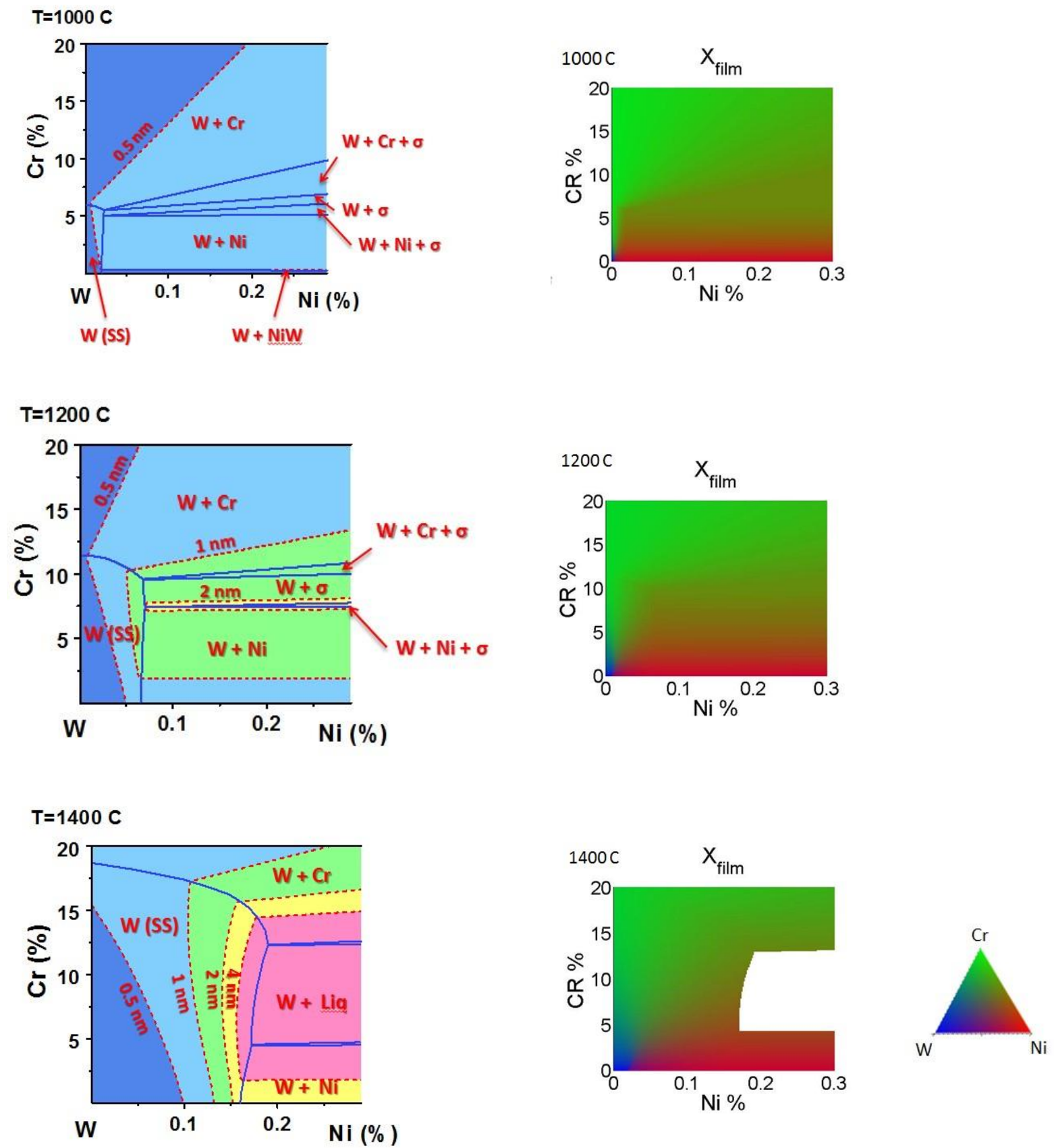

(a)

(b)

Figure 16: a) Computed $\lambda$ diagrams of W-Ni-Cr system b) $X_{\text {film }}$ maps

The thermodynamic data bases for other thernary W-Ni-X systems were built by combining binary SGTE datas. The ternary interactions are omitted. 
Table II: Regular solution parameters ( $\Omega$ 's) for chosen dopants from the SGTE binary database.

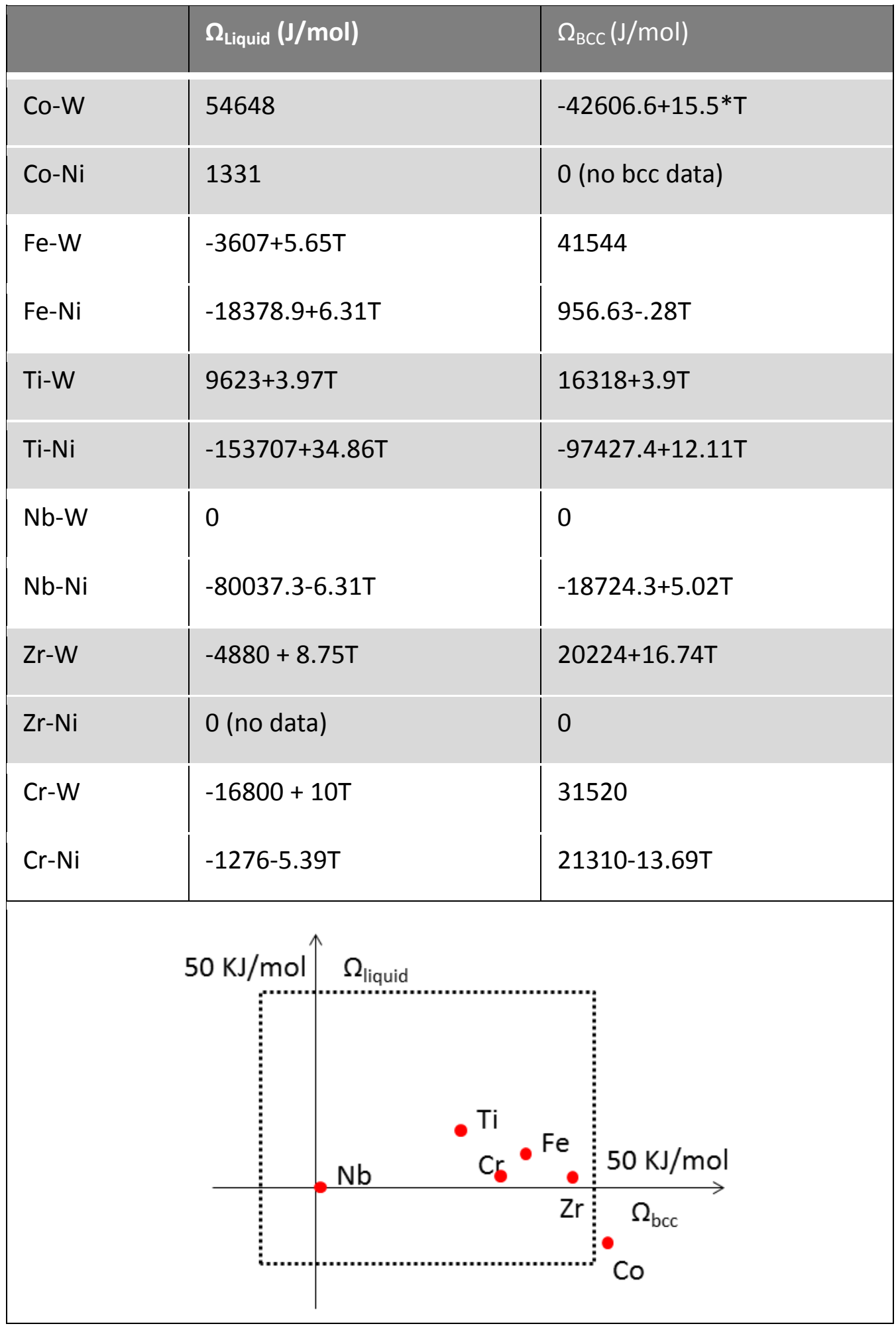


The simulation temperature is chosen as $1400{ }^{\circ} \mathrm{C}, 1600{ }^{\circ} \mathrm{C}$. We calculated the single phase $\lambda$ diagrams of these systems.
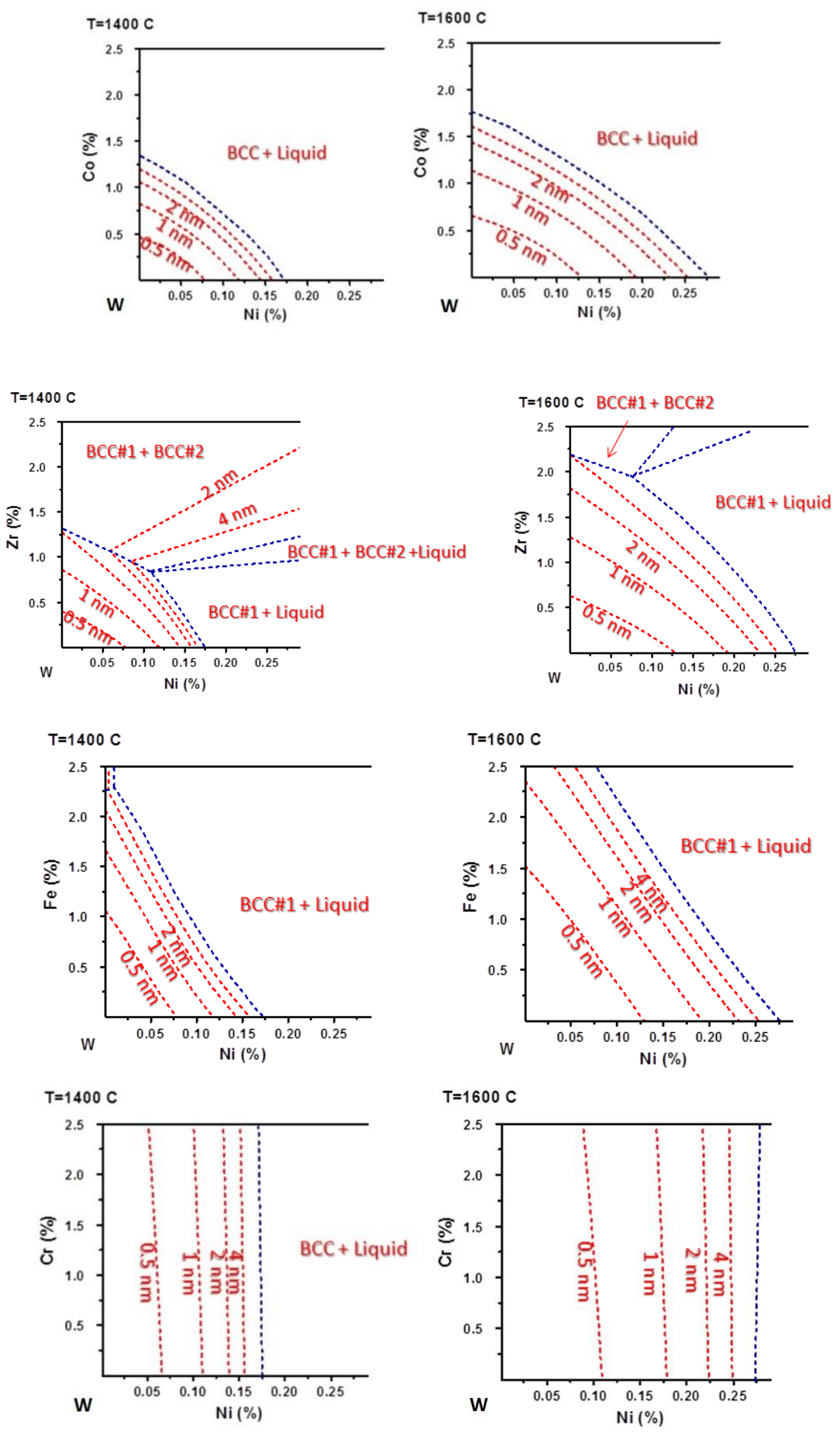

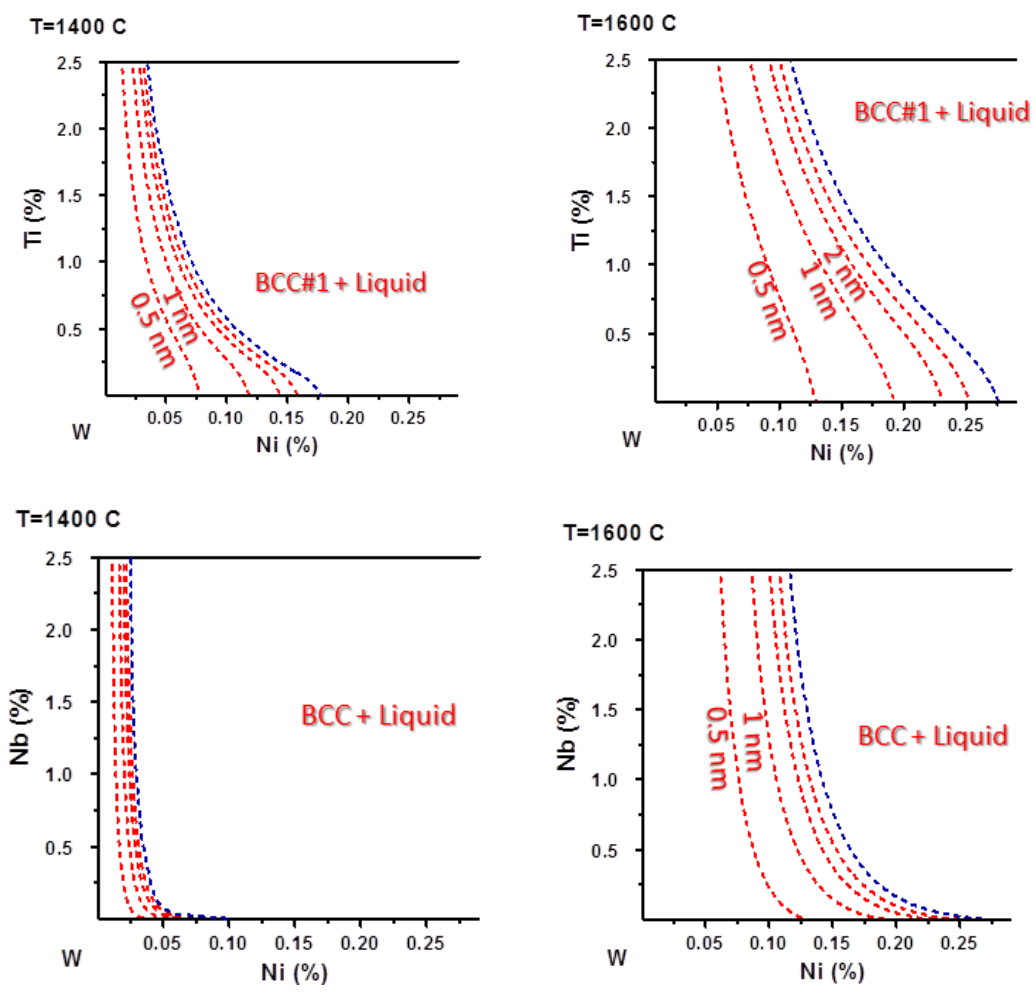

Figure 17: Computed $\lambda$ diagrams of $\mathrm{W}-\mathrm{Ni}-\mathrm{X}$ system at $1400{ }^{\circ} \mathrm{C}$ and $1600{ }^{\circ} \mathrm{C}$

The single phase $\lambda$ diagrams showed that all chosen ternary dopants tend to wet GB at bcc single phase region except for the $\mathrm{Cr}$ case which has no obvious effect. The wettability of these dopants are observed to be proportional to their bcc regular solution parameters with tungsten as Fig. 17 shows. For W-Ni-Ti and W-Ni-Nb though their X-W bcc regular solution parameters are not large, an obvious wetting tendency could be concluded at high Ni doping level which could be attributed to the a higher mixing heat of $\mathrm{Ni}-\mathrm{Ti}$ and $\mathrm{Ni}-\mathrm{Nb}$ at bcc phase than liquid phase.

For the same experiment temperatures, $\lambda$ diagrams considering multi-phase equilibrium are also obtained.
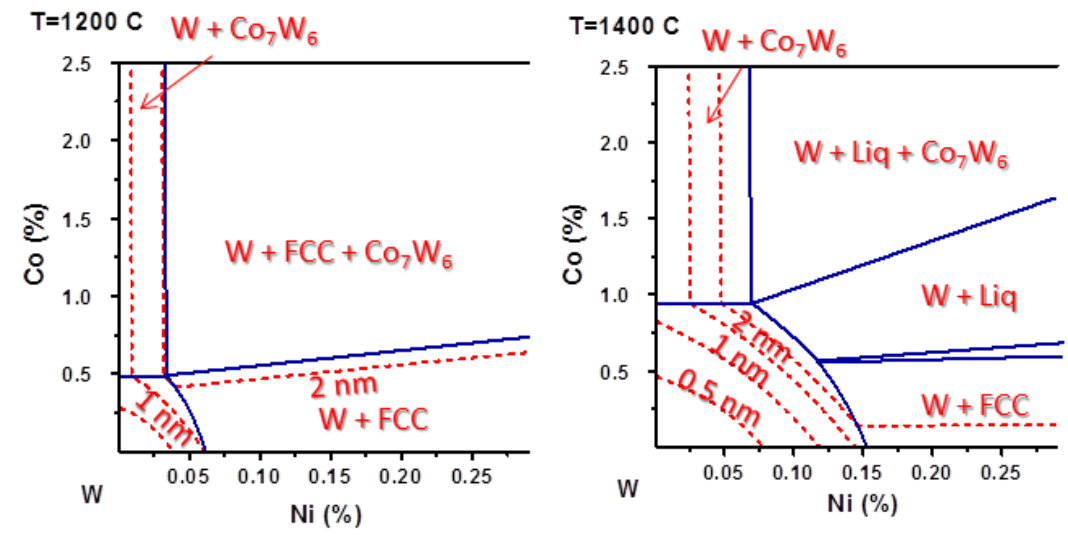

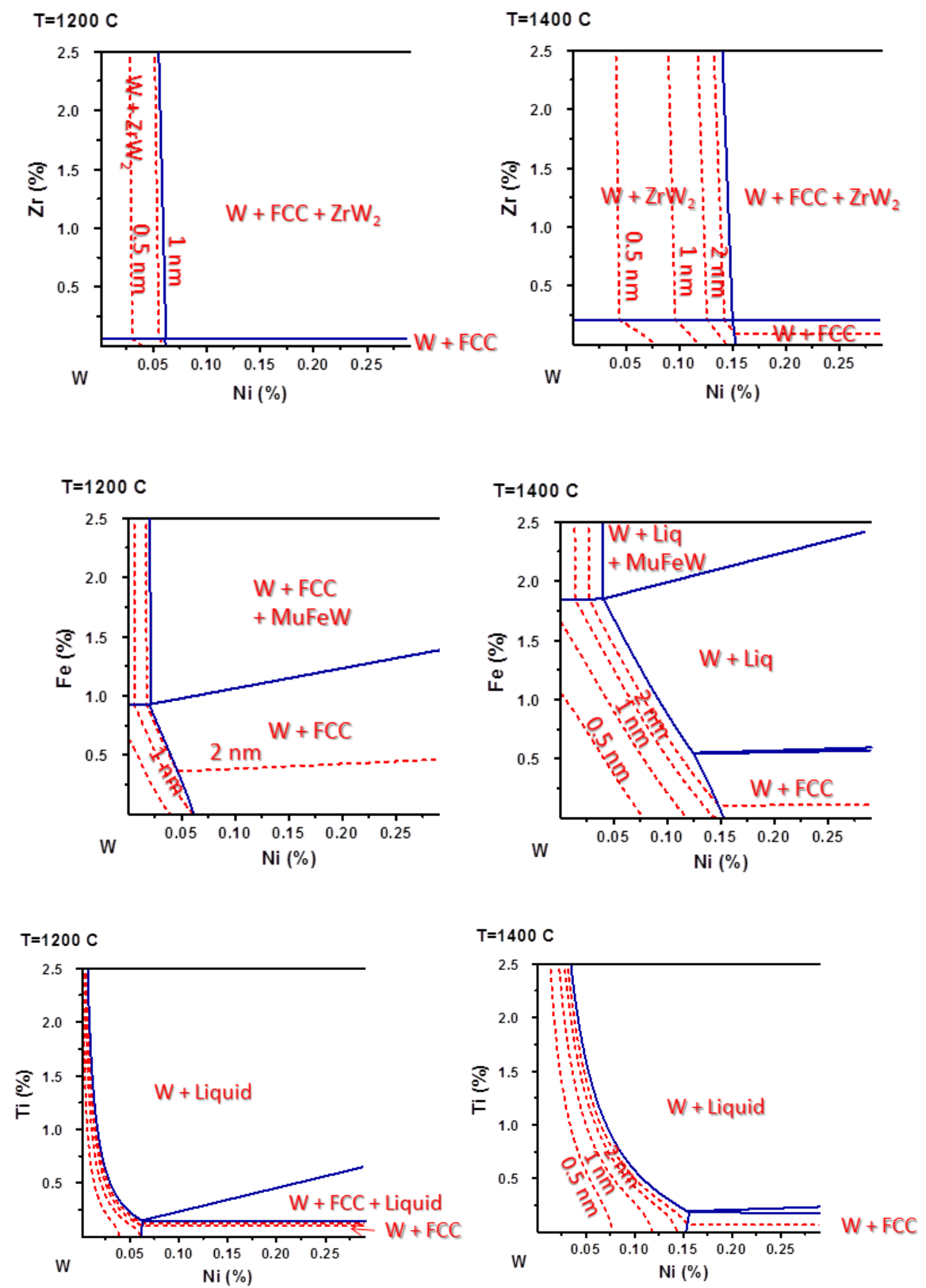

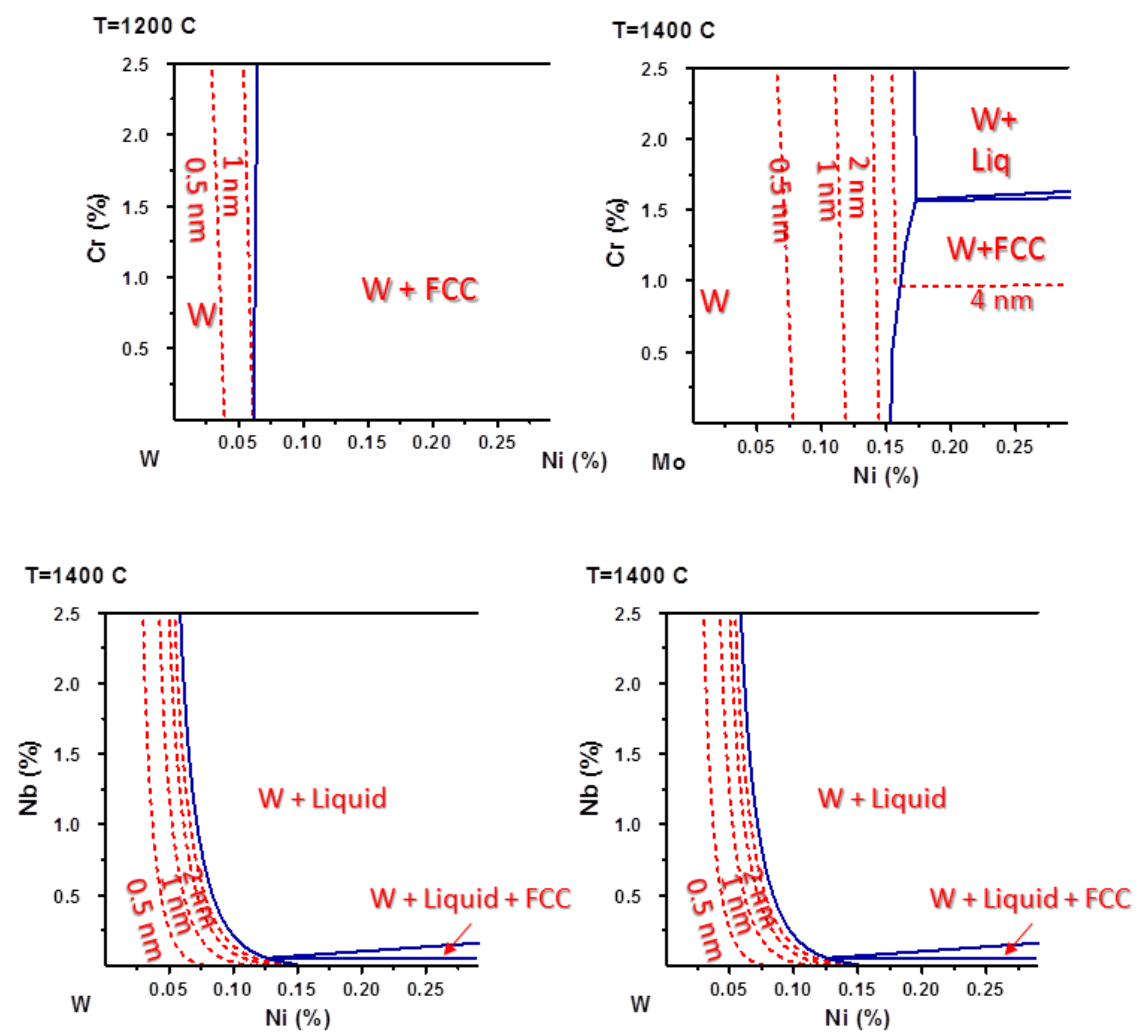

Figure 18: Computed $\lambda$ diagrams considering multi-component system of $\mathrm{W}-\mathrm{Ni}-\mathrm{X}$ system at $1400{ }^{\circ} \mathrm{C}$ and $1600{ }^{\circ} \mathrm{C}$

There are a lot of approximations applied in this set of calculation: 1. Only binary compounds are considered. 2. The ternary interactions are omitted. 3. The binary interactions of $\mathrm{Ni}-\mathrm{X}$ are assumed to be zero. 


\section{§3. Experiments Validation of Thermodynamic Theories/Modeling Results}

Experiments for validation of the simulation results have been conducted. A special quench furnace has been refurbished with new heating elements. Temperature calibration has been conducted. It is a vertical tube furnace. When the furnace was running, $\mathrm{Ar}+5 \% \mathrm{H}_{2}$ gas is flowing through the system to protect specimens from oxidation. This furnace is unique in that a mechanism of loading/unloading samples was built so that the specimens can be inserted into the hot zone of the furnace after the furnace reaches pre-set temperature. The specimens can also be taken out for gas quench after a predetermined sintering time by pulling out the crucible back to the cold-zone. The advantage is that the specimens are heated to a target temperature in a negligibly short time ( 2 minutes), so there is almost no heating ramp in beginning period. Moreover, after a certain sintering time, the specimen can be cooled down quickly (to below 800 ${ }^{\circ} \mathrm{C}$ in $\sim 1 \mathrm{~min}$ ) so that cooling ramp is also negligible. By doing this, the effects of heating and cooling ramps are minimized. In experiment, a second thermocouple can be placed in the crucible to monitor the actual temperature of the specimens. This furnace is used to make $\mathrm{W}$ based specimens for validation of our theories and multiscale modeling results.

Our specific goal is to conduct controled sintering experiments for a aseries of ternary $\mathrm{W}-0.5 \mathrm{Ni}-0.5 \mathrm{X}$ at. $\%$ (where $\mathrm{X}=\mathrm{Cr}, \mathrm{Zr}, \mathrm{Co}, \mathrm{Fe}, \mathrm{Nb}, \mathrm{Ti}$ ), along with binary $\mathrm{W}-0.5 \mathrm{Ni}$ at. $\%, \mathrm{~W}-1$

$\% \mathrm{Ni}$ alloys as reference. Since our prior studies ${ }^{2,8}$ have clearly shown that the sintering rates are correlated to the level of GB disorder, which can be represented by computed $\lambda$ values, we plan to critical model-experimental comparison between the computed $\lambda$ values and the experimentally observed sintering rates help validate our thermodynamic theories and modeling results. The experiments procedure and materials used are described below.

\section{Experiment Procedure}

1. Weighing

2. Mixing and Drying: Mix all chemical reagents in acetone; shake with alumina balls for 30 minutes and dry in an oven at $80^{\circ} \mathrm{C}$ thoroughly.

3. Calcining: Calcine the dried mixture in the Lindberg tube furnace flowing the $\mathrm{Ar}+$ $5 \% \mathrm{H}_{2}$ at $600^{\circ} \mathrm{C}$ for 1 hour, and heating rate is $5^{\circ} \mathrm{C}$.

4. Mixing and Drying: Add PMMA and SA again to calcined mixture and shake with alumina balls in acetone for 30 minutes and drying in oven at $80^{\circ} \mathrm{C}$ thoroughly.

5. Grinding and Sieving : Crush the powder and sieve the powder with the sieve of 140 mesh

6. Pressing: first compacting powder by uniaxial pressing at 225Mpa for 20 seconds and then CIP at 380Mpa for 2 minutes.

7. Presintering: Sintering in the vertical furnace flowing the $\mathrm{Ar}+5 \% \mathrm{H}_{2}$ at $900^{\circ} \mathrm{C}$ for 2 hour, and heating rate is $10^{\circ} \mathrm{C}$.

8. Sintering: First heat the furnace to the target temperature and then drop the samples from cooling zone to the heating zone; hold the temperature for scheduled time; quenching the samples in the gas.

9. Measurement: Densities are calculated by measuring the dimensions of sample after pressing, presintering and sintering. 
Table III: Raw Materials (All were purchased from Alfa Aesar except that PMMA was purchased from Lucite International and SA was purchased from Fisher Chemicals)

\begin{tabular}{|c|c|c|}
\hline Chemical Reagent & Purity & Particle Size \\
\hline $\mathrm{W}$ powder & $99.999 \%, \mathrm{Mo}<1$ & $4-6 \mu \mathrm{m}$ \\
\hline $\mathrm{NiCl} \cdot 6 \mathrm{H}_{2} \mathrm{O}$ & $98 \%$ & \\
\hline $\mathrm{Ti}$ powder & $99.5 \%$ & $150 \mu \mathrm{m}$ \\
\hline $\mathrm{CrCl}_{3}$ & $99.9 \%$ & \\
\hline $\mathrm{Cl}_{2} \mathrm{Fe}$ & $99.5 \%$ & \\
\hline $\mathrm{ClCo}_{2} \cdot 6 \mathrm{H}_{2} \mathrm{O}$ & $99.9 \%$ & \\
\hline $\mathrm{ZrCl}_{4}$ & $99.5 \%$ & \\
\hline $\mathrm{NbCl}_{5}$ & $99.95 \%$ & \\
\hline Acrylic Resins (PMMA) & & \\
\hline Stearic Acid (SA) & & \\
\hline
\end{tabular}

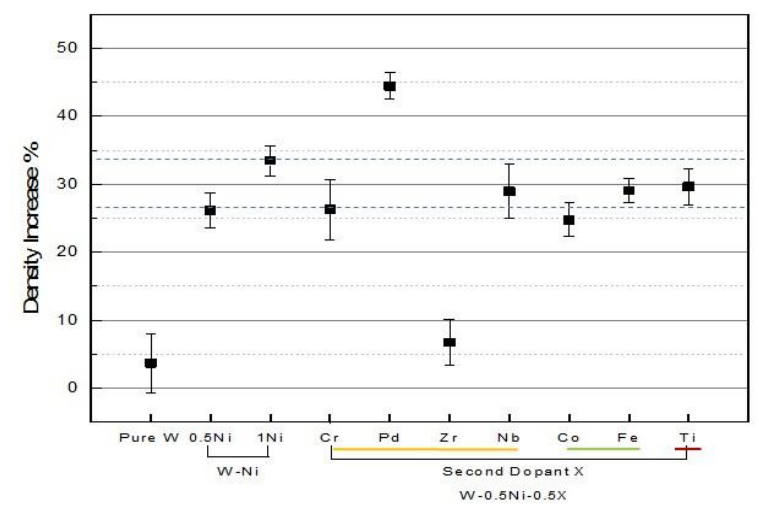

(a)

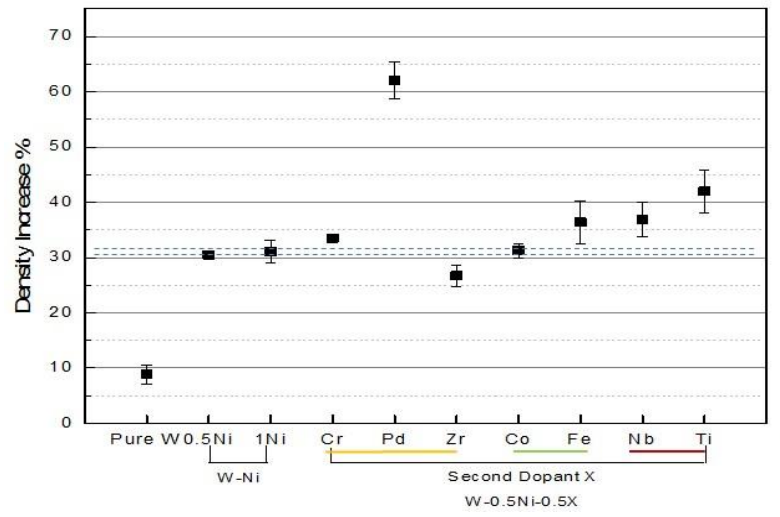

(b)

Figure 19: Experimental Data: (a) average density increase of $\mathrm{W}-0.5 \mathrm{Ni}-0.5 \mathrm{X}$ at. $\%(\mathrm{X}=\mathrm{Cr}$, $\mathrm{Zr}, \mathrm{Co}, \mathrm{Fe}, \mathrm{Nb}, \mathrm{Ti}$ ) specimens, sintered at $1473 \mathrm{~K}$ for 5 hours and (b) average density increase of $\mathrm{W}-0.5 \mathrm{Ni}-0.5 \mathrm{X}$ at. $\%(\mathrm{X}=\mathrm{Cr}, \mathrm{Zr}, \mathrm{Co}, \mathrm{Fe}, \mathrm{Nb}, \mathrm{Ti})$ specimens sintered at $1573 \mathrm{~K}$ for 2 hours.

To eliminate the influence of different pre-sintered densities and show the effectiveness of different dopants more precisely, we use average density increase percentage to present our results (Fig. 19). In Fig. 20, we re-plot that data shown in Fig. 19(b), along with our computed $\lambda$ values extracted from the GB diagrams presented in the prior section $(\$ 2.2)$ for a critical model-experimental comparison (Figure 19). 


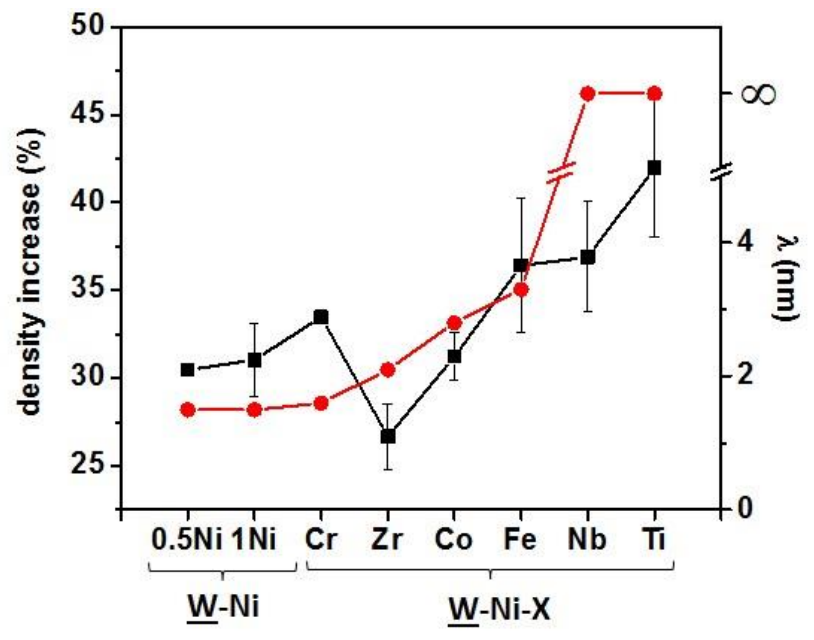

Figure 20: A Critical Model-Experimental Comparison: Computed $\lambda$ values (extracted from the GB diagrams presented in \$2.2) vs. the experimentally-observed sintering rates (density increases after sintering at $1573 \mathrm{~K}$ for 2 hours) of ternary $\mathrm{W}-0.5 \mathrm{Ni}-0.5 \mathrm{X}$ at. $\%(\mathrm{X}=\mathrm{Cr}, \mathrm{Zr}, \mathrm{Co}$,

$\mathrm{Fe}, \mathrm{Nb}, \mathrm{Ti}$ ), along with binary $\mathrm{W}-0.5 \mathrm{Ni}$ at. $\%, \mathrm{~W}-1 \% \mathrm{Ni}$ alloys as reference. The experimentally-observed sintering rate is an indication of the level of GB disorder. Thus, the correlation between the computed $\lambda$ values and the experimentally-observed sintering rates help validate our thermodynamic theories/modeling.

In summary, Fig. 20 clearly showed that our model predictions (calculated $\lambda$ values) are consistent with experimental observations (sintering rates as indications of the level of GB disorder). Some specific discussion is given below. At $1573 \mathrm{~K}$, The density increases of W-0.5Ni (30\%) and $\mathrm{W}-1 \mathrm{Ni} \mathrm{(31 \% )} \mathrm{are} \mathrm{rather} \mathrm{close.} \mathrm{The} \mathrm{density} \mathrm{increases} \mathrm{of} \mathrm{Co}, \mathrm{Cr}, \mathrm{Fe}, \mathrm{Nb}$ and $\mathrm{Ti}$ are higher than $\mathrm{W}-1 \mathrm{Ni}$. Ti has the best effectiveness, of which density increase is $42 \%$. The measured density increases are generally in consistent with the $\lambda$ calculation. But the density increase of $\mathrm{W}-0.5 \mathrm{Ni}-0.5 \mathrm{Zr}(27 \%)$ is lower than $\mathrm{W}-0.5 \mathrm{Ni}(31 \%)$. Such negative effect of $\mathrm{Zr}$ on the system may be explained by the reduction of sintering driving force (sintering powder's surface energy) with Zr's doping. Our calculation using Wynblatt surface segregation model ${ }^{9}$ shows that $\mathrm{Zr}$ has the strongest effect on decreasing the surface energy of $\mathrm{W}$ comparing with other dopants ( $30 \%$ at $0.5 \%$ doping level). Overall, the model-experimental comparison is rather satisfactory. 


\section{Part B (\$4-6) Multiscale Modeling (Performed by the Purdue team)}

Another important effort of this study is to use a combination of thermodynamic modeling and experiments to supply information for the QM and multiscale simulation of Purdue University.

Firstly, we considered $\mathrm{W}-\mathrm{Ni}$ binary systems of specimens that were equilibrated (quenched from) $1400{ }^{\circ} \mathrm{C}$ of different nominal bulk composition. The computed $\lambda$ diagram ( $\lambda$ value vs. bulk composition for an isothermal section at $1400^{\circ} \mathrm{C}$ ) provides an estimated interfacial width and film composition vs. bulk composition. These thermodynamic predictions are then validated with experimental data of interfacial width and composition from HRTEM and Auger (with depth profiling), and adjustments are made. Then such validated data on interfacial width and film (grain boundary) composition are supplied to Purdue University and used in their quantum-atomistic simulations, then the simulation results will further provide data that are useful for continuum level fracture simulation.

Here, we recognize that two precautions, as follows:

- The computed $\lambda$ value overestimates film thickness (by its definition), and the true interfacial width is somewhat uncertain because of the difficulty in quantifying the interfacial (disjoining) potential.

- The measured Ni/W ratio (by Auger electron spectroscopy) underestimates the actual Ni composition because 1) Auger electrons come from a few atomic layers and 2) the specimen intergranular fracture is not ideal.

Thus, we combine thermodynamic modeling and experiments to give our best estimates interfacial width and grain boundary chemistry, which will be used in quantum-atomistic (CP MD) simulations.

\section{§4. Investigation of Interfacial Property of Ni-doped W Using Quantum Simulation}

Nickel-doped tungsten has been widely studied since it was considered as a refractory metal for activated sintering. Due to tungsten's high melting point and it is not feasible to cast tungsten ingots. However, tungsten powder is usually mixed with nickel powder for sintering. A heavy alloy can be produced by the sintering process which involves the nickel diffusion into the tungsten. During the sintering process, nickel is usually found to segregate at tungsten grain boundary (GB). I.J.Toth and N.A. Lockington reported that the most of nickel was found at the surface of the tungsten powder and along the $\mathrm{GB}^{10}$. Also, T.G. Nieh presented an evidence of GB segregation of nickel in tungsten using Auger Electron Spectroscopy (AES). They also demonstrated that the nickel segregation results in a severe embrittlement of tungsten filaments. The thickness of the layer of the nickel-rich phase found to be an important factor to overcome the brittleness of the nickel activated sintered tungsten. While the amount of the nickel addition is found to be a big factor that affects material properties, N.M. Hwang and et al demonstrated comparison between pure tungsten and nickel-doped tungsten for GB by experimental study ${ }^{12}$. Y. Mishin and et al performed simulations to confirm that GB mobility depends on the GB crystallography, the temperature, and the impurity content in the material ${ }^{13}$. N.M. Hwang also suggested that the solid-state activated sintering of tungsten is due to a GB roughening transition induced by nickel segregation, where the atomic level GB structures have not been characterized. 
For conducting the numerical simulation based on continuum mechanics in meso-scale the interface between the grains need to be considered in polycrystalline materials. However, interface has very small thickness and is difficult to measure the properties in experiment or large scale simulation.

In this study, the interface of nickel-doped tungsten is presented in the atomic scale model for quantum calculation. Sintered tungsten is visualized in atomic scale with a representation of two differently oriented tungsten phases and nickel-rich GB phase. The amount of nickel in the GB phase is varied for finding the role of nickel contents to the properties in the atomic scale. A number of model sets is undergone the simulation using Car-Parrinello Molecular Dynamics (CPMD). Computational chemistry software, NWChem, is used for calculation. The NWChem plane-wave (NWPW) module is a module that uses pseudopotential plane-wave basis sets to perform Density Functional Theory calculations. This module has capabilities keeping accuracy as good as the traditional Gaussian function-based approaches and fast enough to manage hundreds of atoms. Another advantage is its ability to simulate dynamics on a ground state potential surface directly at run-time using the Car-Parrinello algorithm. This method's efficiency and accuracy make it a good candidate for first principles method of simulation in the study of complex molecular system.

CPMD is a method to find the motion of atomic nuclei in time for performing molecular dynamics (MD), while calculating ab-initio and the quantum mechanical motion of electrons. In CPMD the core electrons are usually described by pseudopotential. The wavefunction of the valence electrons are approximated by a plane wave basis set. Car and Parrienello introduced the CPMD approach which has concept of the quantum-mechanical adiabatic time-scale separation of fast electronic and slow nuclear motion by transforming that into classical mechanical adiabatic energy-scale separation in the framework of dynamical systems theory. Car and Parrinello suggested the following class of Lagrangians.

$$
L_{C P}=\sum_{I} \frac{1}{2} M_{I} \dot{R}_{I}^{2}+\sum_{i} \frac{1}{2} \mu_{i}\left\langle\dot{\psi}_{i} \mid \dot{\psi}_{i}\right\rangle-\left\langle\Psi_{0}\left|H_{e}\right| \Psi_{0}\right\rangle+\text { constraints }
$$

The first and second terms on the right side are expression of the kinetic energy and the third term is the potential energy. The last term explains the orthonormality.

The corresponding Newtonian equations of motion are obtained from the associated Euler-Lagrange equations.

$$
\begin{aligned}
& \frac{d}{d t} \frac{\partial L}{\partial \dot{R}_{I}}=\frac{\partial L}{\partial R_{I}} \\
& \frac{d}{d t} \frac{\delta L}{\delta \dot{\psi}_{i}^{*}}=\frac{\delta L}{\delta \psi_{i}^{*}}
\end{aligned}
$$

Following this ideas, generic Car-Parrinello equations of motion are found to be of the form 


$$
\begin{aligned}
& M_{I} \ddot{R}_{I}(t)=-\frac{\partial}{\partial R_{I}}\left\langle\Psi_{0}\left|H_{e}\right| \Psi_{0}\right\rangle+\frac{\partial}{\partial R_{I}} \text { \{constraints } \\
& \mu_{i} \ddot{\psi}_{i}(t)=-\frac{\delta}{\delta \psi_{i}^{*}}\left\langle\Psi_{0}\left|H_{e}\right| \Psi_{0}\right\rangle+\frac{\delta}{\delta \psi_{i}^{*}}\{\text { constraints }\}
\end{aligned}
$$

where $\mu_{i}(=\mu)$ are the "fictitious masses" or inertia parameters assigned to the orbital degrees of freedom.

Applications for this first principles method include the calculation of free energies, explicit simulation of solvated molecules, and simulations of complex vibrational modes that cannot be described within the harmonic approximation. Another advantage is its ability to simulate dynamics on a ground state potential surface directly at run-time using the Car-Parrinello algorithm. This method's efficiency and accuracy make it a desirable first principles method of simulation in this study.

Before conducting the simulations, there are several parameters need to be verified by convergence study. The number of k-points for integration over Brillouin zone is decided with convergence study using temperature stabilization as a function of time. As it is shown in Figure 25 a case of $40 \times 40 \times 40 \mathrm{k}$-point for integration appears to be most efficient and effective to obtain the result

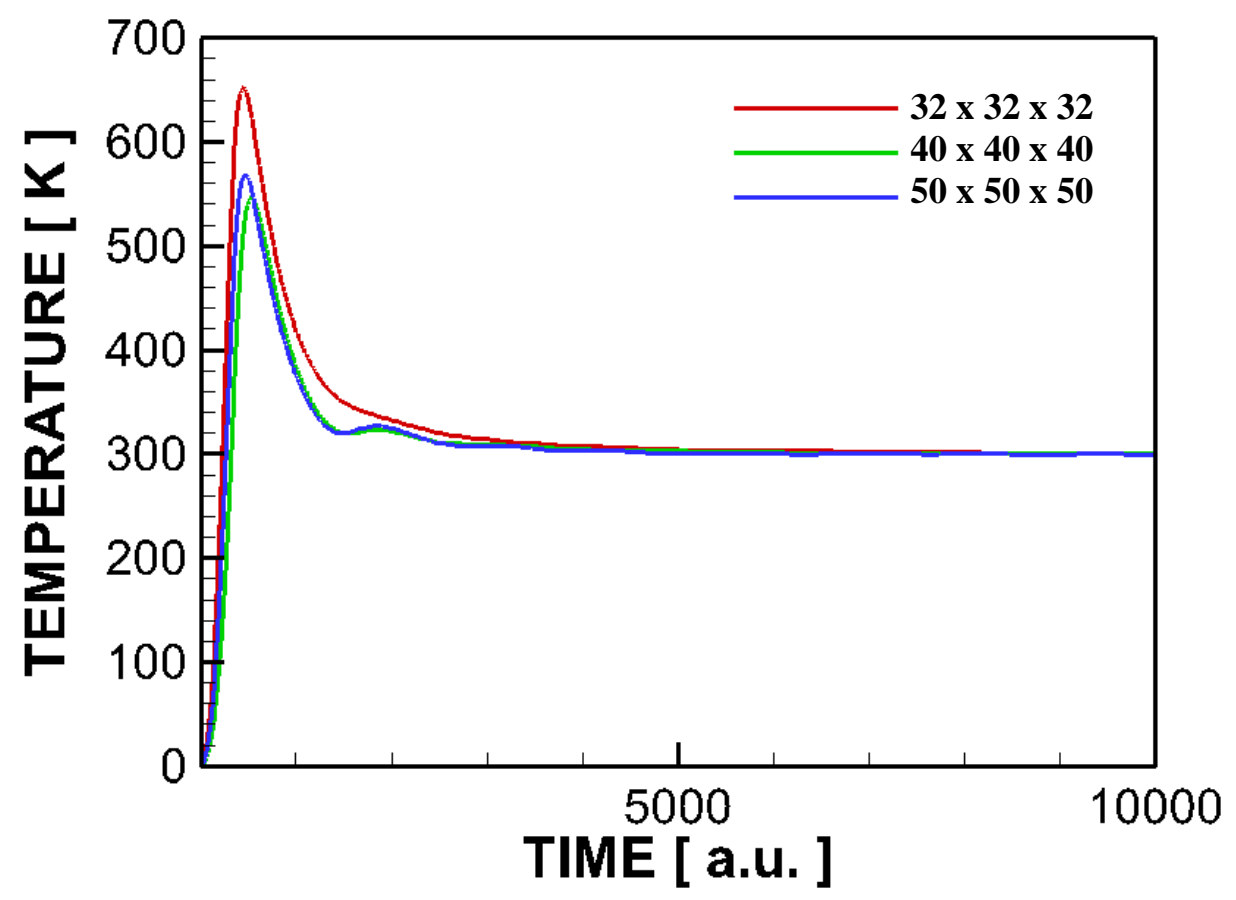

Figure 21: Convergence study on the number of k-point for integration over Brillouin zone

For the verification of the convergence study on the number of k-point for integration, comparing the atomic bond length with theoretically ideal bond length can be a reasonable method. The ideal bond length of W-W is found to be 2.81 Angstrom and W-Ni is found to be 2.86 
Angstrom. In this figure, the atomic bond length from the simulations are plotted for describe the distribution on bond length of $\mathrm{W}-\mathrm{W}$ and $\mathrm{W}-\mathrm{Ni}$ pairs.
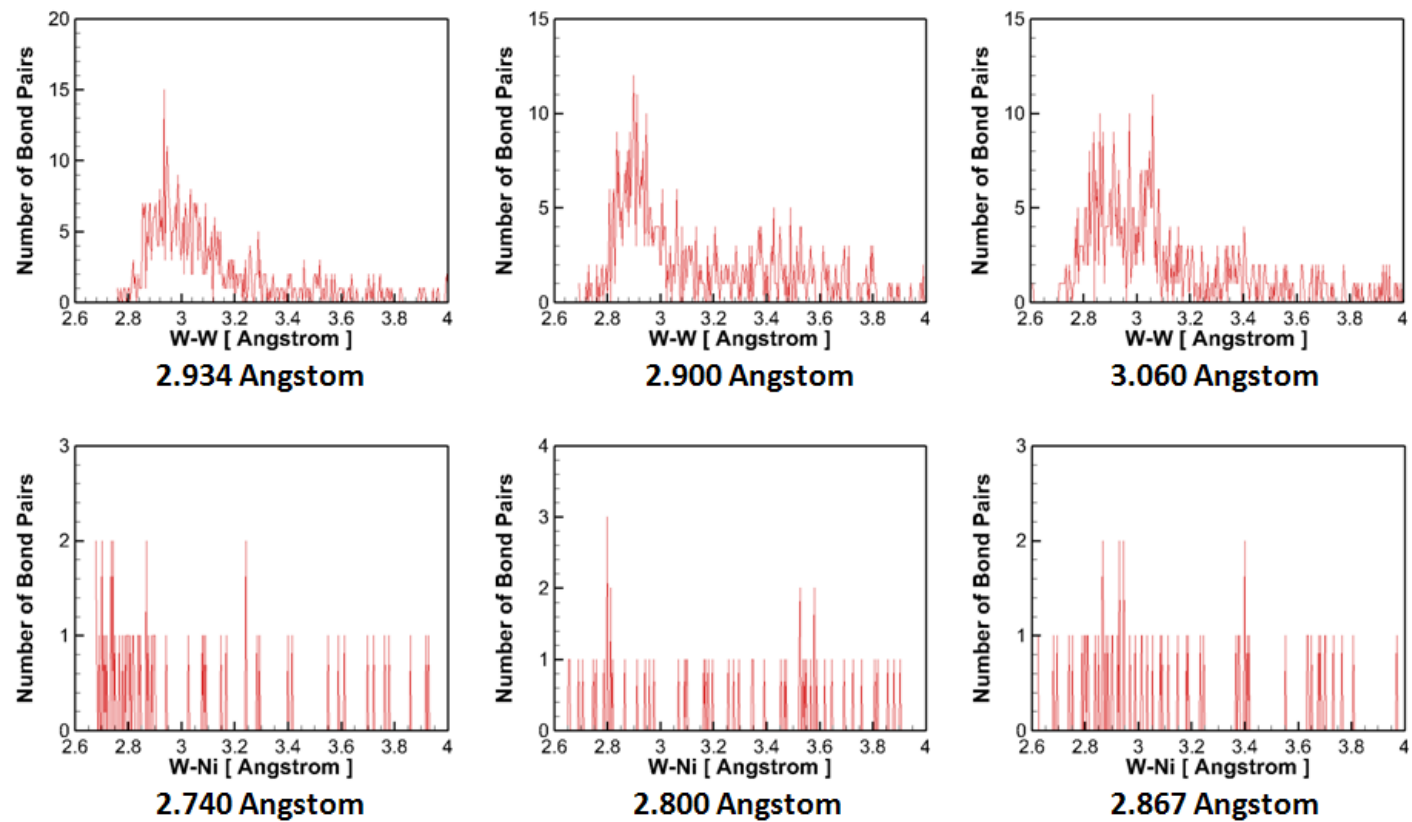

(a)

(b)

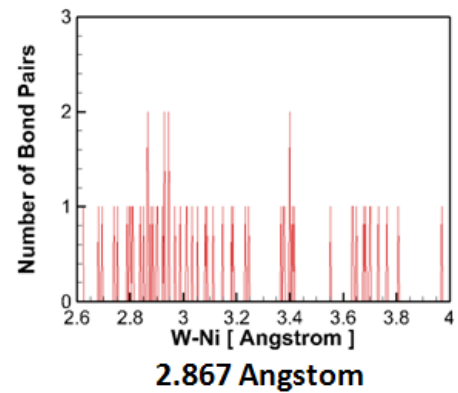

(c)

Figure 22: Distribution of bond lengths for $\mathrm{W}-\mathrm{W}$ and $\mathrm{W}-\mathrm{Ni}$ over different number of k-point

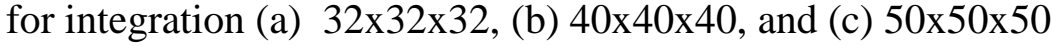

For the modeling of atomic scale nickel-doped tungsten, an image of high resolution transmission electron microscopy (HRTEM) was taken as a role model. The HRTEM image includes a representative $\mathrm{GB}$ in a $\mathrm{W}+\mathrm{Ni}$ specimen sintered at $1400^{\circ} \mathrm{C}$, which is $95^{\circ} \mathrm{C}$ below the bulk eutectic temperature. In this figure, (a) shows the HRTEM image which has approximately $0.6 \mathrm{~nm}$ thick intergranular film that contains small amount of nickel. Two different orientations of tungsten phases are clearly identified. Accordingly, the crystal structure model are developed following this pattern, which has orientations of (100) and (210) for two phases. Between these two phases, nickel added tungsten GB is modeled which has thickness of the two times of tungsten's lattice parameter (=3.1585 Angstroms). Figure 23(b) shows schematic illustration of the developed model for the simulation. 


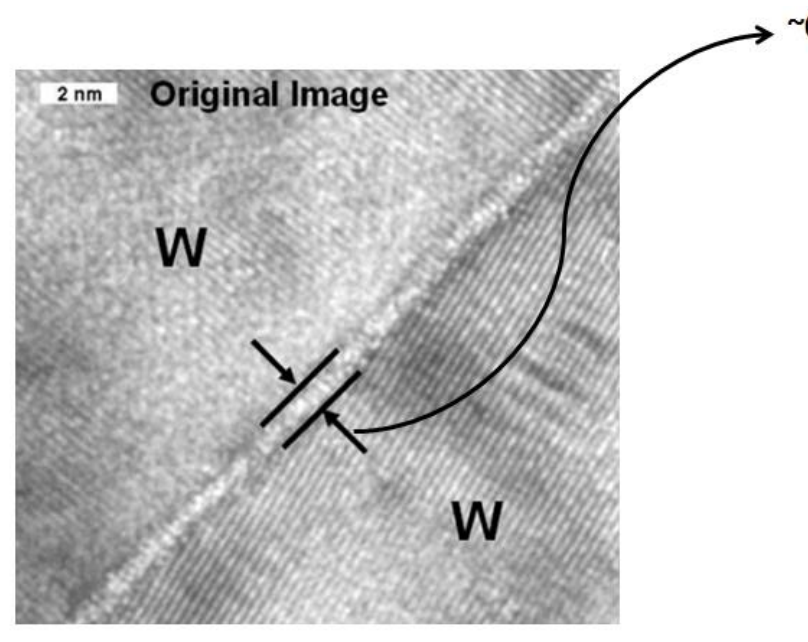

(a)

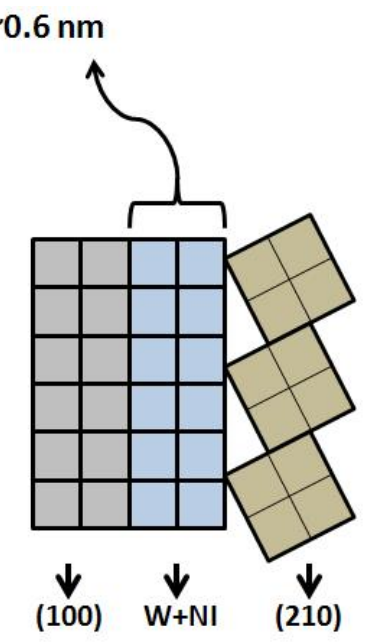

(b)

Figure 23: (a) High resolution transmission electron microscopy image of nickel-doped tungsten and (b) schematic illustration of crystal structure which represents two differently oriented tungsten phases with nickel added grain boundary

Figure 24(a), (b), (d) show the atomic structure representations of Ni-doped W in the HRTEM image which has $0 \mathrm{~nm}$ thickness for pure $\mathrm{W}, 0.3 \mathrm{~nm}$ thickness for unsaturated, and $0.6 \mathrm{~nm}$ thickness for saturated Ni-doped W. Two different orientations of W phases are clearly identified. Accordingly, the crystal structure model are developed following this pattern, which has orientations of (100) and (210) for two phases. 


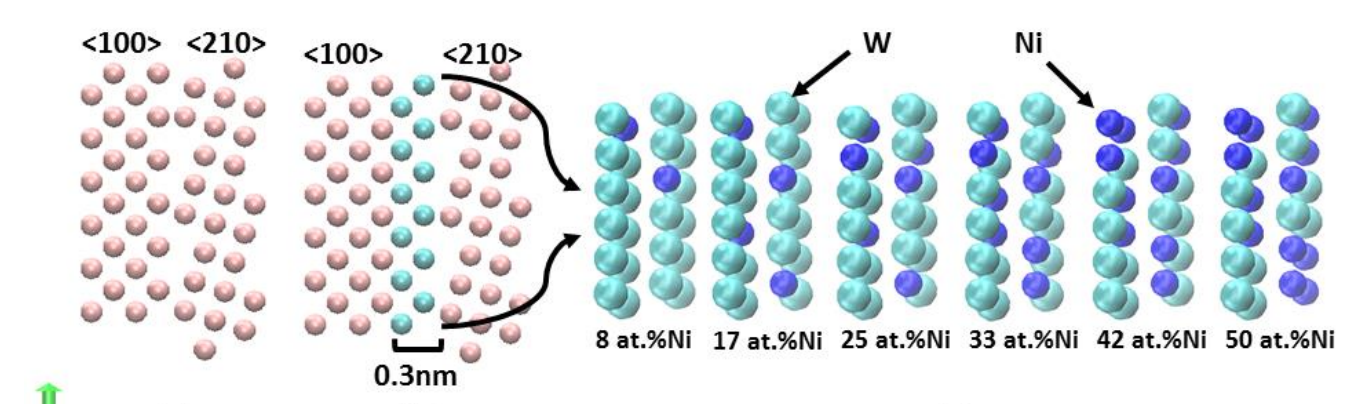

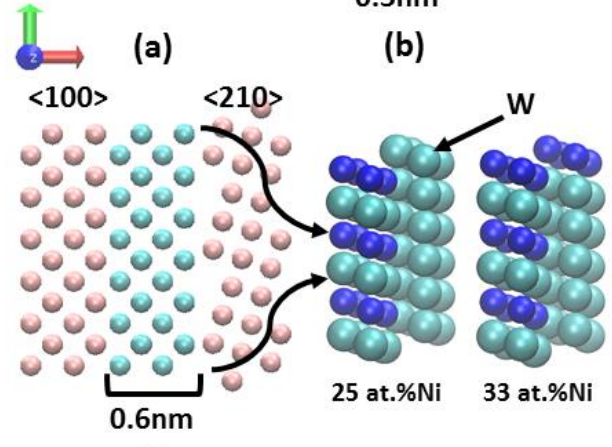

(d) (c)
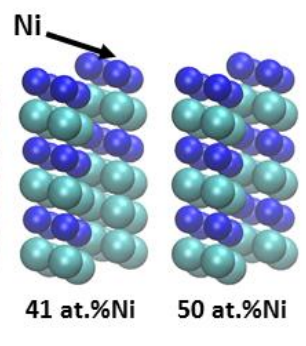

(e)

Figure 24: Illustration of atomic supercells analyzed in the present work with (a)two differently oriented $\mathrm{W}$ phases, (b) two differently oriented $\mathrm{W}$ phases with Ni added GB thickness of $0.3 \mathrm{~nm}$, (c) illustration of different atomic fractions of $\mathrm{Ni}$ in $\mathrm{W} \mathrm{GB}$, (d) illustration of atomic supercells with two differently oriented W phases with Ni added GB thickness of $0.6 \mathrm{~nm}$, (e)

illustration of different atomic fraction of $\mathrm{Ni}$ in $\mathrm{W}$ GB

The atomic model contains 96 144 atoms based on their W type with each phase having 24 (2 $\mathrm{x} 2$ x 6) unit cells. After the modeling of atomic structure it is then stretched in $\mathrm{x}$-direction with different values of strain. By applying different values of strains to the model the stress can be obtained respect to the each strain values. Strain values with interval of $2 \%$ are applied to the model. The atomic structure that forms the GB follows the structure of W with (100) orientation and a number of $\mathrm{W}$ atoms is replaced with $\mathrm{Ni}$ atoms in random manner. The GB structure is taken from the whole model and the Ni volume fraction is applied from $8 \sim 50$ at.\% Ni for the unsaturated $\mathrm{W}$ (GB thickness $=0.3 \mathrm{~nm}$ ) and $25 \sim 75$ at. $\% \mathrm{Ni}$ for the saturated $\mathrm{W}$ (GB thickness $=0.6 \mathrm{~nm}$ ) as shown in the figure $24(\mathrm{c}),(\mathrm{e})$.

Developed atomic structures have been undergone the computational study with series of strain values interval of $2 \%$ are applied. For each strain values, corresponding stress are coupled to obtain the stress-strain relation. From the obtained stress-strain curve, the yield stress and the maximum stress are predicted. The yield stress is predicted based on the $2 \%$ offset method and the corresponding strain value is observed to be at $8 \%$. For the verification purpose, pure $\mathrm{W}$ structure has undergone the simulation to compare the ultimate tensile strength with the previous data. The ultimate tensile strength appears to be $4.0 \mathrm{GPa}$ at the strain value of $16 \%$. While the tensile strength of pure $\mathrm{W}$ is known to be in the range of $1.5 \mathrm{GPa} \sim 4.2 \mathrm{GPa}$ the result from this computation is reasonable to hold. 


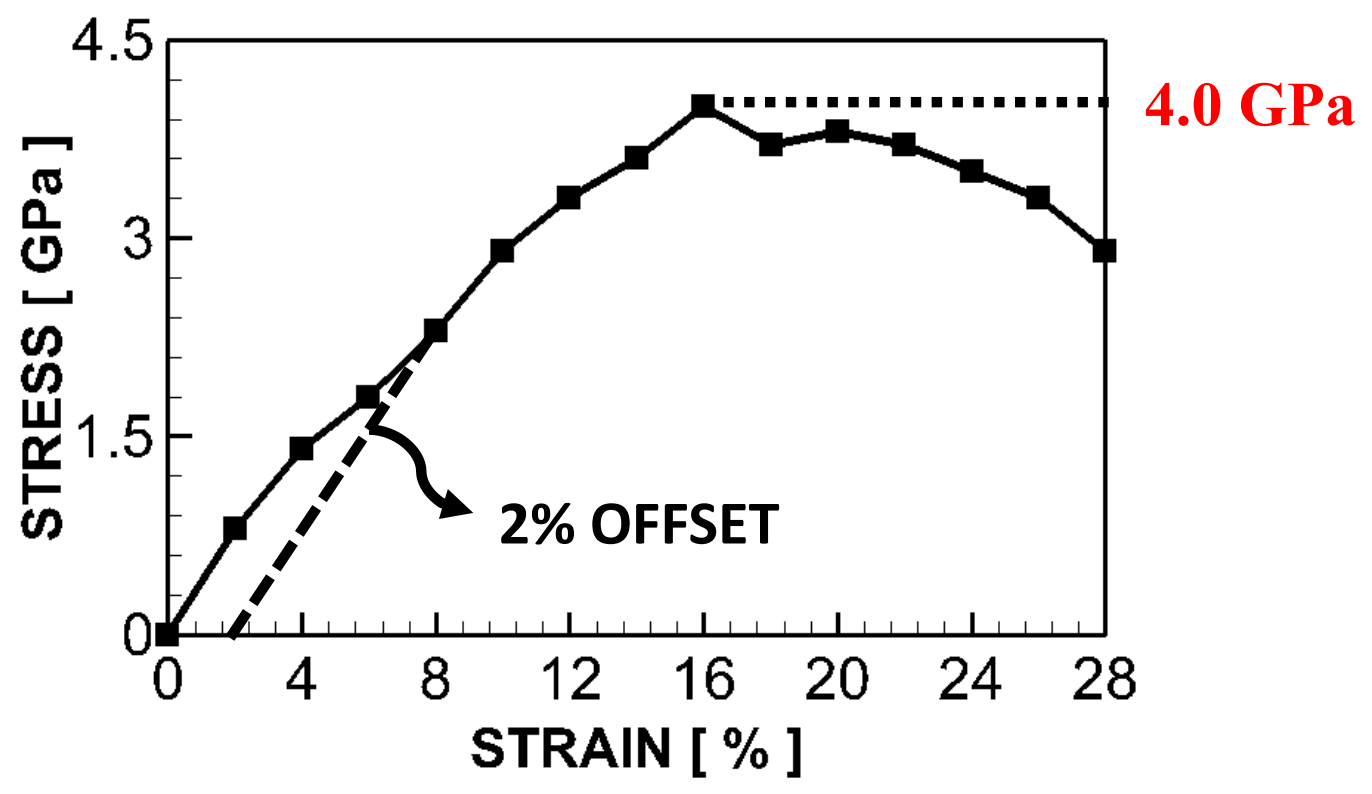

Figure 25: Stress-strain plot of pure W (GB thickness=0nm)

In the next figure, the stress-strain relations of unsaturated cases are plotted. For the unsaturated Ni-doped $\mathrm{W}$, the yield stress is also observed at $8 \%$ strain. At the strain of $12 \%$ and $18 \%$, the curves have two main peaks, which can be considered as the maximum tensile strength. The yield stress and these two peak stresses are plotted in (b) for clear observation. While the maximum tensile strength does not change by the variation of the $\mathrm{Ni}$ volume fraction the yield stress is reversely dependent on the Ni-volume fraction. This implies that the Ni does not reinforce the bonding strength when the GB of W is in the form of unsaturated (GB thickness $=0.3 \mathrm{~nm}$ ), but degrading the yield stress of the structure. 


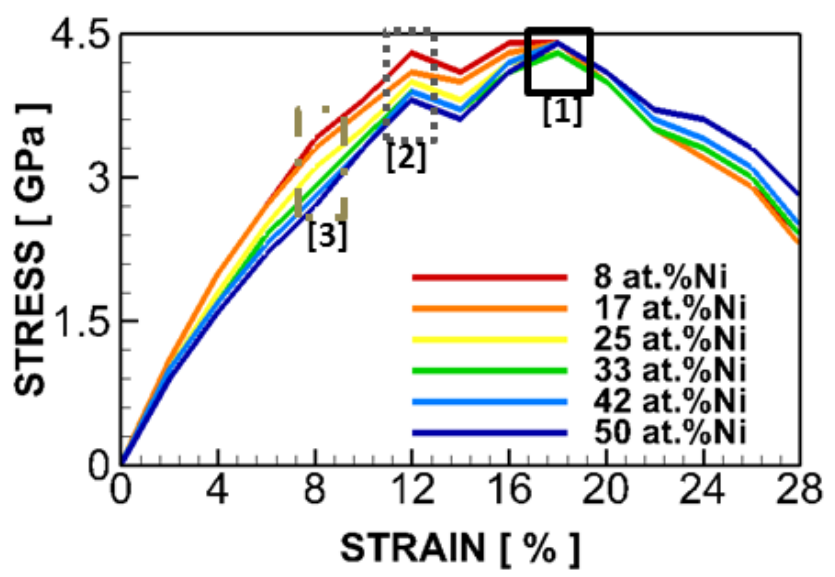

(a)

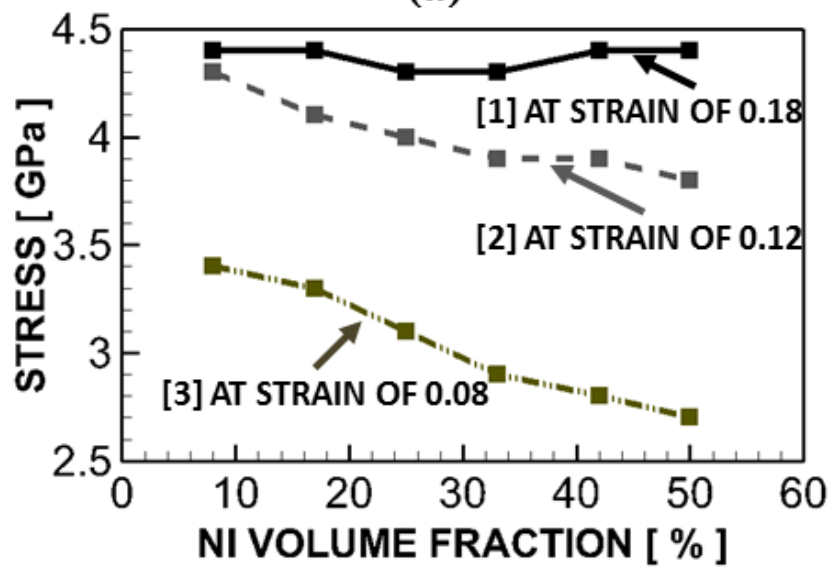

(b)

Figure 26: (a) Stress-strain relation of unsaturated Ni-doped W (GB thickness $=0.3 \mathrm{~nm}$ ), (b) comparison of yield stress and peaks of the curves.

Unlike the unsaturated case, the stress-strain curves of saturated Ni-doped W show strengthening of the material by the addition of Ni in the GB as shown in the figure below. While the yield stress is still observed at $8 \%$ strain the first peak appears at $16 \%$ strain, which is higher than $12 \%$ in the unsaturated case. From this point, the plastic deformation initiate earlier in the unsaturated tungsten than the saturated tungsten. The stress-strain curves of 7 different Ni volume fractions can be divided into two types. While the curves of $25 \% \sim 41 \% \mathrm{Ni}$ volume fraction decrease after the first peak at 16\% strain, the curves of 50\% 75\% increase even after the first peak. This feature distinguishes the specific boundary of $\mathrm{Ni}$ amount that changes the mechanical properties of saturated $\mathrm{W}$. While the overall tendency is that the larger amount of Ni increases the maximum tensile strength the highest value of tensile strength is observed in the case of $58 \% \mathrm{Ni}$ volume fraction. 


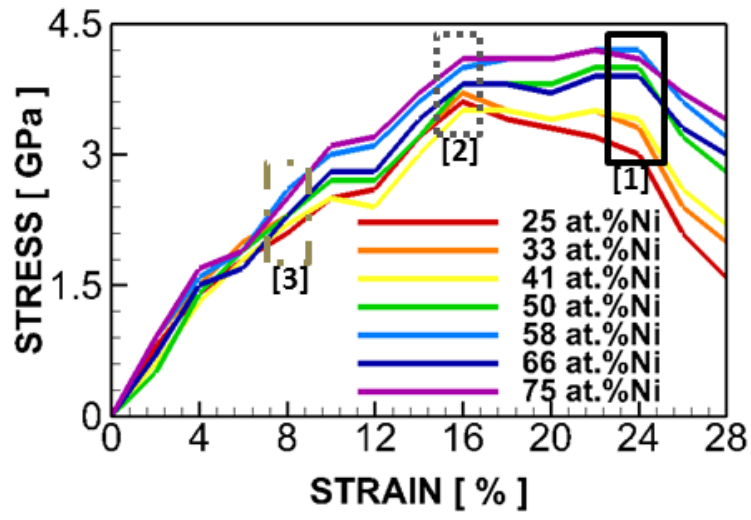

(a)

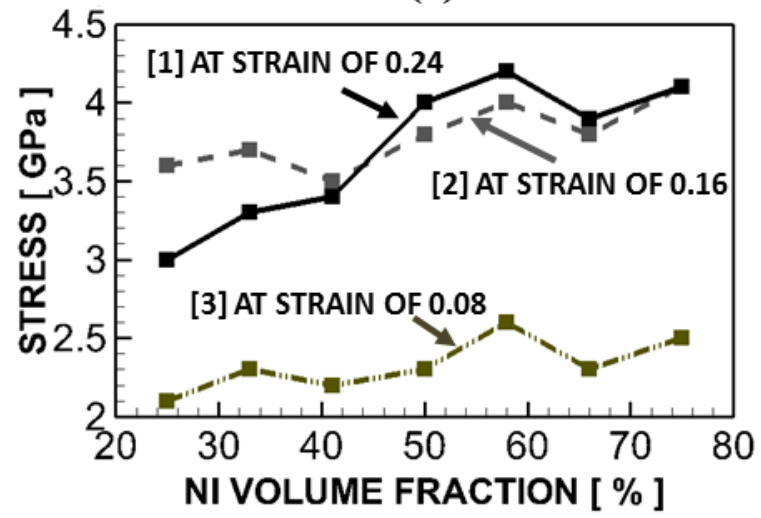

(b)

Figure 27: (a) Stress-strain relation of saturated Ni-doped W (GB thickness=0.6nm), (b) comparison of yield stress and peaks of the curves.

Strengthening of an atomic structure can be restates that those have higher bonding force, which comes from the activity of electrons of the neighboring atoms. In order to distinguish which part of electron densification contributes for having higher bonding force, the DOS is taken for the comparison as shown in the figure below 


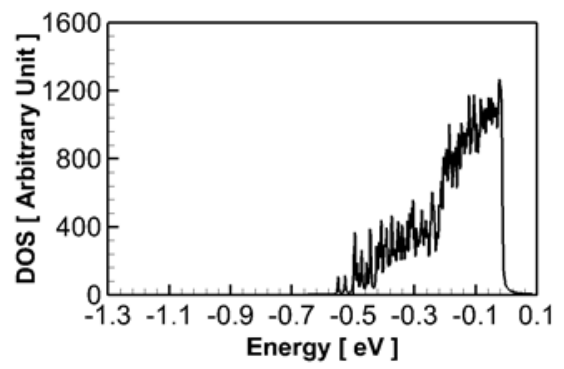

(a)
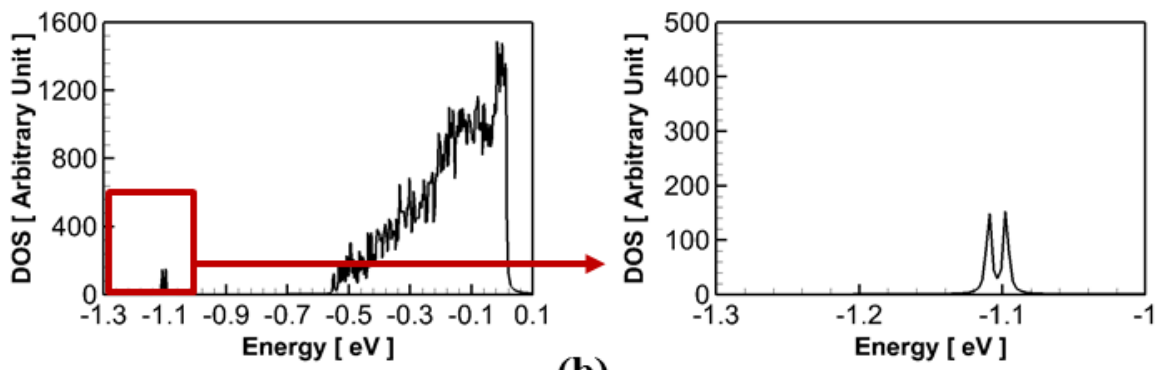

(b)
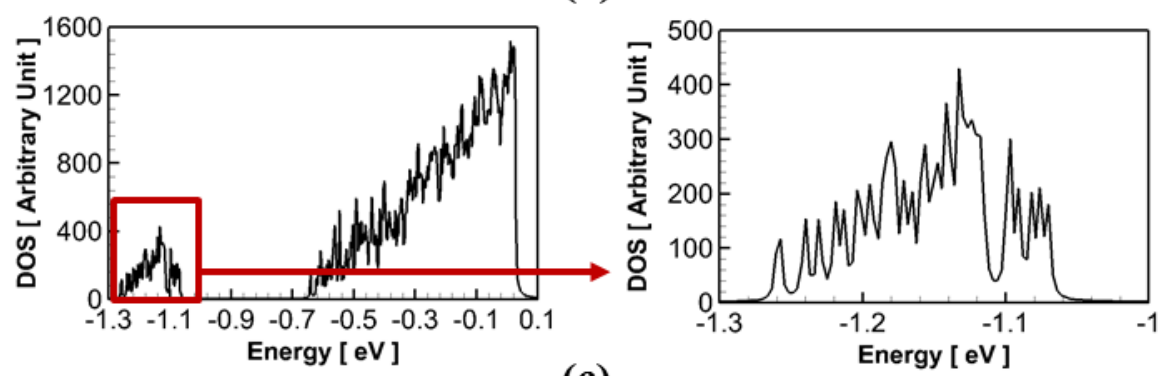

(c)

Figure 28: Density of States plots of (a) pure W, (b) unsaturated Ni-doped W with $8 \% \mathrm{Ni}$ volume fraction, along with the detail view of peaks at the range of $-1.3 \sim-1.0$, and (c) saturated $\mathrm{Ni}$-doped $\mathrm{W}$ with $75 \% \mathrm{Ni}$ volume fraction, along with the detail view of peaks at the range of -1.3 $\sim-1.0$

Figure 28 (a), (b), (c) are DOS plots of electrons in f-orbital for the pure W, unsaturated $\mathrm{Ni}$-dope $\mathrm{W}$ with 8 at. $\% \mathrm{Ni}$, and saturated $\mathrm{Ni}$-doped $\mathrm{W}$ with 8 at.\% $\mathrm{Ni}$, respectively. The reason of selecting f-orbital for the investigation is that only f-orbital is showing distinctive changes in lower energy level, which perhaps directly influence the bonging strength of the atomic structure. Distinctive feature among these plots are the changes of DOS in the energy range of -1.3 -1.0. While the pure $\mathrm{W}$ case does not have any activity in this range, the addition of Ni to the $\mathrm{W}$ GB significantly increase the number and the magnitude of peaks in the energy range of -1.3 -1.0. Specific activities of electrons in this range are quantitatively examined by summing the DOS between $-1.3 \sim-1.0$ and plotted with corresponding electron density map as shown in the figure below. 


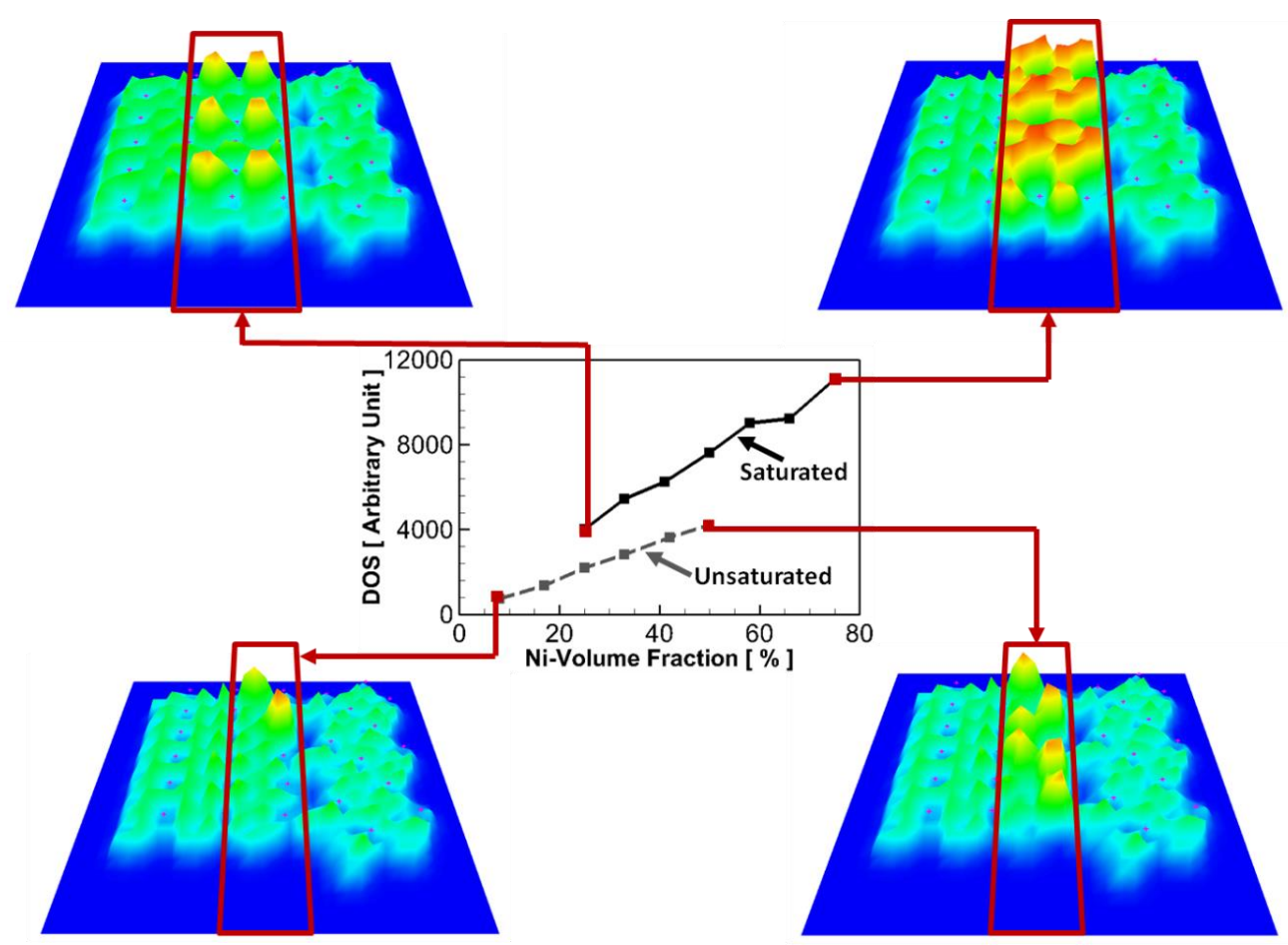

Figure 29: Electron density plots corresponding to the density of states as a function of Ni-volume fraction.

The electron density is illustrated by contours which have scaled range of $0 \sim 1$. The electron density contour is cross section of $\mathrm{x}-\mathrm{y}$ plane in the mid-point. As the amount of Ni-addition increases the high electron density region becomes larger.

A relation is constructed based on the ratio between the pure tungsten's UTS, $T_{\text {pure }}$ and obtained maximum tensile strength, $T_{\max }$ can be compared with the ratio of surface energy and cohesive energy, which can be converted from the internal energy of the atomic structure. By applying the factors of variation such as the temperature $t$ and nickel volume fraction $n$ to form a function, the balanced relation can be developed in $f(t, n)$. Also, the thickness $w$ of the nickel added GB need to be considered since the internal energy within the atomic system can be varied by the number of atom which interact as the strains are applied. The function of GB thickness is denoted as $\mathrm{g}(w)$.

$$
\frac{\mathrm{T}_{\max }}{\mathrm{T}_{\text {pure }}}=\cdot \frac{C E}{C D} \cdot \frac{1}{\Phi} \cdot f(t, n) \cdot g(w)
$$

Here, CE is the cohesive energy, $C D$ is the crack density, and $\Phi$ is the surface energy of tungsten. The surface energy of the tungsten is known to be $2.49 \mathrm{~J} / \mathrm{m}^{2}{ }^{14}$. The crack density is 1.08 $\mathrm{nm}^{2}$, which is measured cross sectional area of the atomic structure. The cohesive energy, which is internal energy in the atomic calculation, is obtained by measuring the area under the curve of the stress-strain plot of each simulation. The function in terms of the temperature and the nickel volume fraction is taken to numerical analysis to fit into the actual simulation data. When comparing the ratio of tensile strength with that of energy terms the multiplication of the surface 
energy with crack density gives the theoretical energy that be generated while the tension is acted on the atomic structure. For the function $f(t, n)$, which is in terms of the temperature and the nickel volume fraction, temperature is scaled by the tungsten's melting point $T_{\text {melt }}$ and the nickel volume fraction is multiplied by a coefficient to give nickel volume fraction dependence and temperature dependence to the formula. The function of GB thickness $\mathrm{g}(w)$ is standardized by scaling with tungsten atomic lattice.

$$
\mathrm{T}_{\max }=\mathrm{T}_{\text {ideal }} \cdot \frac{C E}{C D} \cdot \frac{1}{\Phi} \cdot\left(e^{n}-0.02 \cdot \frac{t}{t_{\text {melt }}}+3.4\right) \cdot\left(\frac{1.6 \cdot a-0.032 \cdot w}{a}\right)
$$

The nickel volume fraction appears to play a role in improving the tensile strength of tungsten especially in the case of saturated tungsten while the effect of nickel addition in unsaturated tungsten is lesser. The developed relation can be used to predict the maximum tensile strength of nickel-doped tungsten, which contains nickel addition in different thickness of GB, both for unsaturated and saturated tungsten. The qualitative improvement in the tensile strength as a function of nickel volume fraction for different GB thickness is compared with the proposed relation in the figure below.

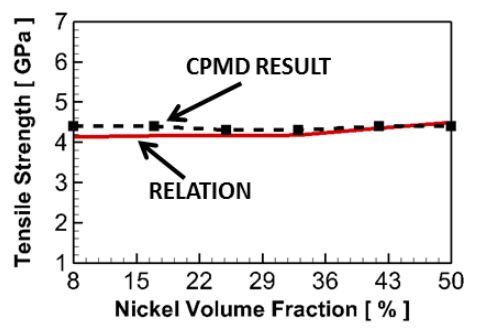

(a)

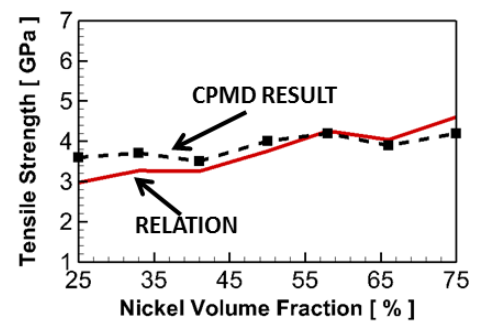

(b)

Figure 30: Comparisons of the GB strength as a function of volume fraction with analytical result derived from the proposed relation for (a) unsaturated Ni-doped W (GB thickness $=0.3 \mathrm{~nm}$ ), and (b) saturated Ni-doped W (GB thickness $=0.6 \mathrm{~nm}$ )

The highest peaks of electron density are observed in the region of $\mathrm{Ni}$ substitution. As $\mathrm{Ni}$ volume fraction increases the electron density increases and the increase of DOS of f-orbital in the specific energy range is due to this aspect. Since we have observed the dependence of DOS of f-orbital to the bond strength of the atomic structure, the verification of this dependency is necessary. From the sum of DOS, the unsaturated case shows linearity while the saturated case shows some fluctuations. Based on the linear function that is fitted along the unsaturated case, the sum of DOS was rescaled. Then, the maximum tensile stress of both unsaturated and saturated cases is compared with the rescaled DOS of f-orbital in the following figure for the verification of dependency. 


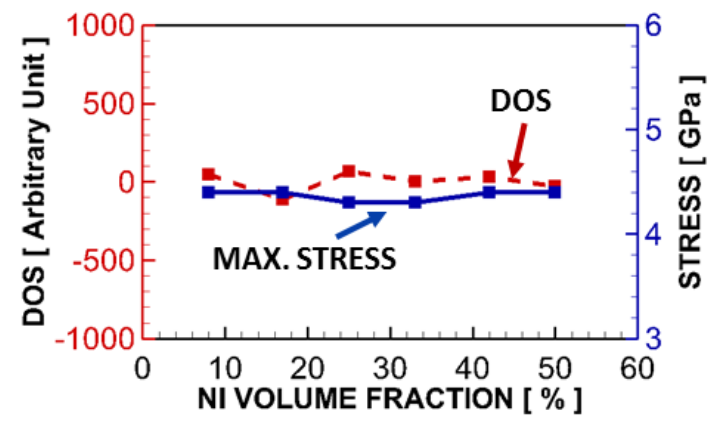

(a)

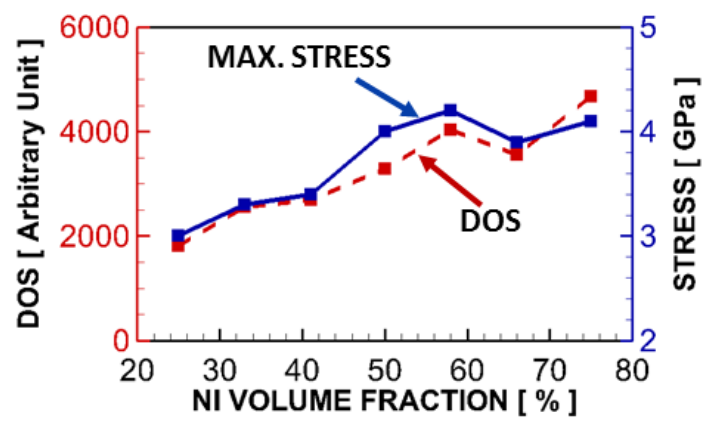

(b)

Figure 31: Comparison of the maximum tensile stress with the density of states (sum in the range of $-1.3 \sim-1.0 \mathrm{eV}$ ) for f-orbital of (a) unsaturated case (b) saturated case

While there are other factors which could influence the bond strength the DOS of f-orbital in the specific energy region $(-1.3 \sim-1.0 \mathrm{eV})$ became to be one of the important factors for predicting the maximum tensile stress of Ni-doped W. If the DOS was to define the effect of electrons, another observation could come from the phonon, which reflects the vibration of atoms in the GB structure. The phonon dispersion curves are shown in the figure below for different structures: no GB (GB thickness $=0 \mathrm{~nm})$, unsaturated (GB thickness $=0.3 \mathrm{~nm})$, and saturated (GB thickness $=0.6 \mathrm{~nm})$. First two transverse $(\mathrm{T})$ and one longitudinal $(\mathrm{L})$ components are displayed as a function of wave vectors. While the phonon dispersion curves are observed on the positive side of the plot, small portion of the transverse curve in unsaturated case has negative frequency in the direction of $[\xi \xi \xi]$. The main reason of negative phonon frequency is known to be lattice instabilities in the direction ${ }^{15}$. Lattice instability in the direction of $[\xi \xi \xi]$ indicates that as the tensional loading is applied the atomic structure of specific region is acting as a defect which could result the reduction of the strength. This implies that the less saturated Ni-doped $\mathrm{W}$ is structurally imperfect comparing to that of more saturated case. 


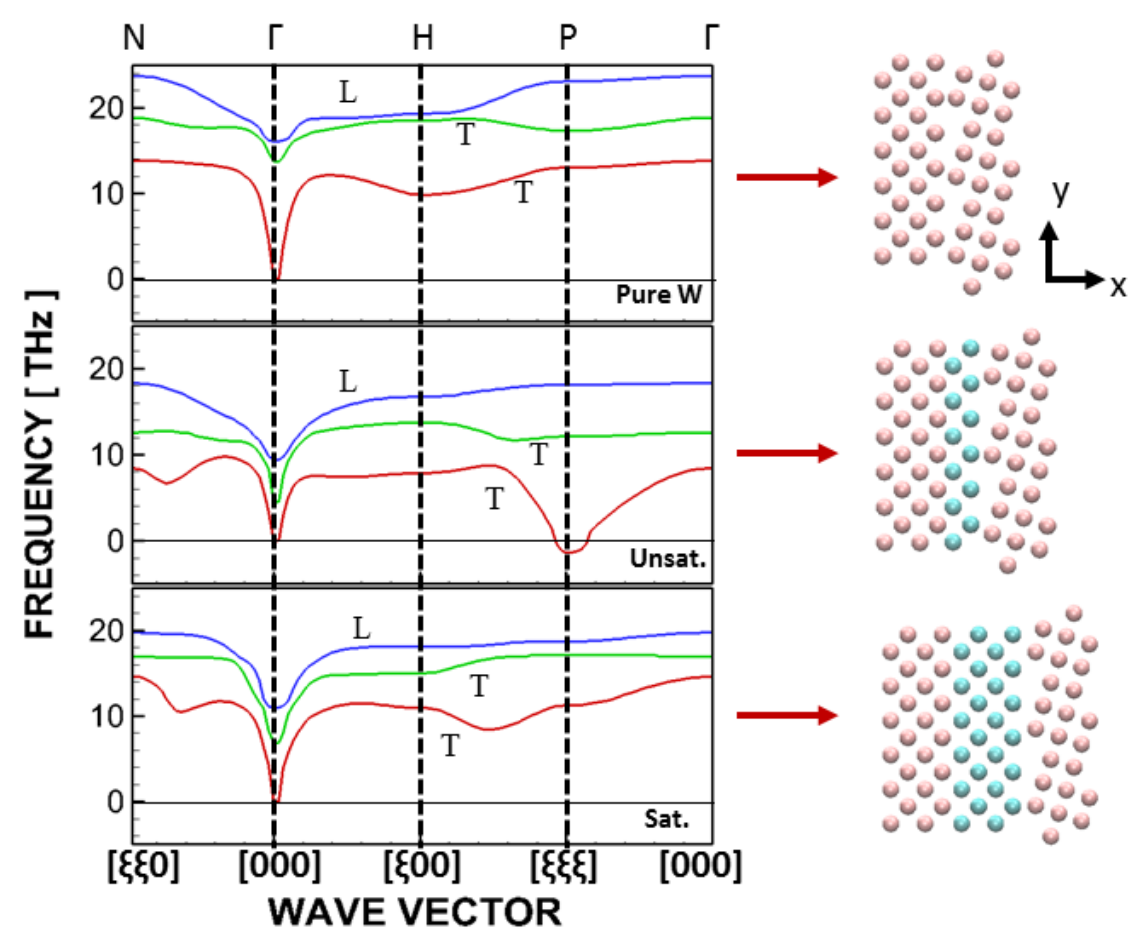

Figure 32: Phonon dispersion of the base $\mathrm{W}$ structures, which are cases of no GB (GB thickness $=0 \mathrm{~nm})$, unsaturated $\mathrm{W}(\mathrm{GB}$ thickness $=0.3 \mathrm{~nm})$, and saturated $\mathrm{W}(\mathrm{GB}$ thickness $=0.6 \mathrm{~nm})$

While the obtained phonon dispersion relation contains several degrees of freedom for atoms in the structure, the 1st transverse component for the direction of [ $\xi 00]$ and [ $\xi \xi 0]$ are taken for the comparison in Fig. 49. It is important to note that the pure W model has idealistic atomic structure based on the assumption of zero thickness GB, which obviously provide the most stabilized form of the structure. Excluding the case of no GB, more saturated form (greater GB thickness) gives higher frequency which is related to the higher bond strength along the given directions. The Fig. 34 shows that the atomic structure of pure $\mathrm{W}$ case provide higher phonon frequency in overall. Despite the level of phonon frequency, the stress-strain curves in Figure 282930 tells us that for those with structure set with any saturation level (GB thickness larger than 0 ), substitution of $\mathrm{Ni}$ atoms with $\mathrm{W}$ gives higher tensile strength. From these relations, we could see that the substitution of $\mathrm{Ni}$ atoms with $\mathrm{W}$ atoms plays role of compensating enough for the low frequency of phonon. 


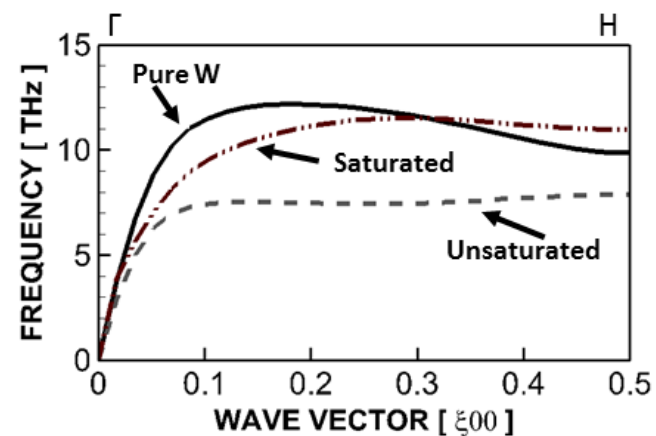

(a)

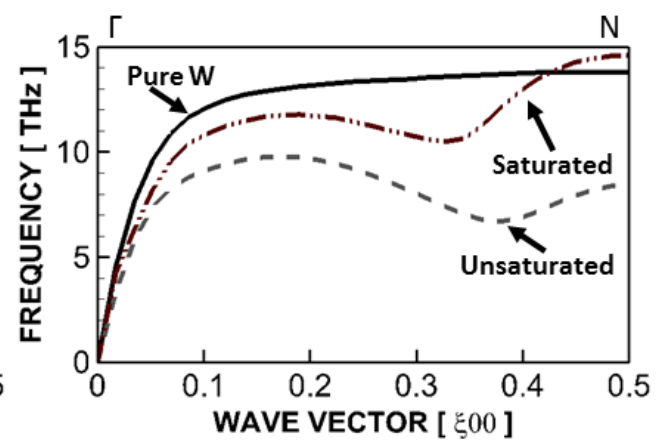

(b)

Figure 33: Phonon dispersion of different interface structure with respect to the direction of (a) $\mathrm{x}$-axis $[\xi 00]$ and (b) diagonal $[\xi \xi 0]$

\section{§5. Investigation on the Role of Grain Boundary in the Fracture using 3-D Extended Finite Element Method}

The extended finite element method (XFEM) was developed in 1999 by Ted Belyschko and collaborators $^{16}$. The XFEM was developed to ease difficulties in solving problems with localized features that are not efficiently resolved by mesh refinement. One of the initial applications was the modeling of fracture in a material. Discontinuous basis functions are added to standard polynomial basis functions for nodes that belonged to elements that are intersected by a crack to provide a basis that included crack opening displacements. A key advantage of XFEM is that in such problems the finite element mesh does not need to be updated to track the crack path.

In this research, XFEM is implemented to perform a numerical simulation on three dimensional polycrystalline microstructures of silicon carbide and tungsten. For performing the simulation, a commercial program, ABAQUS v6.10 was used. Three grain typeswere used to model a polycrystalline microstructure as representations of different orientation ${ }^{17}$. Similarly, three different types of grain are assumed in this study. Due to the limitation on designing complex 3-D geometry in ABAQUS program, simple cubic shaped grains are used to model a 3-D polycrystalline microstructure, which is used for a test simulation. Model of simple cubic shaped geometry can be modified into realistic polycrystalline microstructure by editing node coordinates and connectivity data in the ABAQUS input file. Such procedure is described in the figure below

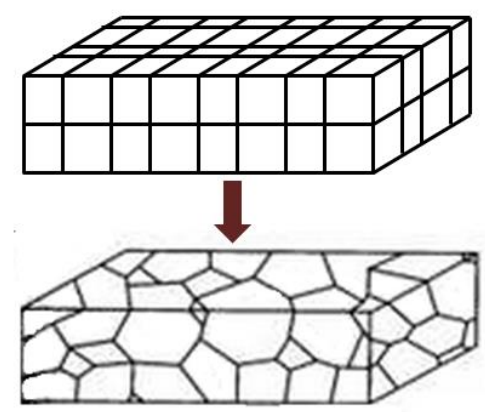

Figure 34: Modification of microstructural geometry 
Each of three different types of grains are assigned into each cubic element and placed as shown in Figure 34. Three sets of properties have variation between 200GPa 400GPa for the elastic modulus and 200MPa 400MPa for the maximum fracture strength. Poisson's ratio is 0.3 for all three types. Property variation is exaggerated in order to observe distinct result to verify property effect on the crack propagation direction. Each type of cubic is placed one after another so that the same type of cubic grain is not placed together. Tensile loads are applied from vertical direction and initial crack is also implemented in the model.

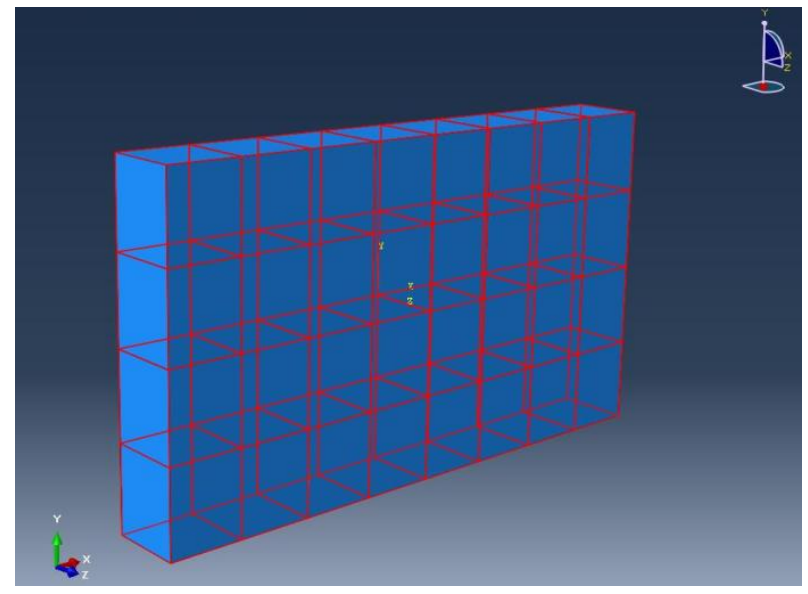

Figure 35: Simple cubic grains

The simulation has gone through static as a step and recorded the crack path along with von Mises stress. The result showed an interesting crack path. Kinked crack path was observed due to the heterogeneous property within the microstructure.

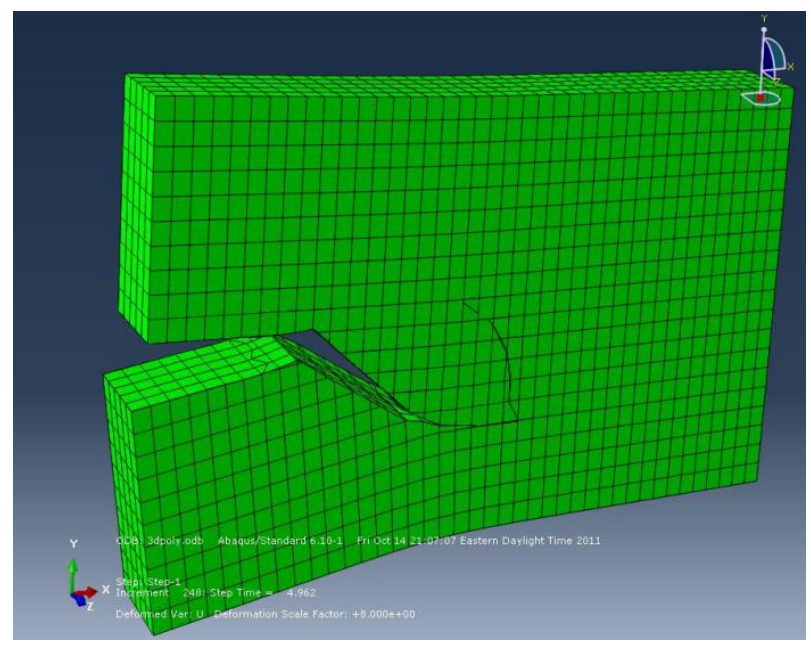

Figure 36: Crack path of 3D XFEM simulation 


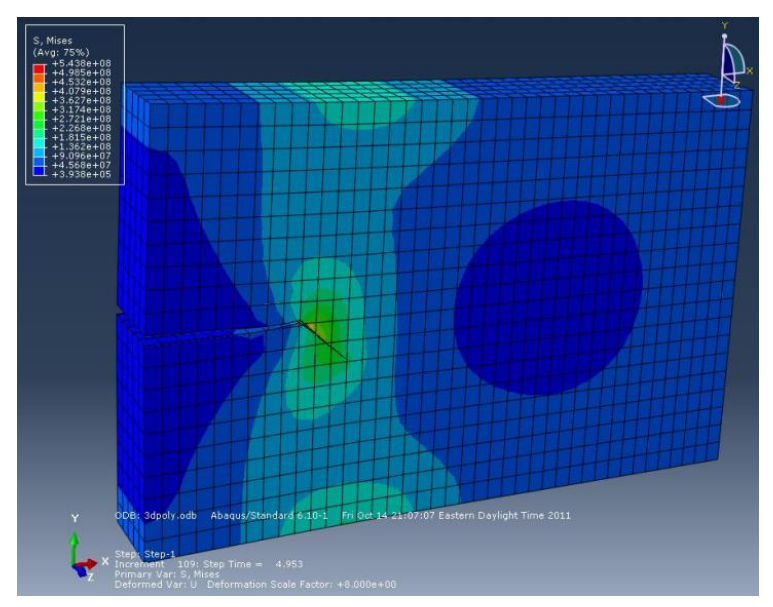

(a)

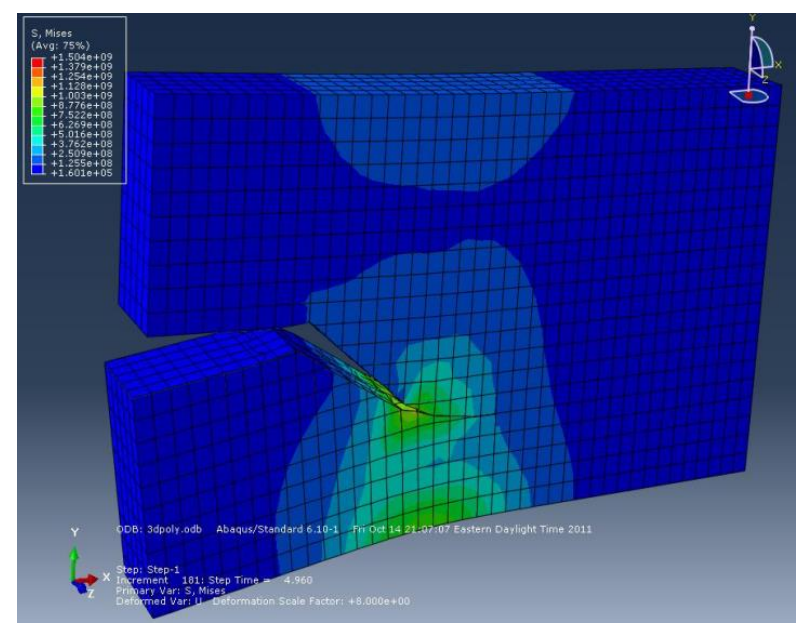

(b)

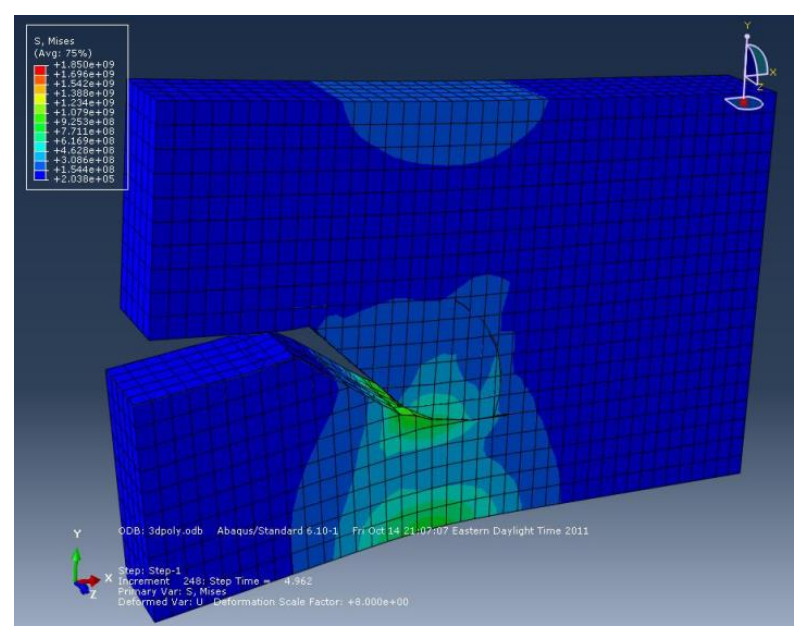

(c)

Figure 37: Crack propagation with von Mises stress contour of (a) initial stage, (b) middle stage, and (c) end stage. 
Von Mises stress contours of three stages are shown in figure 37. Due to the exaggerated property setup which was described earlier a great kinking angle of crack path is clearly observed. Now the test run of 3D XFEM simulation is completed and detail modeling of realistic polycrystalline microstructure is in the current progress. MATLAB is being used to modify node coordinates and connectivity of the ABAQUS input file. HYPERMESH also is considered to simplify this procedure. After the structure modeling, additional phase will be included after the input file modification for representing grain boundaries. By including grain boundary an investigation of the effect of grain boundary to the fracture behavior can be conducted. The interfacial properties will be decided based on the quantum simulation result.

The extended finite element method (XFEM) uses the partition of unity framework to model strong and weak discontinuities independent of the finite element mesh. This allows discontinuous functions to be implemented into a traditional finite element framework through the use of enrichment functions and additional degrees of freedom.

$$
u^{h}(x)=\sum_{I} N_{I}(x) u_{I}+\sum_{I} N_{I}(x) v(x) a_{I}
$$

Enriched nodes for crack inside a plane can be described as shown in the figure below.

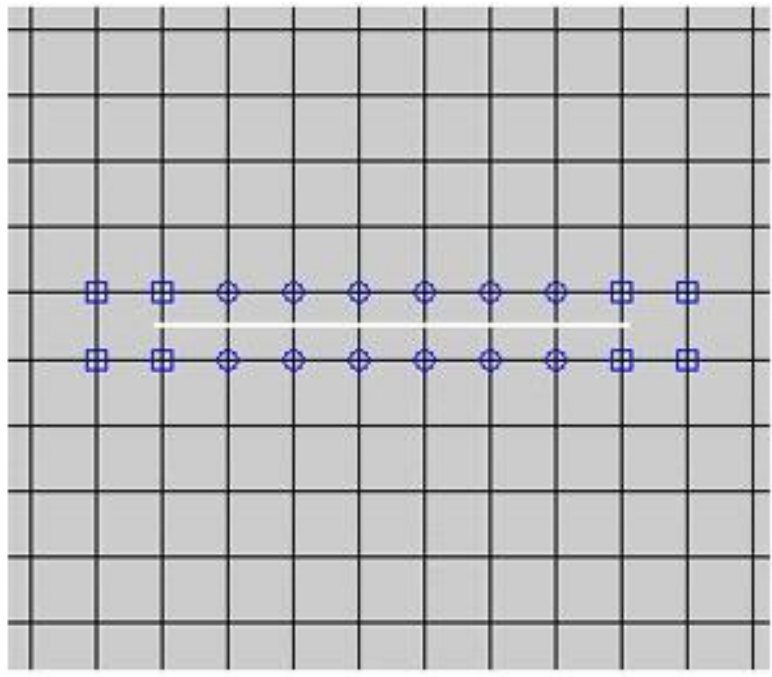

Figure 38: Schematic illustration of enriched nodes for crack

As the nodal displacement is now a function of both the traditional and enriched degrees of freedom it is common practice to shift the enrichment functions such that the enrichment functions vanish at the nodes.

$$
\gamma_{I}(x)=v(x)-v_{I}(x)
$$

Therefore the XFEM approximation with the shifted enrichment function takes the following form. 


$$
u^{h}(x)=\sum_{I} N_{I}(x) u_{I}+\sum_{I} N_{I}(x) \gamma_{I}(x) a_{I}
$$

Cracks are modeled using a combination of two enrichment functions. One for the complicated behavior at the crack tip and a Heaviside step function to represent the discontinuity across the body of the crack.

$$
v_{a}(x)=\left[\sqrt{r} \sin \frac{\theta}{2}, \sqrt{r} \cos \frac{\theta}{2}, \sqrt{r} \sin \theta \sin \frac{\theta}{2}, \sqrt{r} \sin \theta \cos \frac{\theta}{2}\right]
$$

Moes introduced a modified absolute value enrichment of the following form where zeta is the value of the level set function at node $I$. Note that this function vanishes at the nodes itself and therefore does not need to be shifted as is typical of other enrichment functions.

$$
v(x)=\sum_{I} N_{I}\left|\zeta_{I}\right|-\left|\sum_{I} N_{I} \zeta_{I}\right|
$$

The enrichment function for a void takes a different form from the traditional enrichment. Instead a step function is used such that $\mathrm{V}(\mathrm{x})$ takes a value of 1 in the domain and 0 inside the void.

$$
u^{h}(x)=V(x) \sum_{I} N_{I}(x) u_{I}
$$

In order to apply this method to 3-dimensional microstructure for fracture analysis, developing of model is the one of the most important procedure for accurate result.

A software that has capable of generating random Voronoi tessellation was used to develop a three-dimensional polycrystalline model which can be a base model for our fracture analysis. Dimension of the proposed model is described.
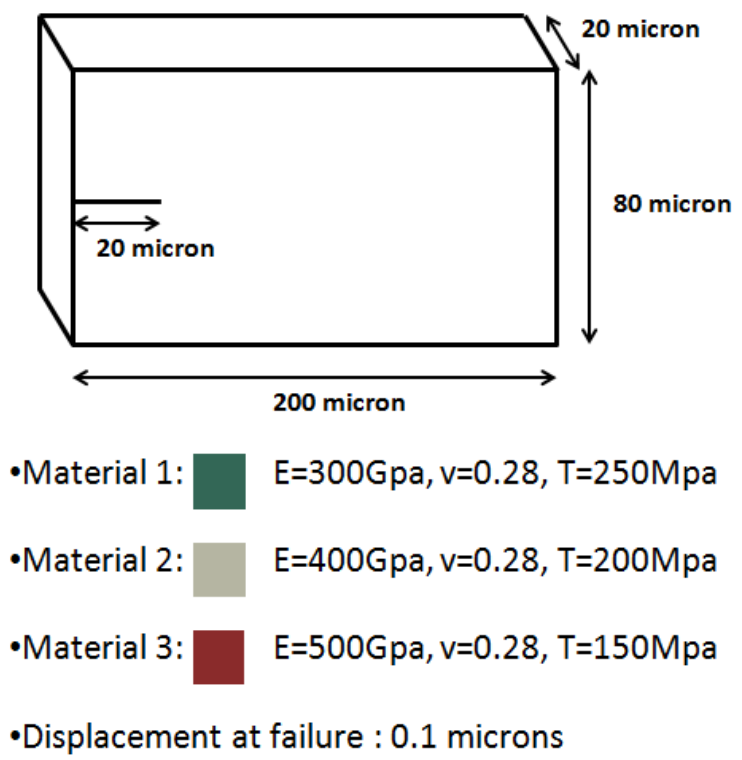

Figure 39: Dimension of the model 
Using the above dimension, a polycrystalline model is generated. Basic structure of the grains is shown in the figure below. Then, the grains of the structure are assigned with 3 types of material properties for investigation of polycrystalline fracture behavior including the pattern of the crack. The meshed model is shown.

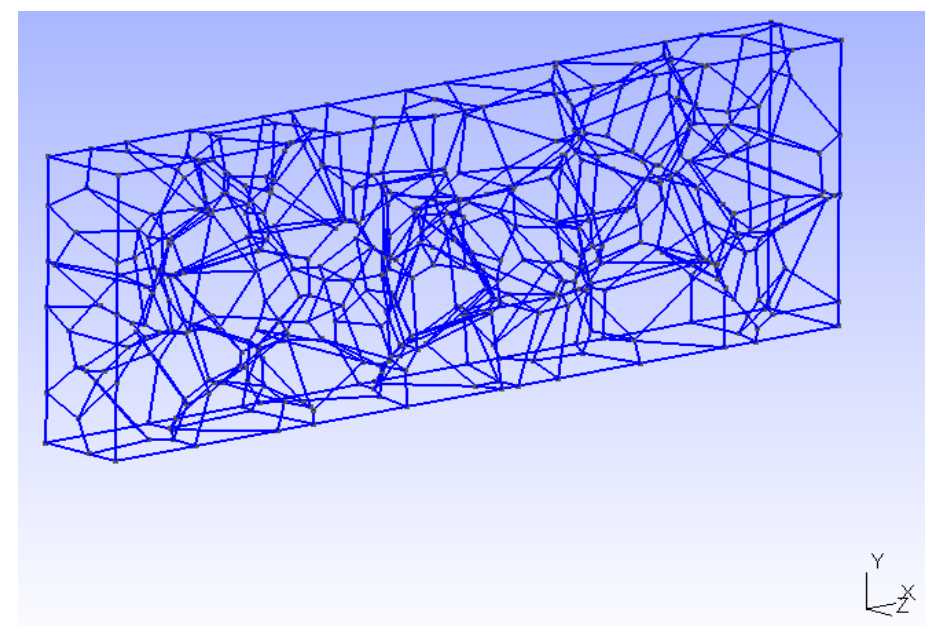

Figure 40: Structural frames of the grains in the model

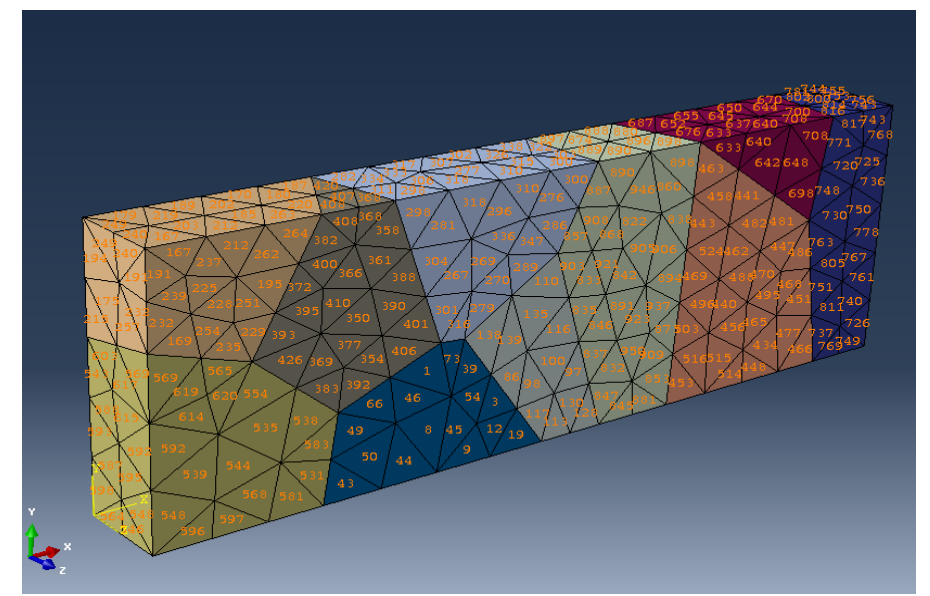

Figure 41: Meshed model with each element numbered

An initial crack of 20 micron is added to the model and the failure is designed to be created at the displacement of 0.1 micron. From the meshed model, boundary conditions are implemented into the model. Uniformly distributed pressure load of $10000 \mathrm{~N} / \mathrm{mm}^{\wedge} 2$ is applied to the upper and lower surfaces.

Meshed model is assigned with unique number for each element. This is for the purpose of assigning the corresponding material properties to the right element based on the geometric coordinates. After assigning the material properties for each phases and implementing the boundary conditions, the model before the simulation is shown in the figure below. 


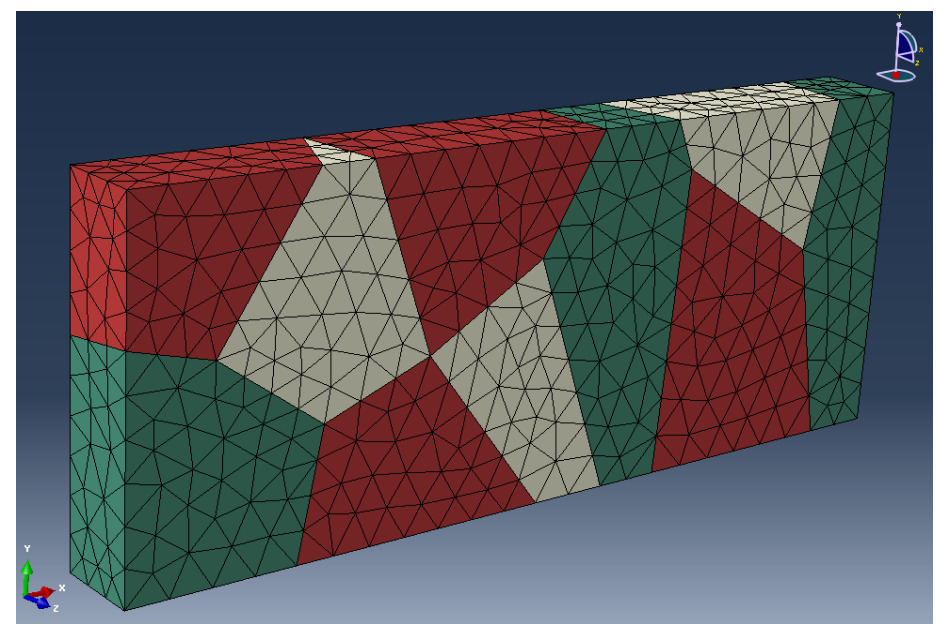

Figure 42: Model with the properties applied to each elements belonging to their phase types

The model has been undergone the computing process and the crack path and overall structure plot is obtained as shown.

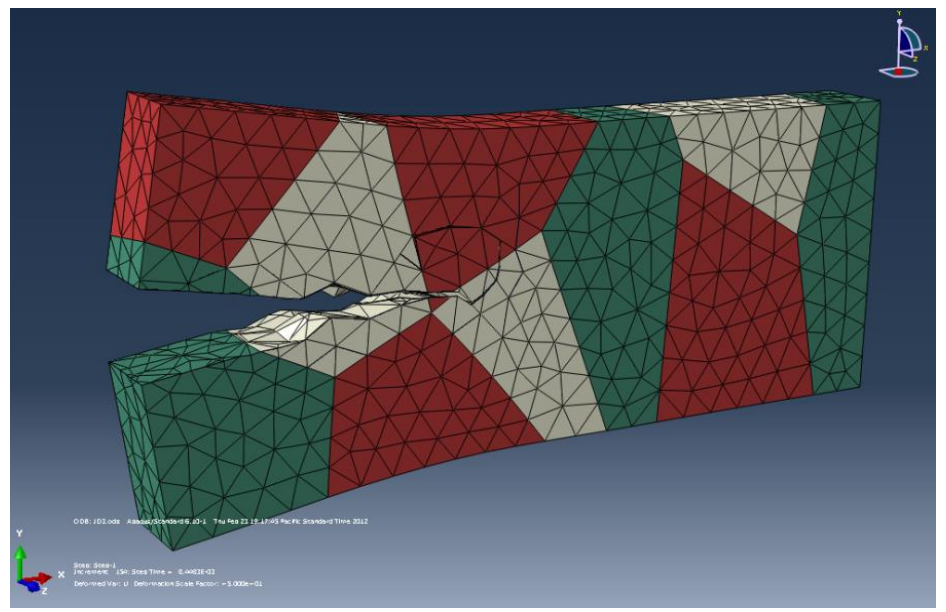

Figure 43: Plot of the crack propagation during the XFEM simulation

Due to the complexity of element shape, the pattern of the crack propagation is shown to be also complex. To find the role of the element size to the crack propagation path, convergence study with variation of element size was conducted. Giving the variations to the element size, four different simulation sets with different number of elements were prepared for the convergence study. The effect of element size to the crack propagation path was significant and the results are shown in the figure below. 


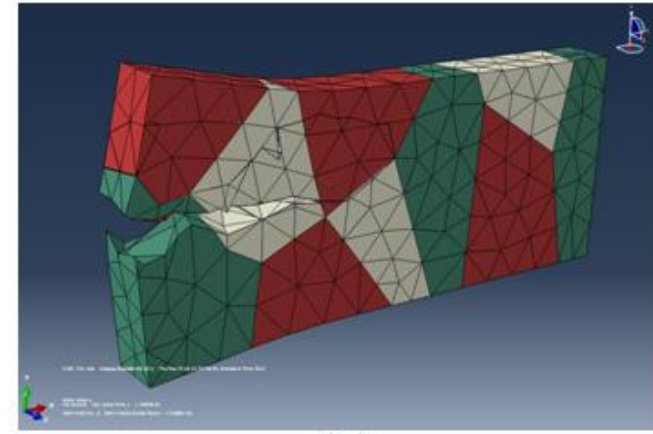

(a)

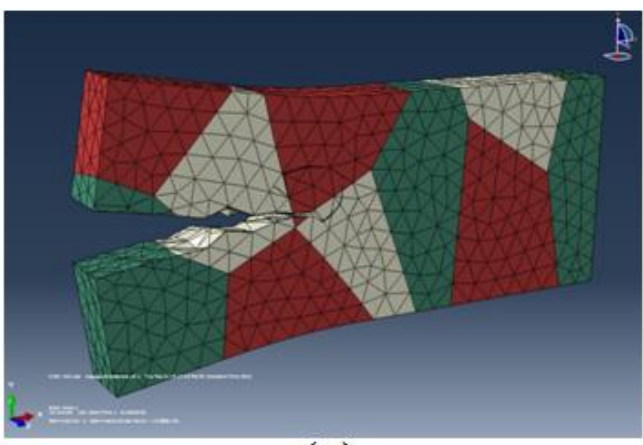

(c)

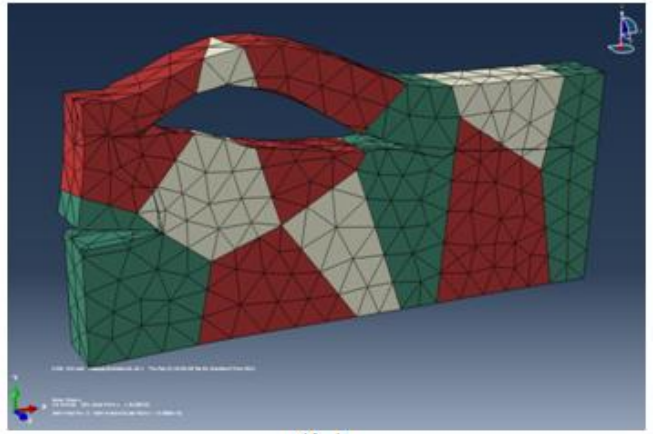

(b)

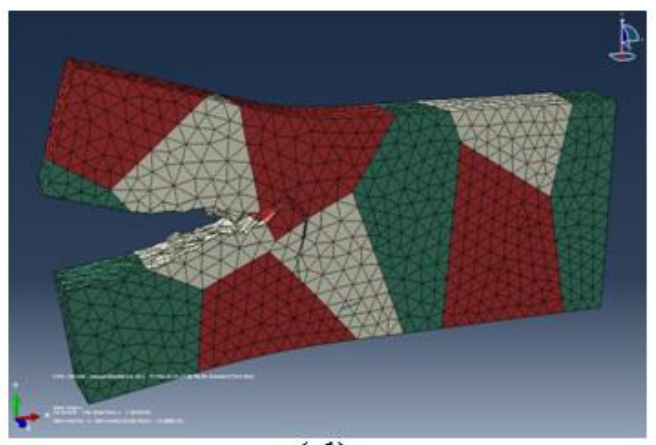

(d)

Figure 44: Crack propagation paths with variation of element size

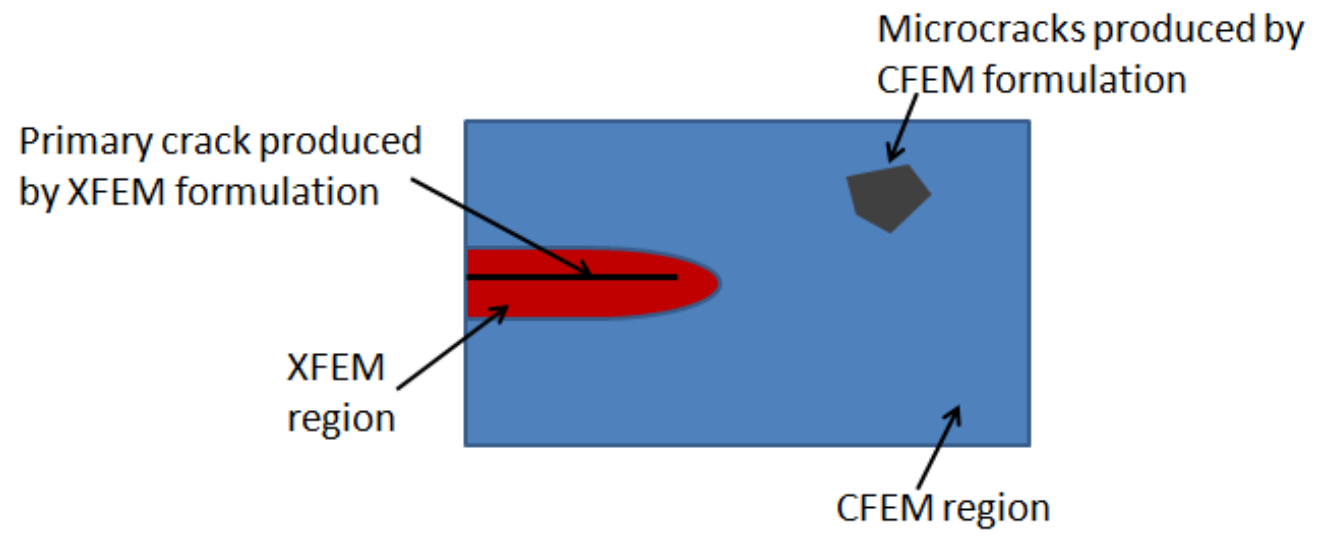

Figure 45: Schematic description of proposed method

Microstructure of polycrystalline tungsten is observed to examine the GB region specifically. While the thickness of GB in saturated tungsten is found to be approximately $0.6 \mathrm{~nm}$, we have used quantum models to find the fracture properties of GB region of tungsten material. 


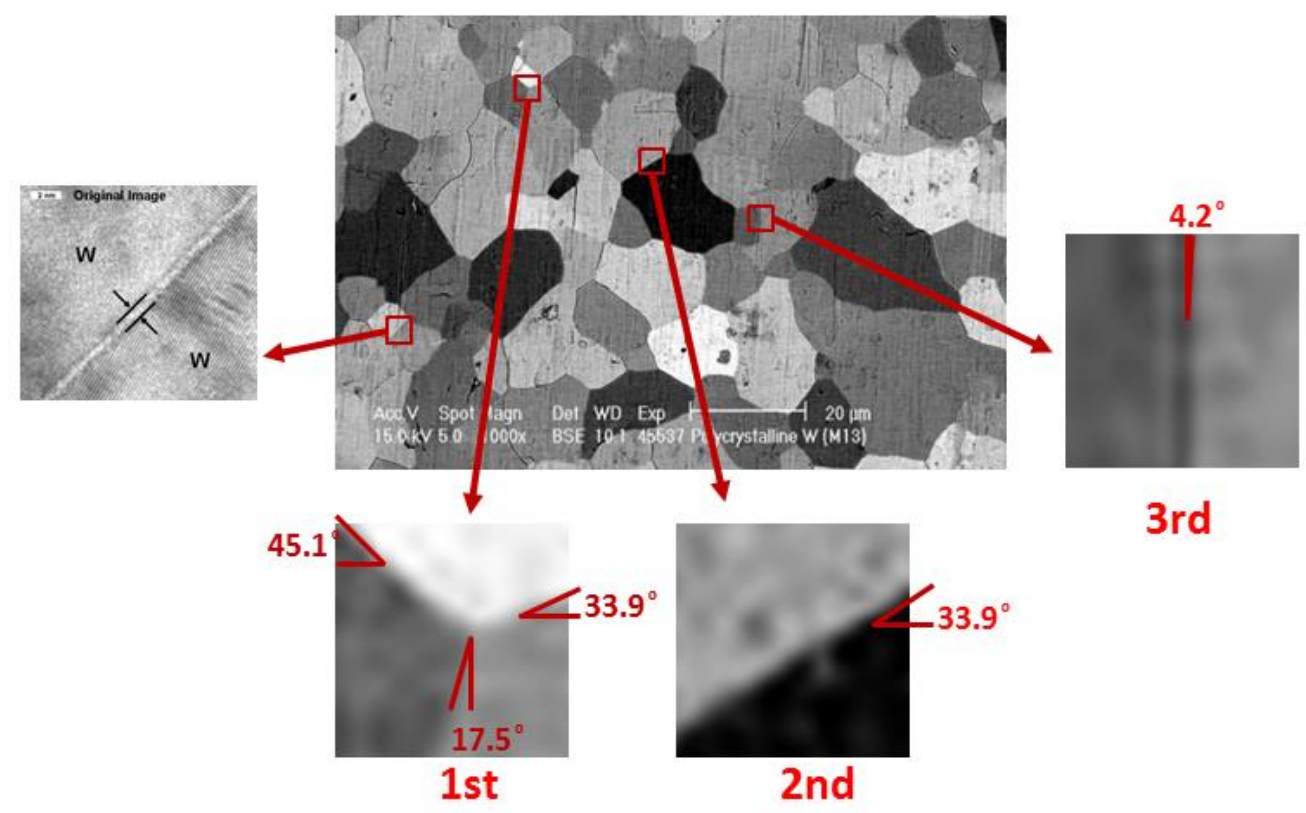

Figure 46: Image of polysrystalline tungsten with three selections of typical GB regions

The main three GB regions are constructed in 3-dimensional models which includes multiple phases. While carefully predicting that the angle of GB layout can affect the angle of crack propagation, developed model is exactly representing the geometry of the real images. Using the XFEM feature in ABAQUS, the weak form can be described as

$$
\delta W^{\mathrm{int}}=\delta W^{\mathrm{ext}}+\delta W^{\mathrm{coh}}
$$

where the internal work term is equal to summation of external work and the work done by cohesive. The work done in internal, external, and cohesive is as below.

$$
\left\{\begin{array}{l}
\delta W^{\mathrm{int}}=\int_{\Omega} \frac{\partial \delta \mathbf{u}}{\partial \mathbf{x}}: \sigma \mathrm{d} \Omega \\
\delta W^{\mathrm{ext}}=\lambda \int_{\Gamma_{1}} \delta u \cdot \tau_{0} \mathrm{~d} \Gamma \\
\delta W^{\mathrm{coh}}=\int_{\Gamma_{c+}} \delta \mathbf{u}^{+} \cdot \tau^{c+} \mathrm{d} \Gamma+\int_{\Gamma_{c-}} \delta \mathbf{u}^{-} \cdot \tau^{c-} \mathrm{d} \Gamma
\end{array}\right.
$$




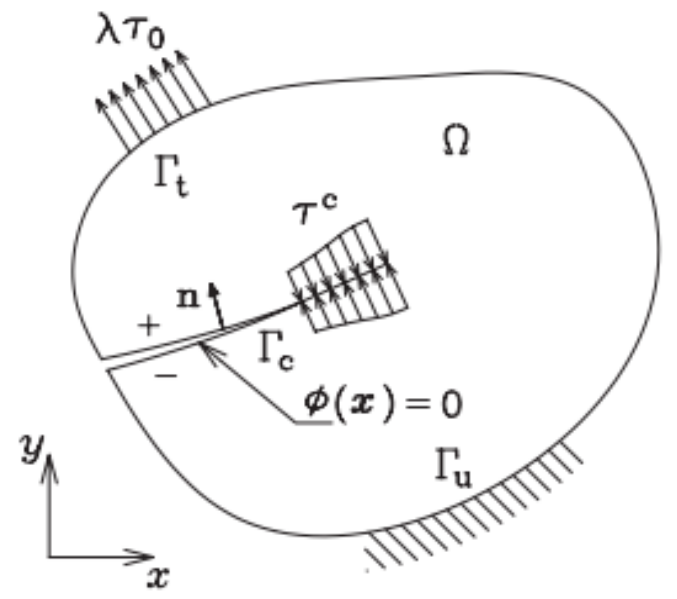

Figure 47: Schematic illustration of boundary conditions and traction applied

The result from the quantum simulation is used for the application of the material properties of GB and while using the XFEM concept, the traction-separation law is used to calculate the appropriate maximum traction and separation value. The convergence study is conducted in terms of maximum stresses and found to be converging in the element size of $0.35 \mathrm{~nm}$. Tensile loading is applied with plane strain which has rate of $1 \mathrm{~nm} / \mathrm{s}$. In the figure below, constructed 3-dimensional structures of GB are shown along with anticipated crack propagation path.

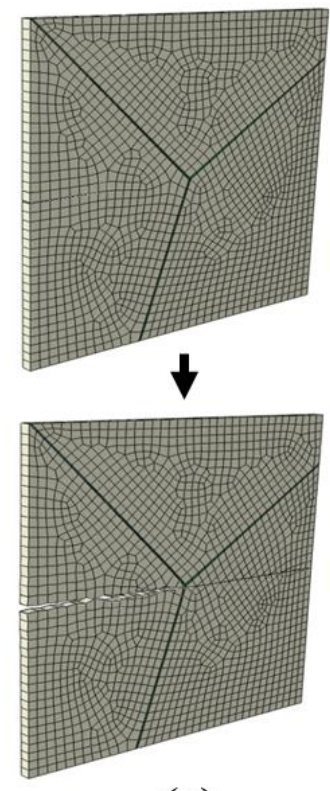

(a)

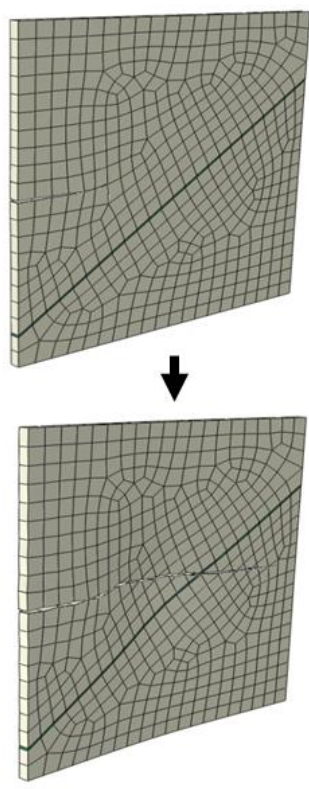

(b)

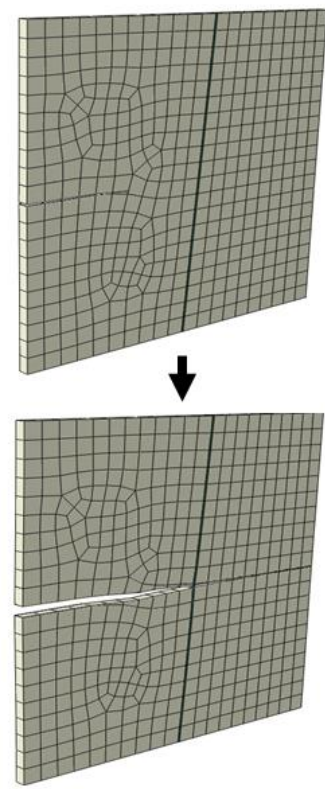

(c)

Figure 48: Three-dimensional models of GB region and the crack propagation samples

Relation for predicting peak tensile strength from $\mathrm{AB}$ initio CPMD framework is used to 
implement GB strength property in microscale simulation. The weak formulation of the boundary value problem in XFEM can be defined as

$$
\int_{\Omega} \boldsymbol{\sigma} \cdot \delta \mathbf{\varepsilon d} \Omega=\int_{\Omega} \mathbf{f}^{\mathrm{b}} \cdot \delta \mathbf{u d} \Omega+\int_{\Gamma_{\mathrm{t}}} \mathbf{f}^{\mathrm{t}} \cdot \delta \mathrm{ud} \Gamma
$$

where, $f^{b}$ is body force and $f^{t}$ is force from the external traction. Eq. (29) can re-written as $W^{\text {int }}=W^{\text {ext }}$.

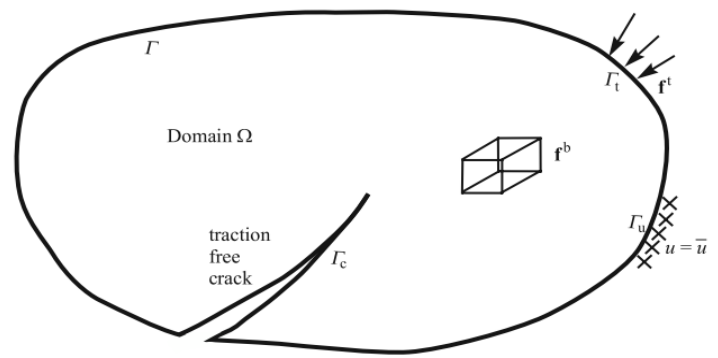

Figure 49: Illustration of boundary condition with crack

Maximum principal stress has been used for cohesive damage initiation criteria which is described in a function as

$$
f=\frac{\left\langle\sigma_{n}\right\rangle}{\sigma_{\max }^{0}}
$$

Initiation occurs when the maximum principal stress reaches a critical value at $f=1$. For the representation of crack-tip fields in three-dimensional computations, crack-tip enrichment functions are used in elements which contain the crack front. The crack front enrichment consists of functions which incorporate the radial and angular behavior of the two-dimensional asymptotic crack-tip displacement field ${ }^{18}$

$$
F_{j}(r, \theta)=\left[\sqrt{r} \sin \frac{\theta}{2}, \sqrt{r} \cos \frac{\theta}{2}, \sqrt{r} \sin \theta \sin \frac{\theta}{2}, \sqrt{r} \sin \theta \cos \frac{\theta}{2}\right]
$$

For the simulation of realistic fracture of $\mathrm{W}$ microstructure which involves multiple cracks in single microstructure as shown in figure below, the detail formulation of the study will be based on the ref. ${ }^{18,19}$.

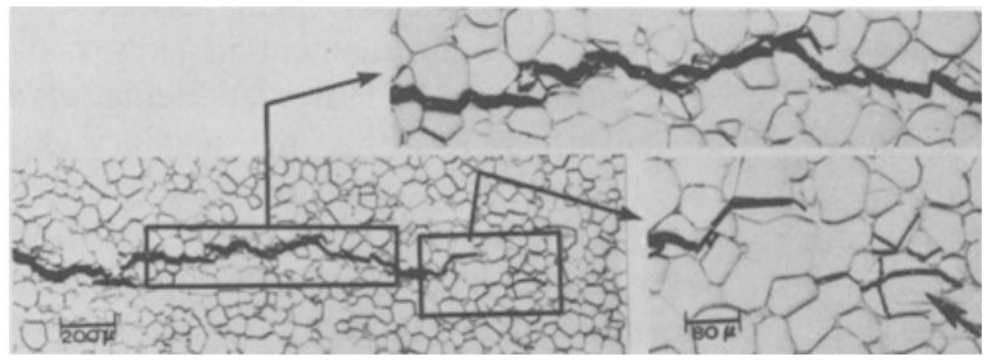

Figure 50: Fracture paths in W-Ni-Fe, (Churn and German, 1984) 
The XFEM simulation is conducted on one of the sample GB model and the crack propagation are observed successfully. Crack propagation of different cracks is tested. Standard initial crack, a void existing in arbitrary location within the microstructure, and both initial crack and a void are placed in the model, respectively, as shown in Figure 51 (a), (b), and (c).

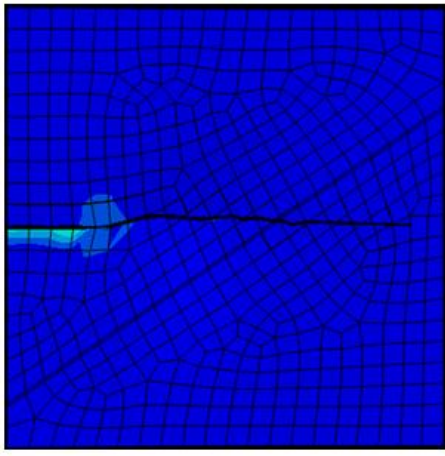

(a)

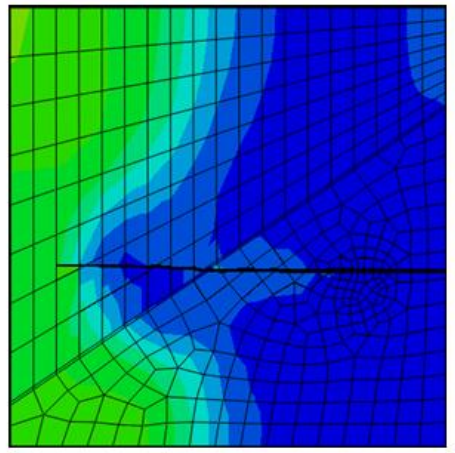

(b)

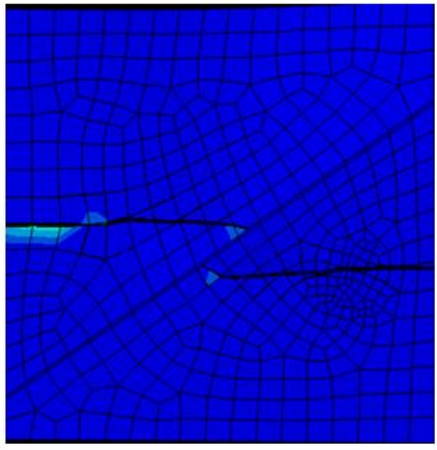

(c)

Figure 51: Crack propagation paths in microstructures with (a) single initial crack, (b) a void existing in arbitrary location, and (c) initial crack and a void existing in single model using XFEM technique

\section{$\underline{\text { Results and Discussion }}$}

Since polycrystalline tungsten's GBs have width that has order of tens of nanometer to hundreds of nanometer it is difficult to be examined by experimental study. By assuming three different fracture strength for GBs, which are (1) higher fracture strength than that of grains, (2) same fracture strength as grains, and (3) lower fracture strength than that of grains, qualitative result can be obtained with comparison of those three cases. With the result of these three assumptions of GBs, the true GBs strength comparing with grains can be predicted.

In this study, the cohesive finite element method (CFEM) has been employed to investigate two-dimensional fracture behavior of polycrystalline tungsten that has dimension of $400 \times 160$ micron. Three sets of simulation were performed with different GBs strength. Crack propagation directions and crack initiation time were obtained along with the energy dissipation and the crack length for analyses. With the relation between energy dissipation and crack length obtained from the result, an analytical relation has been proposed. Computational results were analyzed, and then compared with the proposed relation. Also, a surface energy relation developed in ref ${ }^{20}$ was used to find the sensitivity of fractions of primary crack and microcracks within the microstructure.

A specimen is taken from the microstructure extracted from an optical micrograph image of pure tungsten in ref ${ }^{21}$. then modeled with hyperelastic constitutive behavior based on experimental evidence, ${ }^{22,23}$, and ${ }^{24}$. Digitalizing process of microstructural morphology are described in the figure below. 
MORPHOLOGY 1

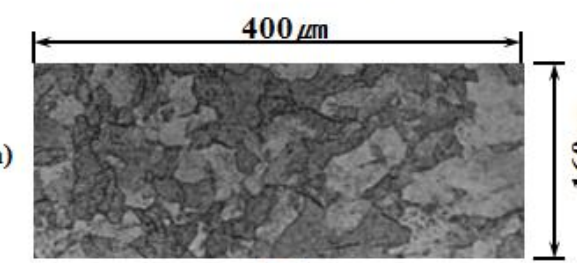

(b)

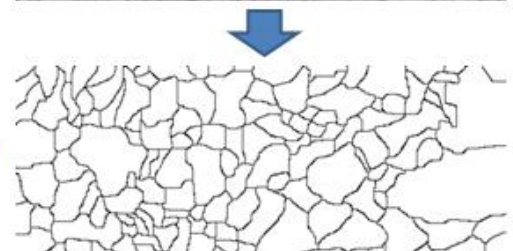

(c)

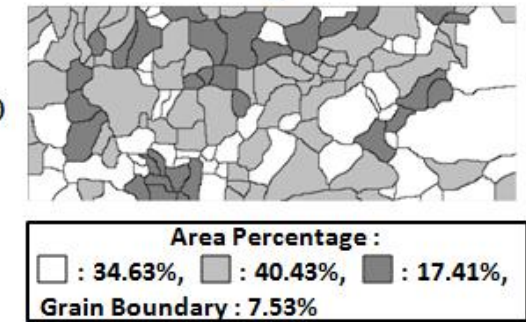

MORPHOLOGY 2
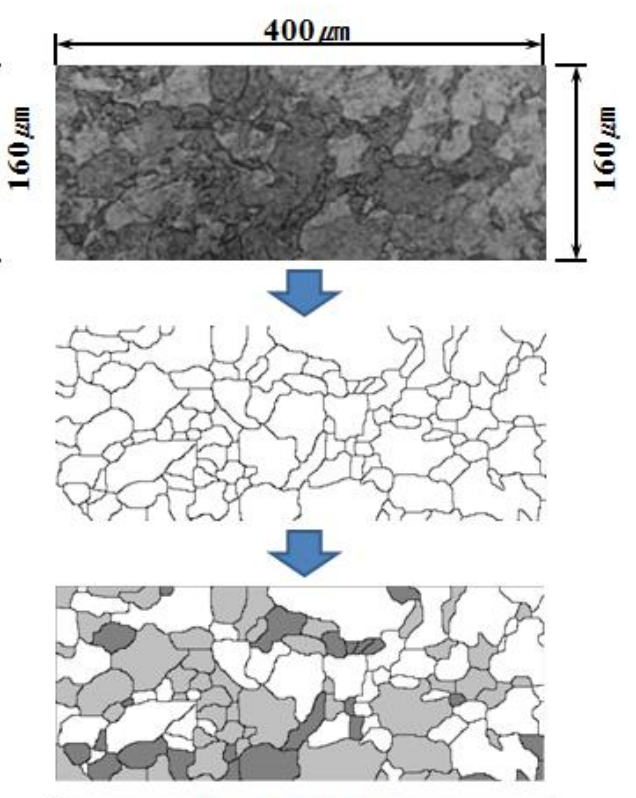

Area Percentage :

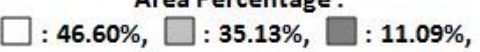

Grain Boundary: $7.18 \%$

Figure 52: Digitalization process of microstructural morphology with (a) original image of polycrystalline tungsten, (b) extraction of edges of GBs, and (c) completion of digitalization process by applying grain types into grains.

After obtaining the finite element meshes and determining the properties for the Tungsten microstructures, dynamic fracture analyses were performed at $1 \mathrm{~m} / \mathrm{s}$ loading rates. A Lagrangian finite deformation formulation is used to account for the finite strains and rotations in crack-tip regions. The principle of virtual work in stated as,

$$
\int_{V} s: \delta \mathrm{F} d v-\int_{S_{\mathrm{int}}} \mathrm{T} \cdot \delta \Delta d S=\int_{S_{\text {ext }}} \mathrm{T} \cdot \delta u d S-\int_{V} \rho \frac{\partial^{2} u}{\partial t^{2}} \cdot \delta u d V .
$$

Here $s: \delta \mathrm{F}=s^{i j} \delta \mathrm{F}_{j i}$ in Cartesian coordinates, $s$ is the first Piola-Kirchoff stress, $F$ is deformation gradient, $\Delta$ is the displacement jump across a pair of cohesive surfaces, $V$ is the volume, $S_{\text {ext }}$ and $S_{\text {int }}$ are external surface and internal cohesive surface area, respectively, and $\mathbf{T}$ is the traction applied on two coinciding material points at a position $\mathbf{x}$.

Generalized form of the cohesive laws (relation between $\mathbf{T}$ and $\boldsymbol{\Delta}$ ) with irreversibility can be considered as the one type of irreversible bilinear cohesive law. The surface energy dissipation per unit area $\Phi$ is function of separation vector $\Delta$ through a state variable defined as $\lambda=\sqrt{\left(\Delta_{n} / \Delta_{n c}\right)^{2}+\left(\Delta_{t} / \Delta_{t c}\right)^{2}}$ denoting that $\Delta_{n}=\mathbf{n} \cdot \boldsymbol{\Delta}$ and $\Delta_{t}=\mathbf{t} \cdot \boldsymbol{\Delta}$, respectively. $\Delta_{n c}$ is the critical normal separation at which the cohesive strength of an interface vanishes under conditions of pure normal deformation $\left(\Delta_{t}=0\right)$. Similarly, $\Delta_{t c}$ is the critical tangential separation at which the 
cohesive strength of an interface vanishes under conditions of pure shear deformation $\left(\Delta_{n}=0\right)$.

A parameter $\eta=\max \left\{\eta_{0}, \lambda_{u l}\right\}$ is defined and $\eta$ defines the stiffness of the original undamaged cohesive surface with $\eta_{0}$ which is the initial value and $\lambda_{u l}$ is the hitherto maximum value of $\lambda$. While $\eta_{0}$ is the characteristic value of effective separation $\lambda$ at which the effective traction $\sigma$ for a cohesive surface pair reaches the strength $\mathrm{T}_{\max }$ of the undamaged surface, $\lambda_{u l}$ is the critical level of $\lambda$ at which $\sigma$ reaches the reduced strength $T_{\max }(1-\eta) /\left(1-\eta_{0}\right)$ of the hitherto damaged cohesive surface pair. The specific form for $\Phi$ is taken as

$$
\Phi=\Phi(\lambda, \eta)= \begin{cases}\Phi_{0}\left(\frac{1-\eta}{1-\eta_{0}}\right)\left(\frac{\lambda^{2}}{\eta}\right), & \text { if } 0 \leq \lambda \leq \eta \\ \Phi_{0}\left(\frac{1-\eta}{1-\eta_{0}}\right)\left(1-\frac{(1-\lambda)^{2}}{1-\eta}\right), & \text { if } \eta<\lambda \leq 1 .\end{cases}
$$

This relation allows the traction to be defined as,

$$
\mathbf{T}=\frac{\partial \Phi}{\partial \boldsymbol{\Delta}}
$$

And the normal and shear traction components as,

$$
\mathrm{T}_{n}=\sigma(\lambda, \eta) \frac{\Delta_{n}}{\lambda \Delta_{n c}} \text { and } \mathrm{T}_{t}=\sigma(\lambda, \eta) \frac{\alpha \Delta_{t}}{\lambda \Delta_{t c}}
$$

In the above expressions, $\alpha=\Delta_{n c} / \Delta_{t c}$ and

$$
\sigma=\sqrt{\left(\mathrm{T}_{n}\right)^{2}+\left(\mathrm{T}_{t} / \alpha\right)^{2}}= \begin{cases}\left(\mathrm{T}_{\max } \frac{1-\eta}{1-\eta_{0}}\right) \frac{\lambda}{\eta}, & \text { if } 0 \leq \lambda \leq \eta ; \\ \left(\mathrm{T}_{\max } \frac{1-\eta}{1-\eta_{0}}\right) \frac{1-\lambda}{1-\eta}, & \text { if } \eta<\lambda \leq 1 ; . \\ 0, & \text { if } \lambda>1 .\end{cases}
$$

The work of separation for forming a fractured surface per unit surface area is

$$
\int_{0}^{\Delta_{c}} \mathbf{T} \cdot \mathrm{d} \Delta=\frac{1}{2} \mathrm{~T}_{n}^{\max } \Delta_{n c}=\frac{1}{2} \alpha \mathrm{T}_{n}^{\max } \Delta_{t c}=\Phi\left(1, \eta_{0}\right)=\Phi_{0}
$$

While the bilinear relationship between $\sigma$ and $\lambda$ embodied in the above formulation is illustrated in the Figure, the variation of $\Phi$ is also shown. Based on Eq. (39) the amount of work that has been dissipated, $\Phi_{d}$, is 


$$
\Phi_{d}(\lambda, \eta)=\left\{\begin{array}{l}
0, \text { if } \lambda \leq \eta_{0} \\
\Phi\left(\eta, \eta_{0}\right)-\Phi(\eta, \eta)=\frac{\eta-\eta_{0}}{1-\eta_{0}} \Phi_{0}, \text { if } \eta_{0}<\lambda \leq \eta \\
\Phi\left(\lambda, \eta_{0}\right)-\Phi(\lambda, \eta)=\frac{\lambda-\eta_{0}}{1-\eta_{0}} \Phi_{0}, \text { if } \eta<\lambda \leq 1 ; \\
\Phi_{0}, \text { if } \lambda>1 ;
\end{array}\right.
$$

When full separation is achieved, $\Phi_{d}(1, \eta)=\Phi_{0}$. The overall cohesive energy dissipated $\phi_{d}$ is the sum of energy dissipated in all the damaged cohesive surfaces, i.e. $\phi_{d}=\int_{S_{d}} \Phi_{d} \mathrm{~d} S$, where $S_{d}$ represents total surface area of cohesive surfaces with separation. $\Phi_{d}$ is partly converted into the surface energy and partly spent on causing damage in the material adjacent to crack surfaces through microcrack formation that is not based on a pre-specified criterion. A unique damage parameter can be defined to phenomenologically track the progressive softening of cohesive surfaces interspersed throughout the composite microstructure. This parameter D is defined such that

$$
\mathrm{D}=\frac{\Phi_{d}}{\Phi_{0}}
$$

Note that $0 \leq \mathrm{D} \leq 1$, with $\mathrm{D}=0$ indicating fully recoverable interfacial separation and $\mathrm{D}=1$ signifying complete separation or total fracture. The spatial and time variation of $\mathrm{D}=\mathrm{D}(\mathbf{x}, t)$ allows the distribution and evolution of damage in various microstructures to be analyzed.

The tungsten microstructures include three grain types and one grain boundary. From the one type of grain's elastic modulus, other grain has 5\% less and another grain has 5\% greater in elastic modulus of the first grain type. For the GB there are 3 assumptions. First case has elastic modulus of the GB to be $10 \%$ less than the average value (average of grains). Another case has elastic modulus of the GB to be same as the average value. Lastly, the third case has elastic modulus of the GB to be $10 \%$ greater than the average value. Since the fracture strength increases as the elastic modulus decreases, fracture strength of all grains and GBs are correspondingly adjusted according to the above percentages. The area that covers the microstructures (effective zone) has the homogenized property of all phases. The homogenized property is calculated based on volume fraction of each phase and their properties.

Cohesive energy dissipation plots for three cases (high fracture strength GB, medium fracture strength GB, and low fracture strength GB) are shown in the figure below. The figures clearly show different shape of energy release points for different cases at time $t=155$ nanoseconds. In the initiation of crack propagation, very small portion of crack lines are detected for all three cases with similar crack pattern. The primary crack length at $\mathrm{t}=125 \mathrm{nsec}$ is approximately $12 \sim 15 \mu \mathrm{m}$ for all three cases. We can conclude that the time of crack initiation is not significantly dependent on GB's properties. However, pattern of energy release after crack initiation show distinguishing features for different cases. 

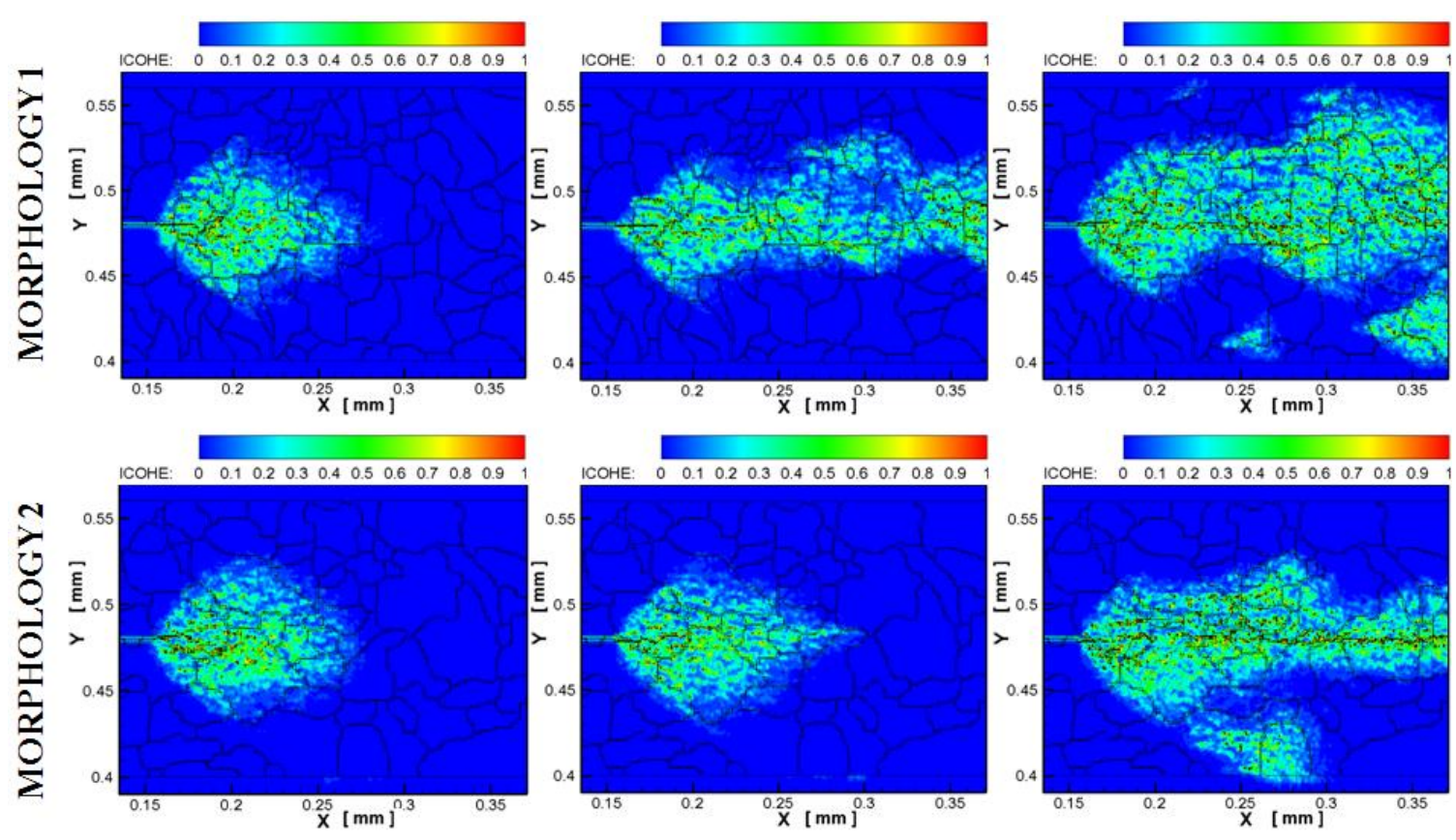

(a)

(b)

(c)

Figure 53: Cohesive energy dissipation plots for the three cases of simulation which have (a) high fracture strength GBs, (b) medium fracture strength GBs, and (c) low fracture strength GBs at $\mathrm{t}=155$ nanosecond (ICOHE is the cohesive energy dissipation that was scaled down to have range of 0 to 1 )

Damage plots shown in the figure 45 indicate both primary crack and microcracks with red lines representing approximated primary crack propagation path along the microstructures. The crack propagations are combined with inter-granular failure and trans-granular failure for all three cases of different GBs' strength. While GBs' strength does not affect crack initiation time significantly, it has significant effect on the primary crack propagation and microcracking in terms of crack length. 


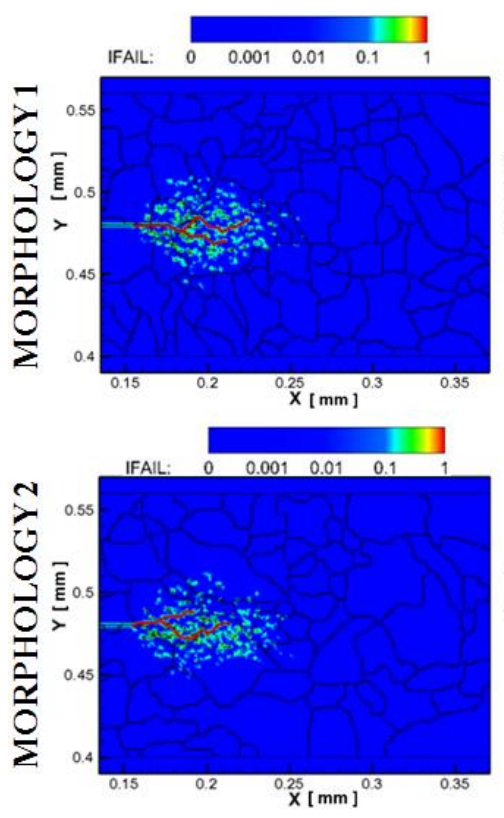

(a)
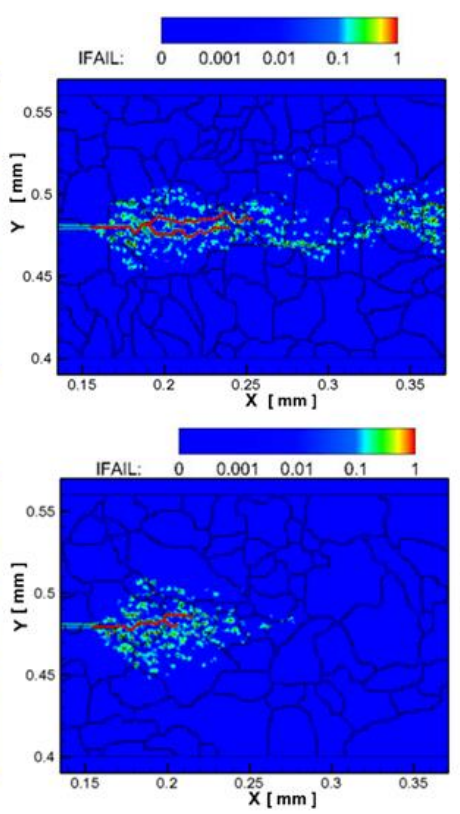

(b)

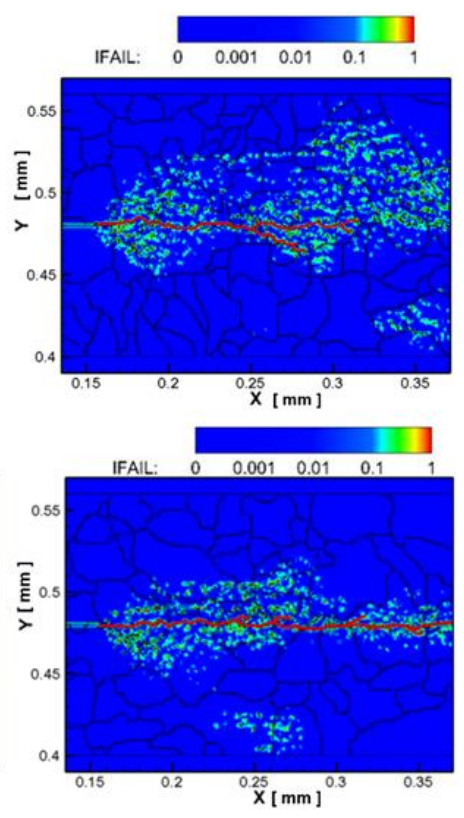

(c)

Figure 54: Damage plots of three different cases of simulation which include (a) high fracture strength GBs, (b) medium fracture strength GBs, and (c) low fracture strength GBs at $\mathrm{t}=155$ nanosecond (IFAIL is the Damage variable with range of 0 to 1 )

This phenomenon can also be observed in the next figure. The fracture behavior in these cases involves crack branching as well as crack propagation. Branching of crack occurs in different regions of the microstructure in three cases. As strength of GB becomes lower, the region of branching moves further away from the crack tip. In other words, the fraction of microcracking in total crack density becomes larger as the strength of GB becomes lower.

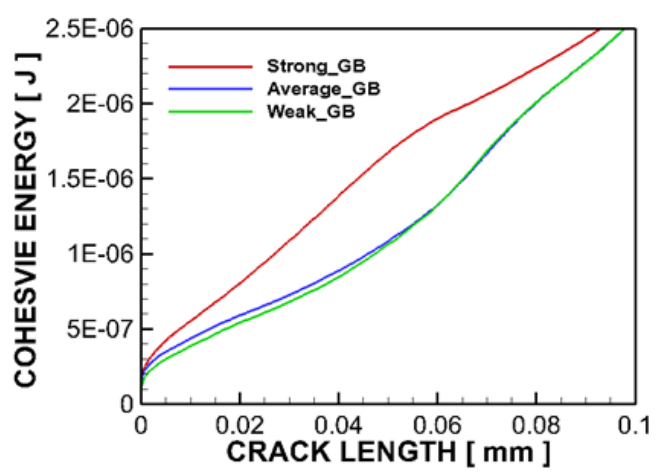

(a)

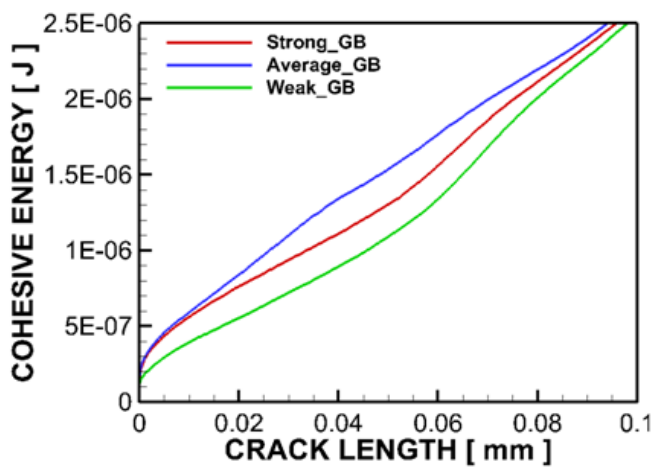

(b)

Figure 55: Plot of cohesive energy as a function of crack length for (a) morphology 1 and (b) morphology 2

Development of crack length from grains and interface as a function of time is shown in the 
figure 54(a). Obviously, crack length in interface region becomes greater when GB has lower strength. However, the figure indicates that low strength of GB is also affecting the crack development in grain region, even though properties of grains are identical for all three cases.

Cohesive energy dissipation as functions of time is shown in figure 55(b). We can observe that cohesive energy dissipation with respect to time is very similar to each other for different strength GB cases.
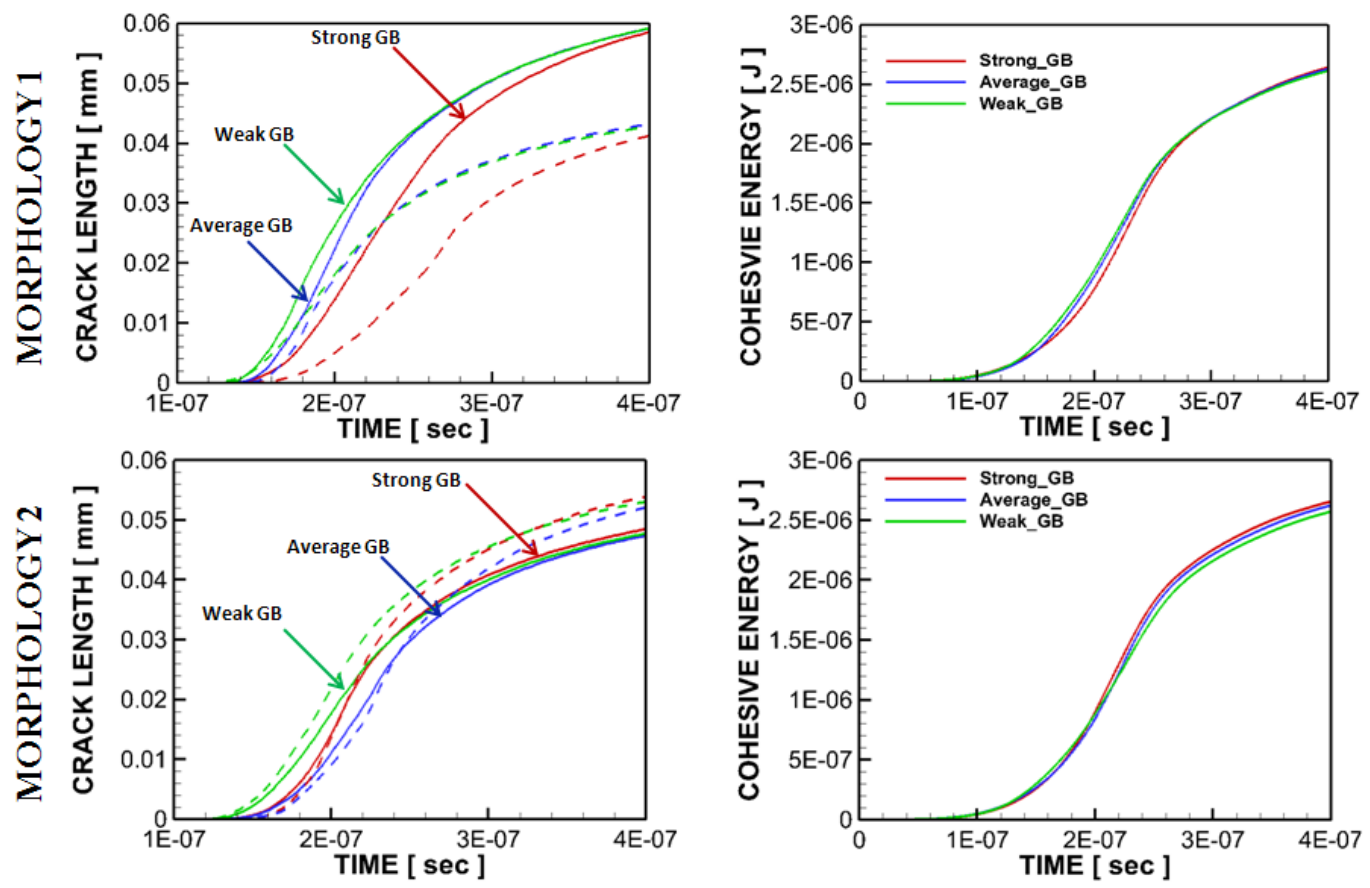

(a)

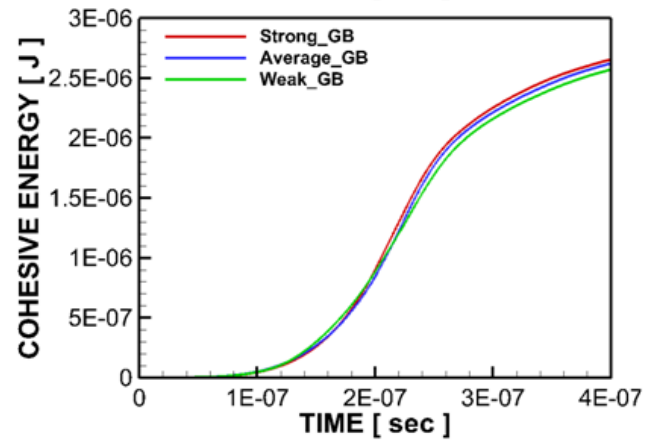

(b)

Figure 56: (a) Plot of crack length as a function of time for grains and interface where solid line indicates grains and dotted line indicates interface, (b) plot of cohesive energy dissipation as a function of time 

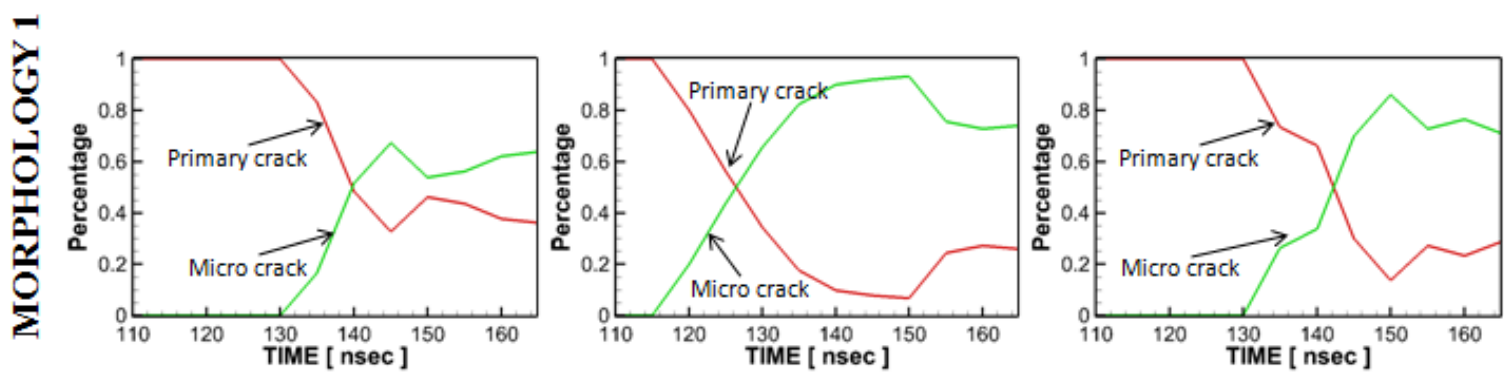

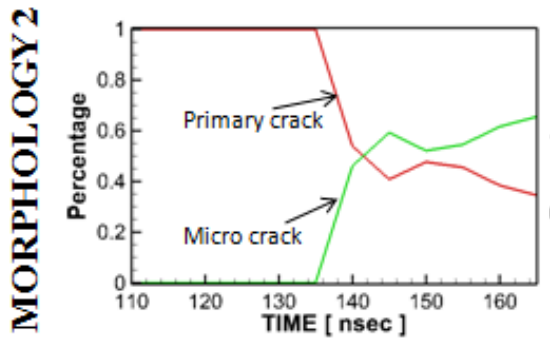

(a)

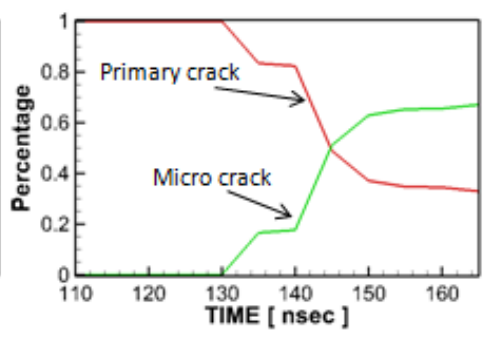

(b)

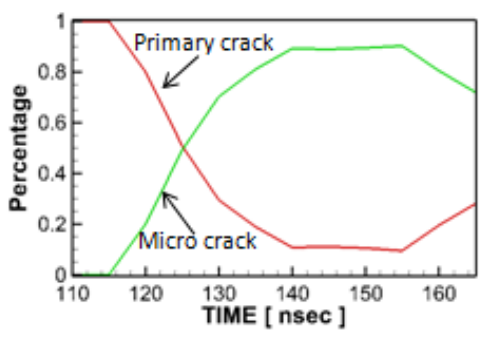

(c)

Figure 57: Percentages of primary crack and microcracks for different cases of simulation which contain (a) high fracture strength GBs, (b) medium fracture strength GBs, and (c) low fracture strength GBs.

The failure of microstructure under the loading occurs in two major forms; primary crack and microcracks. By categorizing these two forms of cracks, the pattern of the fracture behavior can be analyzed. It is convenient to analyze and find the sensitivity of primary crack's fraction within the microstructures using the pre-established relation using surface energy. Rice and Freiman have established a relation that will separate two types of cracks $^{20}$.

$$
\gamma=\gamma_{p c}\left[1-\left(G / G_{s}\right)\right]^{m}+\gamma_{\mu}
$$

In this equation, $\gamma_{p c}$ and $\gamma_{\mu}$ represent polycrystalline surface energy and microcrack formation energy, respectively and $m$ is the coefficient that separates two types of cracks. The term, $\gamma_{p c}\left[1-\left(G / G_{s}\right)\right]^{m}$ is used to denote contribution of a polycrystalline microstructure's grain size dependent on primary crack propagation fracture energy with $G$ being the grain size where crack initiates, $G_{\mathrm{s}}$ being the average microstructural grain size, and $\mathrm{m}$ being an exponent parameter.

After obtaining the damage plots of several time periods the percentages of primary crack and microcracks for each time periods were plotted to calculate the sensitivity value, $\mathrm{m}$. The figure above indicates that the initiating crack comes from primary crack and microcracks begins to overwhelm the primary crack at time, $\mathrm{t}=125 \sim 145 \mathrm{nsec}$. These obtained percentages are brought into the surface energy relation to find the value of $\mathrm{m}$, which is shown in the next figure. While the calculated $\mathrm{m}$ values are plotted with symbols the average sensitivity value for pure tungsten is drawn as a solid line, which have $\mathrm{m}=14.06$ for morphology 1 and $\mathrm{m}=13.48$ for morphology 2 . 


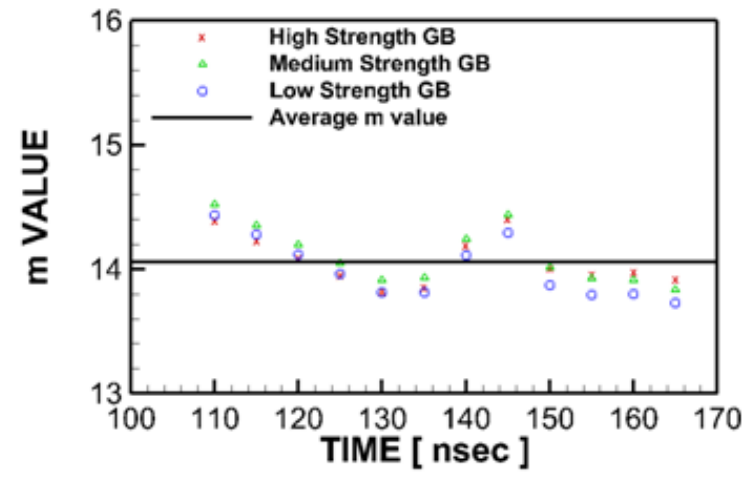

(a)

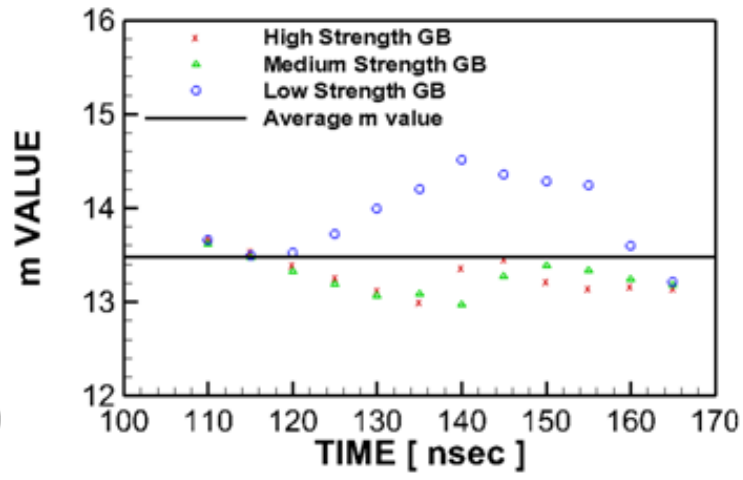

(b)

Figure 58: Evaluated $M$ values with distributed $m$ values of three cases of study (a) on morphology 1, and (b) morphology 2

From the definition of traction-separation law, the separation parameter can be described as

$$
\Delta=\frac{2 \cdot \Phi_{0}}{\mathrm{~T}_{\max }} .
$$

A normalized length scale of the models can be calculated from the area of microstructure divided by area of four triangular elements, which is the same as one quadrilateral element as

$$
L=\frac{a \cdot b}{h^{2}} \text {. }
$$

In the above equation, $h$ is the characteristic element size. By multiplication of separation parameter and normalized length scale with the cohesive energy per unit area, the energy dissipation per unit crack length can be obtained. Therefore, the relation between the energy dissipation and crack density is

$$
C E=25 \cdot \frac{\Phi_{0}^{2} \cdot(a \cdot b)}{\mathrm{T}_{\text {max }} \cdot h^{2}} \cdot C D
$$

where CE indicates energy dissipation and CD indicates crack density.

The proposed relation between energy dissipation and crack density is compared with simulation results as shown in the figure below. We can observe that case of low strength GB simulation is mostly coincident with the developed relation. This relation can also be used to compare the GB properties. 


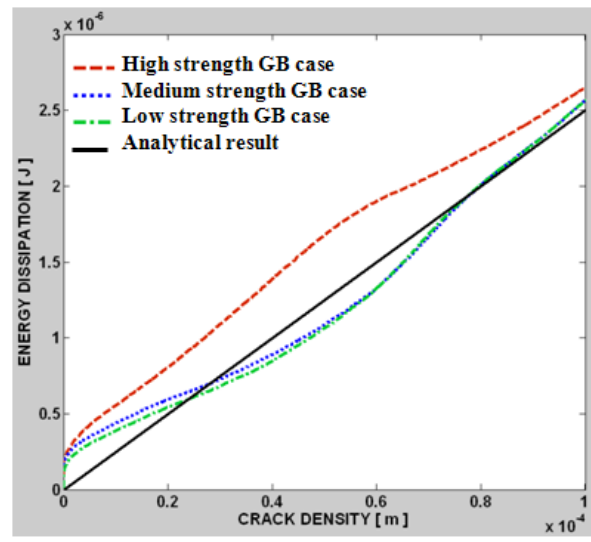

(a)

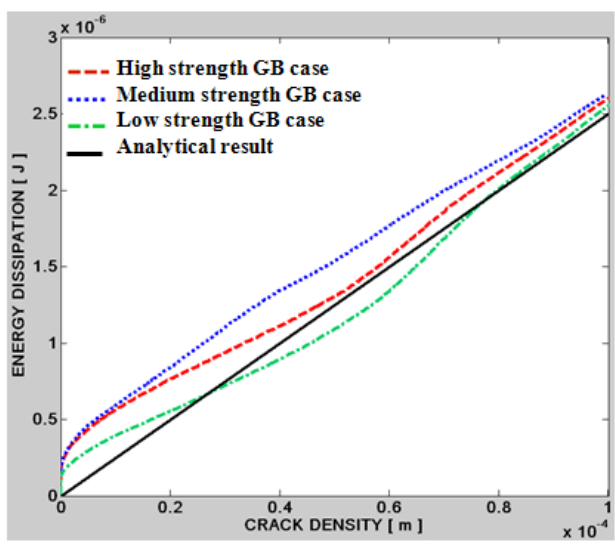

(b)

Figure 59: Comparison of the analytical solution with simulation results for (a) morphology 1 and (b) morphology 2

\section{§6. Simulation of Multiscale Model of Grain Boundary in the Fracture Using Cohesive Finite Element Method}

Strength properties of grain boundaries in angstrom-unit level have been investigated using the first-principle method to find a relation for predicting the maximum tensile stress of interface. While quantum mechanical calculation has been used to derive the strength properties of interface, the cohesive finite element method is considered to investigate actual interfacial fracture in microscale. In past decades, several researches were conducted to accurately predict crack propagation. Failure criterions were proposed using the stress, strain energy, and energy release rate $^{25-27}$. Also, analytical solutions for crack propagation are developed to mathematically represent the fracture behavior. With existing analytical solutions and failure criterions, however, it is still difficult to accurately predict the crack propagation in polycrystalline material. One of the reasons is the uncertainty of grain boundary strength property. Due to small scale of the grain boundary in polycrystalline material, it is difficult to obtain strength property of grain boundary. Also, modeling of multiscale model of interface for fracture is not easy task. Thus, it is necessary to investigate interfacial fracture in different scales based on the obtained interfacial strength property. In order to take account of thickness of interface, grain boundaries are modeled in three different widths. Interfacial models for simulation are built in micrometers and millimeters to find any scale effect in the fracture. Overall simulation setups and boundary conditions are illustrated in the figure below. 


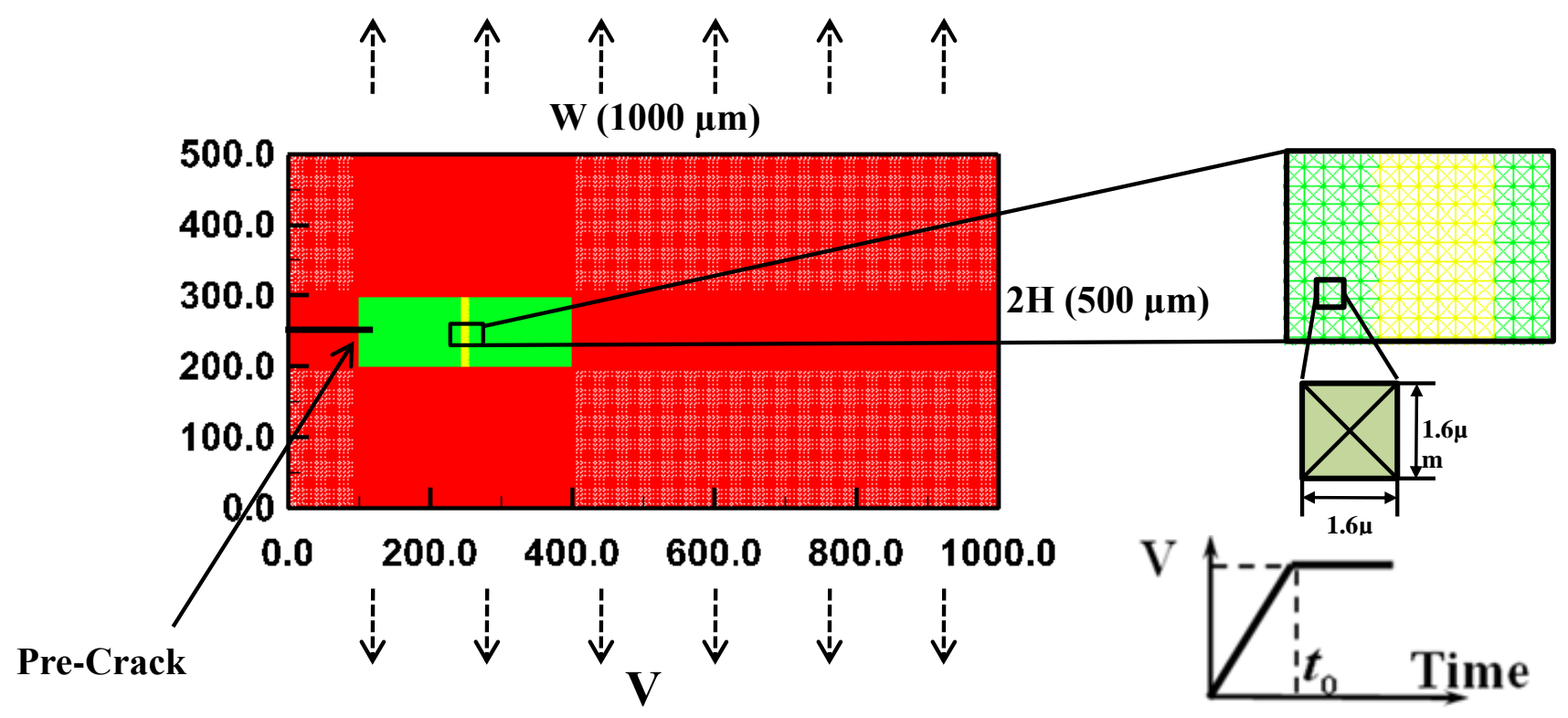

Figure 60: Schematic illustration simulation setup and boundary condition for the cohesive finite element method

From the fracture of tungsten interface, (N. Muskhelishvili, 1966) have developed a model to describe relation between applied stress and the plastic zone stress. The ratio between applying stress and plastic zone stress is defined as,

$$
\begin{aligned}
& \frac{\sigma}{\sigma_{1}}=\frac{(\rho / a)^{1 / 2}}{2+(\rho / a)^{1 / 2}}+\frac{4}{\pi\left(2+(\rho / a)^{1 / 2}\right)} \\
& \times \arccos \left[\frac{1-\rho / a}{1+s / a-(\rho / a)^{1 / 2}\left\{\left[(1+s / a)^{2}-1+\rho / a\right]^{1 / 2}-\chi(\rho / a) s / a\left(1+(s / a)^{1 / 2}\right)\right\}}\right]
\end{aligned}
$$

Initial crack length and height are denoted as $a$ and $b$, respectively, and $s$ is plastic zone length. The crack-root radius is defined as

$$
\rho=\frac{b^{2}}{a}
$$

For the boundary condition of plane strain, yielding from an embedded crack with finite root-radius and loaded in uniform tension is used. The relation between applied stress and the plastic zone stress, shown above, is constructed to derive approximate analytical formula for the dependence of the plastic zone size and the crack opening displacement on the ratio of the applied and friction stress. Crack dimension and the plastic zone length are schematically illustrated in 
figure below.

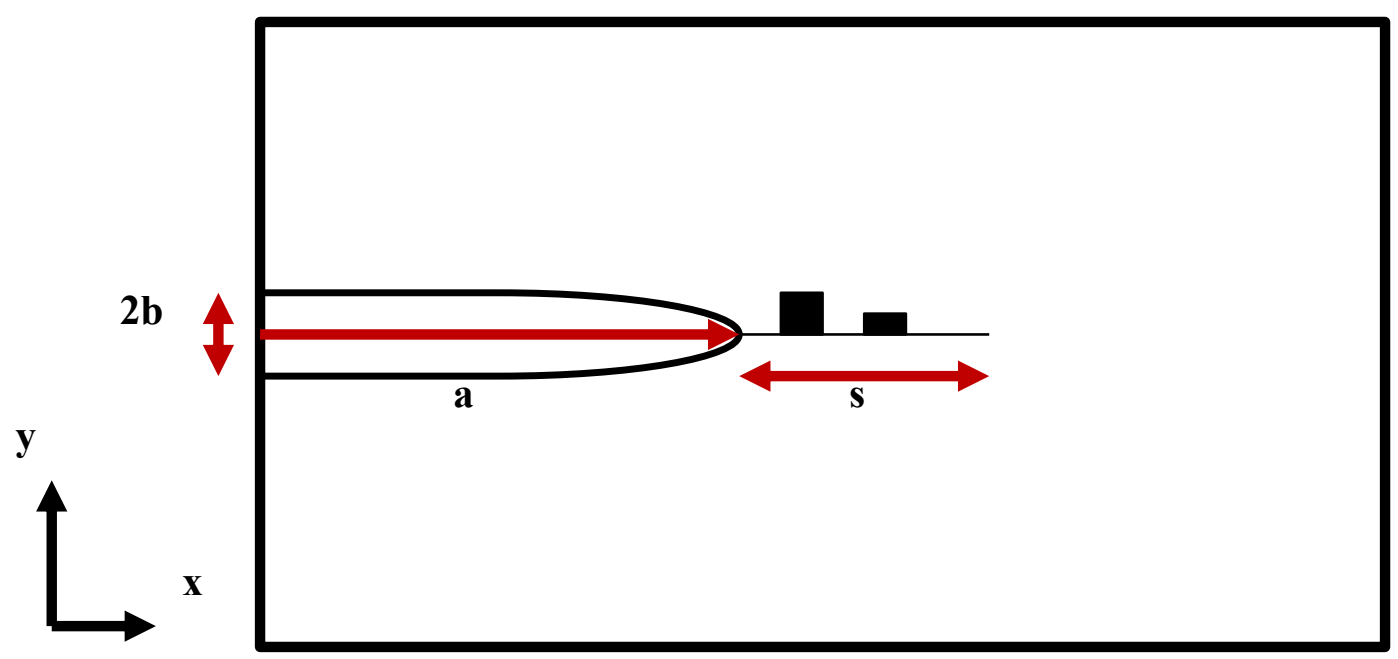

Figure 61: Schematic illustration of the embedded crack with plastic zone

In performing simulations using cohesive finite element method, it is difficult to capture the data regarding plastic zone length directly. In (F. Caputo and et al., 2013), an analytical relationship, which links, in a closed form, plastic zone size to the parameters and which is able to determine the plastic zone size at the crack tip of both physically short cracks and long cracks has been proposed as

$$
r_{p}=\left(\frac{K \sqrt{2 a}(1-2 v)}{m \cdot\left(\sqrt{4 a \pi \cdot s_{y}^{2}-3 B^{2} K^{2}}-B K(1-2 v)\right)}\right)^{\frac{1}{p}}
$$

Here, $r_{p}$ is plastic zone length and the yield strength is denoted as $s_{y} . K$ is stress intensity factor. $\mathrm{v}$ is Poisson's ratio and $\mathrm{p}, \mathrm{m}, \mathrm{B}$ are stress parameters in this formulation. It is important to note that the model does not necessarily have to be plastic model to have the plastic zone. In the work of (V. Vitek, 1975), isotropic elastic model is used to derive the plastic zone length.

In the conducting of simulations using cohesive finite element method, choice of mesh or element size is critical for obtaining accurate result. Convergence test has been performed to find appropriate element size based on the convergence criteria developed in ${ }^{28}$. The overall concept of the convergence criteria is to be between two bounds where first bound is that the element size should be much smaller than the cohesive zone size and the second bound that the macroscopic stiffness reduction due to cohesive separation along element boundaries if the initial stiffness of cohesive surface is finite. Based upon the satisfied range by this convergence criteria element sizes are varied to find the accurate and efficient mesh size. Figure below is a plot for showing the result as a function of element size and maximum element size. It is clear that the element size of $0.5 \sim 1$ $\mu \mathrm{m}$ is showing gradual increase of damage while bigger element size is showing steep incremental result. 


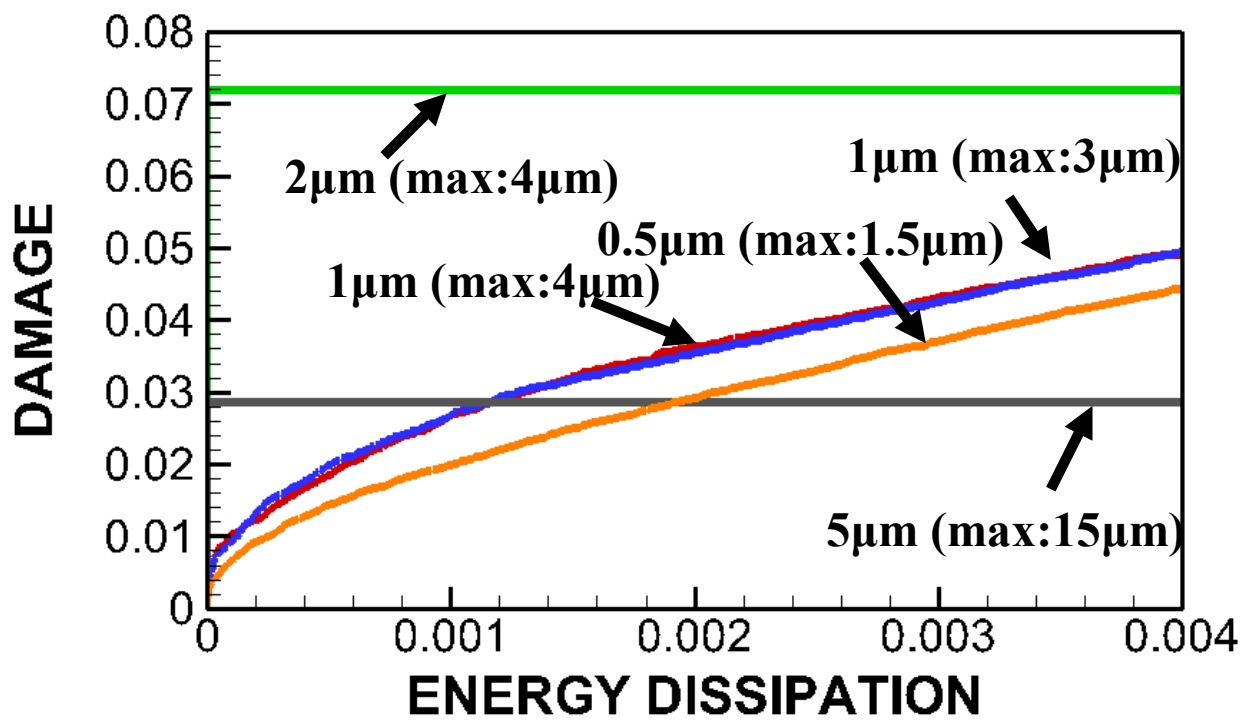

Figure 62: Damage as a function of energy dissipation for different element size and maximum element size in convergence study 


\section{Conclusions}

The key achievements and conclusions are listed below:

a. A new high-temperature grain boundary segregation theory and a new model (formulation) have been developed for W-based ternary alloys. An analytical method was also developed in addition to the numerical approach. The numerical and analytical methods have been tested and refined.

b. We have conducted a systematic numerical experiment for W-Ni-X alloys (where $\mathrm{X}$ is a fictive element) to identify the key thermodynamic factors that control grain boundary segregation behaviors in W-based ternary alloys.

c. We have modeled W-Ni-Fe, W-Ni-Ti, W-Ni-Co, W-Ni-Cr, W-Ni-Zr and W-Ni-Nb ternary alloys with real CALPHAD and thermodynamic data.

d. We have conducted controlled sintering experiments validate our thermodynamic theory and modeling results using a series of ternary $\mathrm{W}-0.5 \mathrm{Ni}-0.5 \mathrm{X}$ at. $\%$ (where $\mathrm{X}=\mathrm{Cr}, \mathrm{Zr}, \mathrm{Co}, \mathrm{Fe}, \mathrm{Nb}$, Ti) alloys, along with binary $\mathrm{W}-0.5 \mathrm{Ni}$ and $\mathrm{W}-1 \mathrm{Ni}$ alloys as references, as our model experimental systems.

e. We have investigated the interfacial property of Ni-doped $\mathrm{W}$ using quantum simulation.

f. We have further investigated the Role of grain boundary in the fracture using 3-D extended finite element method.

In summary, we have developed thermodynamic theories and models to predict high-temperature GB segregation in $\mathrm{W}$ alloys, conducted multiscale modeling (at continuum, atomistic and quantum-mechanical levels) to link GB segregation with GB embrittlement, and validated the developed theories and modeling results via selected experiments. The most significant contribution of this project is the successful development of a theoretical framework in combination with a multiscale modeling strategy for predicting GB segregation and embrittlement in $\mathrm{W}$ based alloys. 


\section{Graphic Materials List}

Figure 1: Computed isothermal sections of a computed "GB diagram" for $(\mathrm{Ni}+\mathrm{Cr})$ co-doped $\mathrm{W}$ at (left) $1400 \mathrm{~K}$ and (right) $1500 \mathrm{~K}$, respectively. Contour plots of constant $\lambda$ values are shown. The computed $\lambda$ value corresponds to the degree of disorder at GBs, which is coupled with the adsorption level.

Figure 2: A computed isothermal section of a GB "phase" diagram for $(\mathrm{Ni}+\mathrm{Cr})$ co-doped $\mathrm{W}$ at $1600 \mathrm{~K}$. The contour plots of constant $\lambda$ values are shown. The computed solidus line is also shown.

Figure 3: Comparison of the estimated $\lambda$ values for Ni-doped Mo at $1400 \mathrm{~K}$ using different (approximated) analytical solutions according to: (a) Eq. (11) ref [4]; (b) Eq. (10) as a first-order approximation; and (c) a second-order approximation of Eq. (8) using the Taylor expansion.

Figure 4: A computed $\lambda$ diagram using an approximate analytical solution [Eq. (10)] for $(\mathrm{Ni}+\mathrm{Cr})$ co-doped $\mathrm{W}$ at $1400 \mathrm{~K}$. The results are generally consistent the "exact" solution computed by numerical methods shown in Fig. 2.

Figure 5: (a) The $\mathrm{X}_{\text {film }}$ map of $\mathrm{W}-\mathrm{Ni}-\mathrm{Cr}$ system.

Figure 6: The physical properties of transition metals.

Figure 7: Computed $\lambda$ diagrams for the W-Ni-X system assuming: $\Omega_{W-X}^{\text {liquid }}=\Omega_{W-X}^{b c c}=\Omega_{N i-X}^{\text {liquid }}=$ $\Omega_{N i-X}^{b c c}=0$, and $\mathrm{T}=1673 \mathrm{~K}$. The values of $T_{\mathrm{m}}{ }^{\mathrm{X}}$ are labeled. Red lines are the solidus lines.

Figure 8: Computed $X_{\text {film }}$ maps for the W-Ni-X system assuming: $\Omega_{W-X}^{\text {liquid }}=\Omega_{W-X}^{b c c}=\Omega_{N i-X}^{\text {liquid }}=$ $\Omega_{N i-X}^{b c c}=0$, and $\mathrm{T}=1673 \mathrm{~K}$.

Figure 9: Computed GB $(\lambda)$ diagrams for the W-Ni-X system for: $\Omega_{W-X}^{\text {liquid }}=\Omega_{N i-X}^{\text {liquid }}=\Omega_{N i-X}^{b c c}=0, \mathrm{~T}=$ $1673 \mathrm{~K}$ and $T_{\mathrm{m}}{ }^{\mathrm{X}}=2000 \mathrm{~K}$. The values of $\Omega_{W-X}^{b c c}$ are labeled.

Figure 10: Computed $X_{\text {film }}$ maps for the W-Ni-X system assuming: $\Omega_{W-X}^{\text {liquid }}=\Omega_{N i-X}^{\text {liquid }}=\Omega_{N i-X}^{b c c}=0, \mathrm{~T}$ $=1673 \mathrm{~K}$ and $T_{\mathrm{m}}{ }^{\mathrm{X}}=2000 \mathrm{~K}$. The values of $\Omega_{W-X}^{b c c}$ are labeled.

Figure 11: Computed GB $\lambda$ diagrams for the W-Ni-X system for: $\Omega_{W-X}^{b c c}=\Omega_{N i-X}^{\text {liquid }}=\Omega_{N i-X}^{b c c}=0, \mathrm{~T}=$ $1673 \mathrm{~K}$, and $T_{\mathrm{m}}^{\mathrm{X}}=2000 \mathrm{~K}$. The values of $\Omega_{W-X}^{\text {liquid }}$ are labeled.

Figure 12: Computed $X_{\text {film }}$ maps for the W-Ni-X system assuming: $\Omega_{W-X}^{b c c}=\Omega_{N i-X}^{\text {liquid }},=\Omega_{N i-X}^{b c c}=0$, $\mathrm{T}=1673 \mathrm{~K}$, and $T_{\mathrm{m}}{ }^{\mathrm{X}}=2000 \mathrm{~K}$. The values of $\Omega_{W-X}^{\text {liquid }}$ are labeled.

Figure 13: Computed GB $(\lambda)$ diagrams for the W-Ni-X system for: $\Omega_{N i-X}^{\text {liquid }}=\Omega_{N i-X}^{b c c}=0$, $\Omega_{W-X}^{b c c}-\Omega_{W-X}^{\text {liquid }}=50 \mathrm{KJ} / \mathrm{mol}, \mathrm{T}=1673 \mathrm{~K}$ and $T_{\mathrm{m}}{ }^{\mathrm{X}}=2000 \mathrm{~K}$. The values of $\Omega_{W-X}^{\text {liquid }}$ and 
$\Omega_{W-X}^{b c c}$ are labeled.

Figure 14: Computed $X_{\text {film }}$ maps for the W-Ni-X system assuming: $\Omega_{N i-X}^{\text {liquid }}=\Omega_{N i-X}^{b c c}=0$, $\Omega_{W-X}^{b c c}-\Omega_{W-X}^{\text {liquid }}=50 \mathrm{KJ} / \mathrm{mol}, \mathrm{T}=1673 \mathrm{~K}$ and $T_{\mathrm{m}}{ }^{\mathrm{X}}=2000 \mathrm{~K}$. The values of $\Omega_{W-X}^{\text {liquid }}$ and $\Omega_{W-X}^{b c c}$ are labeled.

Figure 15: a) Computed $\lambda$ diagram of $\mathrm{W}-\mathrm{Ni}-\mathrm{Cr}$ system b) $X_{\text {film }}$ maps.

Figure 16: a) Computed $\lambda$ diagrams of $\mathrm{W}-\mathrm{Ni}-\mathrm{Cr}$ system b) $X_{\text {film }}$ maps.

Figure 17: Computed $\lambda$ diagrams of $\mathrm{W}-\mathrm{Ni}-\mathrm{X}$ system at $1400{ }^{\circ} \mathrm{C}$ and $1600{ }^{\circ} \mathrm{C}$.

Figure 18: Computed $\lambda$ diagrams considering multi-component system of $\mathrm{W}-\mathrm{Ni}-\mathrm{X}$ system at 1400 ${ }^{\circ} \mathrm{C}$ and $1600{ }^{\circ} \mathrm{C}$.

Figure 19: Experimental Data: (a) average density increase of $\mathrm{W}-0.5 \mathrm{Ni}-0.5 \mathrm{X}$ at. $\%(\mathrm{X}=\mathrm{Cr}, \mathrm{Zr}$, $\mathrm{Co}, \mathrm{Fe}, \mathrm{Nb}, \mathrm{Ti})$ specimens, sintered at $1473 \mathrm{~K}$ for 5 hours and (b) average density increase of $\mathrm{W}-0.5 \mathrm{Ni}-0.5 \mathrm{X}$ at. \% (X $=\mathrm{Cr}, \mathrm{Zr}, \mathrm{Co}, \mathrm{Fe}, \mathrm{Nb}, \mathrm{Ti})$ specimens sintered at $1573 \mathrm{~K}$ for 2 hours.

Figure 20: A Critical Model-Experimental Comparison: Computed $\lambda$ values (extracted from the GB diagrams presented in \$2.2) vs. the experimentally-observed sintering rates (density increases after sintering at $1573 \mathrm{~K}$ for 2 hours) of ternary $\mathrm{W}-0.5 \mathrm{Ni}-0.5 \mathrm{X}$ at. $\%(\mathrm{X}=\mathrm{Cr}$, $\mathrm{Zr}, \mathrm{Co}, \mathrm{Fe}, \mathrm{Nb}, \mathrm{Ti}$ ), along with binary $\mathrm{W}-0.5 \mathrm{Ni}$ at. $\%, \mathrm{~W}-1 \% \mathrm{Ni}$ alloys as reference. The experimentally-observed sintering rate is an indication of the level of GB disorder. Thus, the correlation between the computed $\lambda$ values and the experimentally-observed sintering rates help validate our thermodynamic theories/modeling.

Figure 21: Convergence study on the number of k-point for integration over Brillouin zone

Figure 22: Distribution of bond lengths for $\mathrm{W}-\mathrm{W}$ and $\mathrm{W}-\mathrm{Ni}$ over different number of k-point for

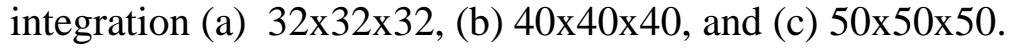

Figure 23: (a) High resolution transmission electron microscopy image of nickel-doped tungsten and (b) schematic illustration of crystal structure which represents two differently oriented tungsten phases with nickel added grain boundary.

Figure 24: Illustration of atomic supercells analyzed in the present work with (a)two differently oriented W phases, (b) two differently oriented W phases with Ni added GB thickness of $0.3 \mathrm{~nm}$, (c) illustration of different atomic fractions of $\mathrm{Ni}$ in W GB, (d) illustration of atomic supercells with two differently oriented $\mathrm{W}$ phases with Ni added GB thickness of $0.6 \mathrm{~nm}$, (e) illustration of different atomic fraction of $\mathrm{Ni}$ in $\mathrm{W}$ GB.

Figure 25: Stress-strain plot of pure W (GB thickness=0nm).

Figure 26: (a) Stress-strain relation of unsaturated Ni-doped W (GB thickness $=0.3 \mathrm{~nm})$, (b) comparison of yield stress and peaks of the curves.

Figure 27: (a) Stress-strain relation of saturated Ni-doped W (GB thickness=0.6nm), (b) comparison of yield stress and peaks of the curves. 
Figure 28: Density of States plots of (a) pure W, (b) unsaturated Ni-doped W with $8 \%$ Ni volume fraction, along with the detail view of peaks at the range of $-1.3 \sim-1.0$, and (c) saturated $\mathrm{Ni}$-doped $\mathrm{W}$ with $75 \% \mathrm{Ni}$ volume fraction, along with the detail view of peaks at the range of $-1.3 \sim-1.0$

Figure 29: Electron density plots corresponding to the density of states as a function of Ni-volume fraction.

Figure 30: Comparisons of the GB strength as a function of volume fraction with analytical result derived from the proposed relation for (a) unsaturated Ni-doped W (GB thickness $=0.3 \mathrm{~nm}$ ), and (b) saturated Ni-doped W (GB thickness $=0.6 \mathrm{~nm})$.

Figure 31: Comparison of the maximum tensile stress with the density of states (sum in the range of $-1.3 \sim-1.0 \mathrm{eV}$ ) for f-orbital of (a) unsaturated case (b) saturated case.

Figure 32: Phonon dispersion of the base $W$ structures, which are cases of no GB (GB thickness $=0 \mathrm{~nm})$, unsaturated $\mathrm{W}(\mathrm{GB}$ thickness $=0.3 \mathrm{~nm})$, and saturated $\mathrm{W}(\mathrm{GB}$ thickness $=0.6 \mathrm{~nm})$.

Figure 33: Phonon dispersion of different interface structure with respect to the direction of (a) $\mathrm{x}$-axis $[\xi 00]$ and (b) diagonal $[\xi \xi 0]$.

Figure 34: Modification of microstructural geometry.

Figure 35: Simple cubic grains.

Figure 36: Crack path of 3D XFEM simulation.

Figure 37: Crack propagation with von Mises stress contour of (a) initial stage, (b) middle stage, and (c) end stage.

Figure 38: Schematic illustration of enriched nodes for crack.

Figure 39: Dimension of the model.

Figure 40: Structural frames of the grains in the model.

Figure 41: Meshed model with each element numbered.

Figure 42: Model with the properties applied to each elements belonging to their phase types.

Figure 43: Plot of the crack propagation during the XFEM simulation.

Figure 44: Crack propagation paths with variation of element size.

Figure 45: Schematic description of proposed method.

Figure 46: Image of polysrystalline tungsten with three selections of typical GB regions.

Figure 47: Schematic illustration of boundary conditions and traction applied.

Figure 48: Three-dimensional models of GB region and the crack propagation samples.

Figure 49: Illustration of boundary condition with crack. 
Figure 50: Fracture paths in W-Ni-Fe.

Figure 51: Crack propagation paths in microstructures with (a) single initial crack, (b) a void existing in arbitrary location, and (c) initial crack and a void existing in single model using XFEM technique.

Figure 52: Digitalization process of microstructural morphology with (a) original image of polycrystalline tungsten, (b) extraction of edges of GBs, and (c) completion of digitalization process by applying grain types into grains.

Figure 53: Cohesive energy dissipation plots for the three cases of simulation which have (a) high fracture strength GBs, (b) medium fracture strength GBs, and (c) low fracture strength GBs at $\mathrm{t}=155$ nanosecond (ICOHE is the cohesive energy dissipation that was scaled down to have range of 0 to 1 ).

Figure 54: Damage plots of three different cases of simulation which include (a) high fracture strength GBs, (b) medium fracture strength GBs, and (c) low fracture strength GBs at $\mathrm{t}=155$ nanosecond (IFAIL is the Damage variable with range of 0 to 1 ).

Figure 55: Plot of cohesive energy as a function of crack length for (a) morphology 1 and (b) morphology 2.

Figure 56: (a) Plot of crack length as a function of time for grains and interface where solid line indicates grains and dotted line indicates interface, (b) plot of cohesive energy dissipation as a function of time.

Figure 57: Percentages of primary crack and microcracks for different cases of simulation which contain (a) high fracture strength GBs, (b) medium fracture strength GBs, and (c) low fracture strength GBs.

Figure 58: Evaluated $M$ values with distributed $m$ values of three cases of study (a) on morphology 1 , and (b) morphology 2.

Figure 59: Comparison of the analytical solution with simulation results for (a) morphology 1 and (b) morphology 2 .

Figure 60: Schematic illustration simulation setup and boundary condition for the cohesive finite element method.

Figure 61: Schematic illustration of the embedded crack with plastic zone.

Figure 62: Damage as a function of energy dissipation for different element size and maximum element size in convergence study.

Table I: Regular solution parameters ( $\Omega$ 's) from the SGTE binary database.

Table II: Regular solution parameters ( $\Omega$ 's) for chosen dopants from the SGTE binary database.

Table III: Raw Materials (All were purchased from Alfa Aesar except that PMMA was purchased from Lucite International and SA was purchased from Fisher Chemicals) 


\section{References}

1 J. Luo, Curr Opin Solid St M 12, 81 (2008).

$2 \quad$ J. Luo and X. M. Shi, Appl Phys Lett 92 (2008).

3 V. K. Gupta, D. H. Yoon, H. M. Meyer, and J. Luo, Acta Mater 55, 3131 (2007).

4 J. Luo, V. K. Gupta, D. H. Yoon, and H. M. Meyer, Appl Phys Lett 87 (2005).

$5 \quad$ X. M. Shi and J. Luo, Appl Phys Lett 94 (2009).

$6 \quad$ X. Shi and J. Luo, Phys Rev Lett 105, 236102 (2010).

$7 \quad$ P. Gustafson, Calphad 12, 227 (1988).

$8 \quad$ X. Shi and J. Luo, Phys Rev B 84, 014105 (2011).

$9 \quad$ P. Wynblatt, A. Saúl, and D. Chatain, Acta Mater 46, 2337 (1998).

10 I. J. Toth, N. A. Lockington, and L. W. Derry, Journal of the Less Common Metals 9, 157 (1965).

11 T. G. Nieh, Scripta Metallurgica 18, 1279 (1984).

12 N. M. Hwang, Y. J. Park, D. Y. Kim, and D. Y. Yoon, Scripta Mater 42, 421 (2000).

13 Y. Mishin, W. J. Boettinger, J. A. Warren, and G. B. McFadden, Acta Mater 57, 3771 (2009).

14 E. N. Hodkin, M. G. Nicholas, and D. M. Poole, Journal of the Less Common Metals 20, 93 (1970).

15 C. Bungaro, K. M. Rabe, and A. D. Corso, Phys Rev B 68, 134104 (2003).

16 N. Moës, J. Dolbow, and T. Belytschko, International Journal for Numerical Methods in Engineering 46, 131 (1999).

17 H. Lee and V. Tomar, Journal of Engineering Materials and Technology 133, 041001 (2011).

18 N. Sukumar, N. Moës, B. Moran, and T. Belytschko, International Journal for Numerical Methods in Engineering 48, 1549 (2000).

19 T. Rabczuk, P. M. A. Areias, and T. Belytschko, International Journal for Numerical Methods in Engineering 72, 524 (2007). 
20 R. W. Rice and S. W. Freiman, J Am Ceram Soc 64, 350 (1981).

21 R. W. Margevicius, J. Riedle, and P. Gumbsch, Materials Science and Engineering: A 270, 197 (1999).

22 R. W. Klopp and D. A. Shockey, J Appl Phys 70, 7318 (1991).

23 G. R. Johnson, R. A. Stryk, S. R. Beissel, and T. J. Holmquist, International Journal of Impact Engineering 27, 997 (2002).

24 A. Loubens, C. Rivero, P. Boivin, B. Charlet, R. Fortunier, and O. Thomas, MRS Proceedings 875 (Investigation of local stress fields: Finite element modelling and High Resolution X-Ray Diffraction).

25 F. Erdogan and G. C. Sih, Journal of Basic Engineering 85, 519 (1963).

26 G. C. Sih and B. Macdonald, Engineering Fracture Mechanics 6, 361 (1974).

27 R. J. Nuismer Jr and J. D. Achenbach, Journal of the Mechanics and Physics of Solids 20, 203 (1972).

28 J. Zhai, V. Tomar, and M. Zhou, Transactions-American Society of Mechanical Engineers Journal of Engineering Materials and Technology 126, 179 (2004). 


\section{List of Acronyms and Abbreviations}
AES:
Auger Electron Spectroscopy
CALPHAD: $\quad$ Calculation of Phase Diagram
CFEM: $\quad$ Cohesive Finite Element Method
CPMD: $\quad$ Car-Parrinello Molecular Dynamics
DOS: $\quad$ Density of States
GB: $\quad$ Grain Boundary
HRTEM: $\quad$ High-Resolution Transmission Electron Microscopy
MD: $\quad$ Molecular Dynamics
NWPW: $\quad$ NWChem Plane-Wave
XFEM: $\quad$ Extended Finite Element Method 


\section{Appendices}

\section{(A) Personnel}

- Dr. Jian Luo, a Professor at Clemson University and UCSD, was the original PI for this project for 07/01/2010 - 12/31/2013 and a UCSD subcontractor for 01/01/2013 06/30/2013; Dr. Luo led the efforts on thermodynamic theories/modeling and experimental validation of selected systems

- Dr. Vikas Tomar, an Associate Professor at Purdue University, was a co-PI for this project; Dr. Tomar led the efforts on multiscale modeling.

- Dr. Fei Peng, Assistant Professor at Clemson University, was designated as the "managing" PI for this project at Clemson University for 01/01/2013 - 06/30/2013 (since Dr. Luo transferred to UCSD on January 1, 2013).

- Naixie Zhou, a Ph.D. student at Clemson University and UCSD, conducted thermodynamic modeling.

- Zhao Zhang, a graduate student at Clemson (supported as a Teaching Assistantship \& received no support from this NETL project) and Mojtaba Samiee, a Ph.D. student at UCSD (partial/minor support in 2013), conducted the experiments for this project.

- Hongsuk Lee, a Ph.D. student at Purdue University, conducted multiscale modeling.

\section{(B) Cost Status}

- Total Federal Funds Awarded: $\$ 300,000$

- Total Federal Funds Spent: $\$ 300,000$

- Total Non-Federal Funds (Clemson Cost Share): \$28,196

- Unspent Funds at the End of This Project: $\$ 0.00$ 


\section{(C) Milestone Status}

\begin{tabular}{|c|c|c|c|c|c|c|c|c|c|c|c|c|c|c|c|c|c|c|}
\hline \multirow{3}{*}{ Milestones } & \multirow{3}{*}{ Milestone Description } & \multicolumn{12}{|c|}{ Project Duration Start: July 1, 2010 End: June 30, 2013} & \multirow{3}{*}{$\begin{array}{c}\text { Planned } \\
\text { Start } \\
\text { Date }\end{array}$} & \multirow{3}{*}{$\begin{array}{l}\text { Planned } \\
\text { End } \\
\text { Date }\end{array}$} & \multirow{3}{*}{$\begin{array}{c}\text { Actual } \\
\text { Start Date }\end{array}$} & \multirow{3}{*}{$\begin{array}{l}\text { Actual } \\
\text { End } \\
\text { Date }\end{array}$} & \multirow{3}{*}{ Comments } \\
\hline & & \multicolumn{4}{|c|}{ Project year (PY) 1} & \multicolumn{4}{|c|}{ PY 2} & \multicolumn{4}{|c|}{ PY 3} & & & & & \\
\hline & & Q1 & Q2 & Q3 & Q4 & Q5 & Q6 & Q7 & $\mathrm{Q} 8$ & Q9 & Q10 & Q11 & Q12 & & & & & \\
\hline Milestone 1 & $\begin{array}{c}\text { Develop a } \\
\text { thermodynamic } \\
\text { description for high-T } \\
\text { premelting-like GB } \\
\text { segregation in } \\
\text { multicomponent } \\
\text { refractory alloys. }\end{array}$ & & & & & & & & & & & & & $\begin{array}{c}\text { July 1, } \\
2010\end{array}$ & $\begin{array}{c}\text { Sept 30, } \\
2011\end{array}$ & $\begin{array}{l}\text { July 1, } \\
2010\end{array}$ & $\begin{array}{c}\text { Sept 30, } \\
2011\end{array}$ & $\begin{array}{l}\text { Completed as } \\
\text { planned }\end{array}$ \\
\hline Milestone 2 & $\begin{array}{l}\text { Develop multiscale } \\
\text { modeling strategies } \\
\text { and validate the } \\
\text { theories and modeling } \\
\text { results on selected } \\
\text { material systems. }\end{array}$ & & & & & & & & & & & & & $\begin{array}{c}\text { July 1, } \\
2010\end{array}$ & $\begin{array}{c}\text { June 30, } \\
2013\end{array}$ & $\begin{array}{c}\text { July 1, } \\
2010\end{array}$ & $\begin{array}{c}\text { June } 30 \\
2013\end{array}$ & $\begin{array}{l}\text { Completed as } \\
\text { planned }\end{array}$ \\
\hline
\end{tabular}

\section{(D) List of Refereed Journal Publications Acknowledged This Grant}

- H. Lee and V. Tomar, "An examination of nickel doping effect on the mechanical strength of a tungsten grain boundary" Computational Materials Science, 77: 131-138 (2013)

- H. Lee and V. Tomar, "Understanding effect of grain boundaries in the fracture behavior of polycrystalline tungsten under mode-I loading," Journal of Engineering Materials and Technology - Transaction of ASME 134: 031010 (2013)

- J. Luo, "Developing Interfacial Phase Diagrams for Applications in Activated Sintering and Beyond: Current Status and Future Directions" (review article), Journal of the American Ceramic Society, 95, 2358 (2012)

- H. Lee and V. Tomar, "Effect of Meso to Micro Transition in Morphology Dependent Fracture of SiC Ceramics," ASME-J Engineering Materials and Technology, 133: 041001 (10 pp) (2011).

- X. Shi \& J. Luo, "Decreasing the Grain Boundary Diffusivity in Binary Alloys with Increasing Temperature," Physical Review Letters, 105, 236102 (2010) 